Universidad Politécnica de Madrid

Escuela Técnica Superior de Ingenieros Navales

Departamento de Arquitectura, Construcción y Sistemas Oceánicos y Navales

\title{
On the analysis of the scale effects in marine propellers using computational tools.
}

\author{
Amadeo Morán Guerrero \\ M.Sc. in Naval Architecture
}

Ph.D. Thesis

Supervisor: Prof. Leo de Miguel González Gutiérrez 

To my family, to the heads of it. 


\section{Abstract}

Nowadays one of the most accurate, reliable and globally accepted methods to estimate the torque and thrust values of a particular propeller from model test results, is the open water test. Subsequently, these results should be corrected with an extrapolation model due to the inability to achieve the Reynolds similarity between the model and real scale conditions. There is still a lot of controversy about the extrapolation models to use and whether the world-accepted models are valid for modern propeller designs. Thanks to the improvements of the mathematical models for fluid dynamic simulations and the increase of computational power, nowadays, when the main objective is to compare different alternatives, more and more numerical simulations are performed for the open water test at a relatively low cost. In this thesis, a study of the most appropriate models to simulate model scale propellers, including the complexity associated to laminar to turbulent transition, is accomplished for several propellers. Thanks to the inclusion of these models it has been possible to analyse the drag and lift scale effects for a particular propeller, being the lift scale effects an important factor of the scale effects for this particular case. Finally, two new models to estimate the friction coefficient of the sections of the propeller are presented, one of them being a modification of a current method closer to $C F D$ results and the other a neural networks application to this particular problem. To carry out both methods a dataset of seven propellers has been used.

\section{Keywords}

CFD; marine propellers; RANS; transition modeling; cross-flow transition mechanism; scaling effects; lift scale effects; open water simulations; extrapolation methods; scaling procedures. 


\section{Resumen}

Actualmente una de las formas más precisas, fiables y aceptadas a nivel mundial para conocer los valores de par y empuje de un propulsor determinado es a partir de ensayos a escala modelo, concretamente con el ensayo de propulsor aislado. Debido a la imposibilidad de cumplir la igualdad de Reynolds entre los ensayos y la escala real es necesario pasar posteriormente estos resultados por el filtro de los modelos de extrapolación, a través de los cuales se le aplican las correcciones a los resultados obtenidos. Todavía hay bastante controversia acerca de los modelos de extrapolación a utilizar y de si dichos modelos, mundialmente aceptados, son válidos para los diseños actuales de hélices. Gracias a los avances en los modelos matemáticos para simulación de fluidos y a las mejoras en potencia de cálculo, hoy en día, cuando se trata de comparar distintas alternativas, cada vez más se opta por realizar simulaciones numéricas del ensayo de propulsor aislado a un coste relativamente bajo. En esta tesis, se estudian mediante herramientas computacionales los modelos más apropiados para la simulación de propulsores a escala modelo, incluyendo la modelización del fenómeno de la transición de flujo laminar a turbulento. Gracias a la inclusión de esos modelos se ha podido realizar un estudio de los efectos de escala debidos a la variación de la resistencia y la sustentación de las secciones para un propulsor, siendo esta última un factor importante en los efectos de escala en este caso. Finalmente, se presentan dos nuevos modelos alternativos para la obtención de la resistencia de fricción de las secciones del propulsor, uno de ellos realizando una pequeña modificación de uno ya existente y otro aplicando redes neuronales a este problema en concreto. Para este último estudio se ha utilizado una base de datos de siete propulsores.

\section{Palabras Clave}

CFD; hélices marinas; RANS; modelos de transición; transición inducida por flujo transversal; efectos de escala; efectos de escala debidos a la sustentación; simulación de curvas de propulsor aislado; métodos de extrapolación. 


\section{Acknowledgments}

I would like to say thanks to Juan González Adalid and Mariano Pérez Sobrino, it seems that it was yesterday when we started working together, a lot of things have happened but it has undoubtedly been a nice stage of the road. I have learned too many things in our meetings, discussions and meals.

Thanks to my Director, Dr. Leo Miguel González Gutiérrez for his time and support during this years. Sometimes we agree, sometimes not, always interesting discussions in any case. Thanks for the possibility to see the academic world from inside, I really appreciate the projects that we have been working together before and during this thesis, these have contributed to the completion of this work.

Special thanks to SISTEMAR S.A. and Universidad Politécnica de Madrid as co-financers of this thesis.

I would like to make a general thanks to the people of the European Union and particularly the Spaniards. Thanks to their taxes it is possible to maintain the public system that have permitted me to carry out part of this work and in the projects that I have worked on.

Particularly thanks to my friend Héctor, to show me the Canary pace during ours walkarounds.

And finally thanks to the most special person in my life and the future mother of my childrens, for the last Christmas and weeks without boring house cleaning and food preparations until the end of this thesis, and for her continuous support and determination to really make possible to conclude this work. 


\section{Contents}

1 Introduction 1

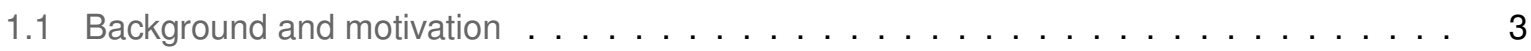

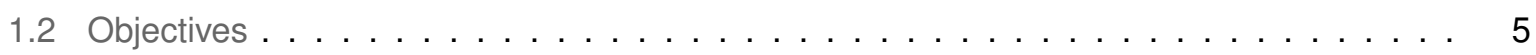

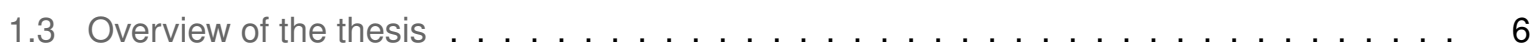

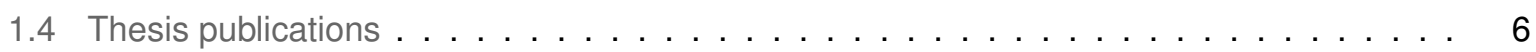

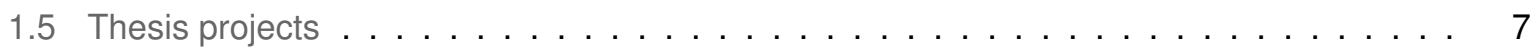

2 General considerations $\quad 9$

2.1 Scaling problem on propellers $\ldots \ldots \ldots \ldots \ldots \ldots \ldots \ldots \ldots$

2.1 .1 Statistical methods . . . . . . . . . . . . . . . . . . . . 11

2.1 .2 Analytical methods . . . . . . . . . . . . . . . . . . . . . . 12

2.1 .3 Full-Scale CFD simulations . . . . . . . . . . . . . . . . . . . . 13

2.2 Numerical approaches to model laminar-turbulent transition . . . . . . . . . . . . . 13

2.2.1 Brief description of the $\gamma-R e_{\theta}$ transition model . . . . . . . . . . . . . . 14

2.3 Artificial Neural Networks applied to the scaling problem on marine propellers $\ldots \ldots \ldots$

2.3.1 Friction coefficient prediction $\ldots \ldots \ldots \ldots \ldots \ldots$

2.3.2 Direct $K_{t}$ and $K_{q}$ extrapolation ratio $\ldots \ldots \ldots \ldots \ldots \ldots \ldots \ldots$

3 Results 21

3.1 Cross-flow specific study: M2355 profile . . . . . . . . . . . . . . . . . 23

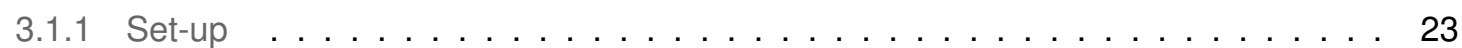

3.1 .2 Results . . . . . . . . . . . . . . . . . . . . 25

3.2 Different turbulent approaches comparison at model scale propellers simulations . . . . 28

3.2.1 P1727 Tip-Rake Propeller . . . . . . . . . . . . . . . . . . . . . . . . . . . . . 29

3.2.2 New generation of $\mathrm{CLT} \AA \mathrm{B}$ Propeller . . . . . . . . . . . . . . . . . . . . 34

3.2 .3 VP1304 conventional Propeller . . . . . . . . . . . . . . . . . . . . 37

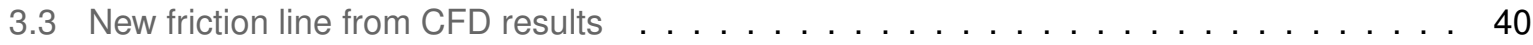

3.4 New Form factor and lift scale effects $\ldots \ldots \ldots \ldots \ldots \ldots \ldots$

3.4.1 Identification of the AoA of the sections, $\alpha_{\beta_{i}} \ldots \ldots \ldots \ldots \ldots$ 
3.4 .2 Form factor modification . . . . . . . . . . . . . . . . 50

3.4 .3 Lift scale effects . . . . . . . . . . . . . . . . . . . . 51

3.5 Analysis of different extrapolation methods. . . . . . . . . . . . . . 54

3.5.1 Evaluation of the improvement with the new form factor $k_{M L P}$ and lift scale effects

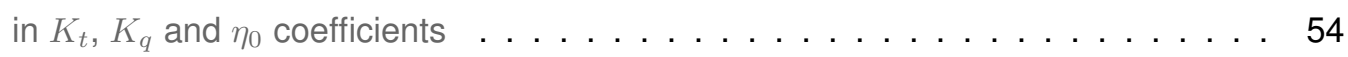

3.5.2 Comparison of different extrapolation methods with and without lift scale effects. . 56

4 Conclusions $\quad 59$

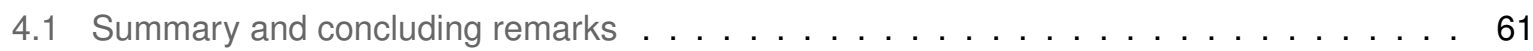

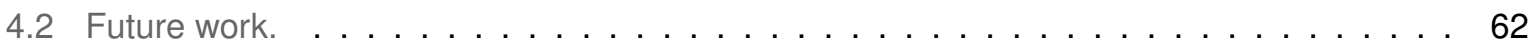

$\begin{array}{ll}\text { A Geometries } & 69\end{array}$

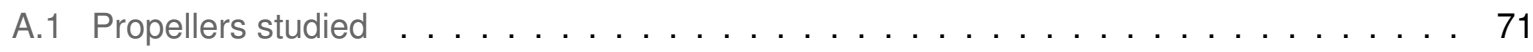

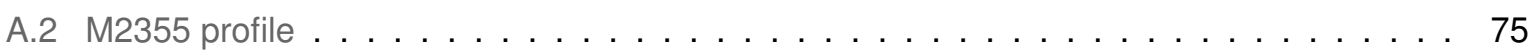

$\begin{array}{ll}\text { B Contour plot and sectional data distributions } & \mathbf{7 6}\end{array}$

B.1 VP1304. . . . . . . . . . . . . . . . . . . . . . . 77

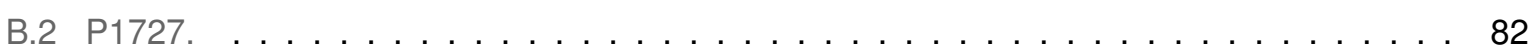

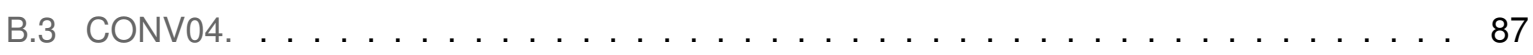

C Mesh convergence for section 3.2.

D Detailed description of the new friction coefficient estimation methods 98

D.1 Modified STRIPSIS method. . . . . . . . . . . . . . . . . . . . . . 99

D.2 Multilayer Perceptron Friction coefficient estimator. . . . . . . . . . . . . . . 100 


\section{List of Figures}

1.1 Boundary layer scheme where crossflow effects are included from ref: (a) Saric et al. (2003) and a (b) boundary layer extracted from CFD computation. . . . . . . . . . . 5

$2.1 \quad M L P$ basic scheme. . . . . . . . . . . . . . . . . . . . . . 17

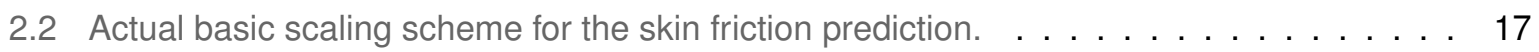

2.3 Proposed scaling scheme for the skin friction prediction with the usage of $M L P \ldots \ldots$

2.4 Actual basic scaling scheme for the direct scaling of $K_{t}$ and $K_{q} \ldots \ldots \ldots \ldots \ldots$

2.5 Proposed scheme of the $M L P$ for the direct estimation of the scaling factors $R_{K t}$ and $R_{K q} .19$

3.1 Mesh convergence test where the transition onset criteria is monitored on suction and pressure sides. . . . . . . . . . . . . . . . . . . . . . . . . . 24

3.2 Transition onset on suction side for different $A o A \ldots \ldots \ldots \ldots \ldots \ldots \ldots$

3.3 Transition onset on pressure side for different $A \circ A \ldots \ldots \ldots \ldots \ldots$

3.4 Transition onset in suction side (top) and pressure side (bottom) for different $A o A$ and different values of the turbulence intensity. . . . . . . . . . . . . . . 27

3.5 Geometries of the three propellers studied: VP1304 (a), P1727 (b) and the new generation of CLT propeller (c) . . . . . . . . . . . . . . . . . . . . 28

3.6 Differences on the efficiency values with respect to the experimental values for the P1727 propeller. . . . . . . . . . . . . . . . . . . . 30

3.7 Differences in dimensionless thrust and torque coefficients with respect to the experimental values for the $\mathrm{P} 1727$ propeller. . . . . . . . . . . . . . . . . . . . . 31

3.8 Scheme of the different sections used for the local transition study $\ldots \ldots \ldots \ldots \ldots \ldots$

3.9 Skin friction comparison for different dimensionless radius for P1727 ITTC benchmark

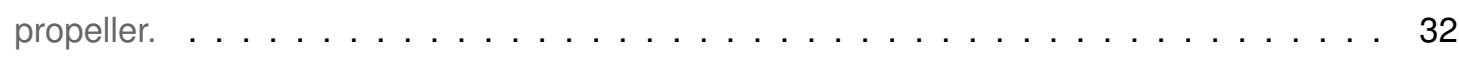

3.10 Intermittency contour plot at $r / R=0.9375$ of the non-conventional P1727 Tip-Rake Pro-

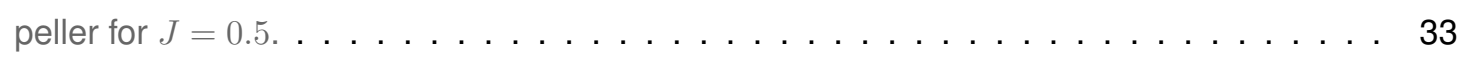


3.11 Skin friction contour plot and streamlines representation over the suction (top) and pressure (bottom) sides for the P1727 ITTC Benchmark propeller at $J=0.5 \ldots \ldots \ldots$. . . . . 33

3.12 Efficiency values of the open water test for the CLT propeller. . . . . . . . . . . . . . 35

3.13 Differences in dimensionless thrust and torque respect to the experimental values for the CLT propeller. . . . . . . . . . . . . . . . . . . . . . . . . .

3.14 Skin friction comparison for different dimensionless radius for CLT propeller at design

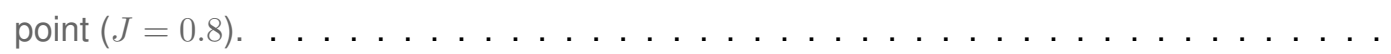

3.15 Intermittency scalar value for a cylindrical section of the CLT propeller at dimensionless radius $r / R=0.81$ and $J=0.8 \ldots \ldots \ldots \ldots \ldots \ldots \ldots$

3.16 Skin friction contour plot and streamlines representation over the suction (top) and pressure (bottom) sides for the CLT propeller at $J=0.8 \ldots \ldots \ldots \ldots \ldots$. . . . . . 37

3.17 Efficiency values of the open water test for the VP1304 propeller. . . . . . . . . . . .

3.18 Differences in dimensionless thrust and torque with respect to the experimental values for the VP1304 propeller. . . . . . . . . . . . . . . . .

3.19 Skin friction comparison for different dimensionless radii for the VP1304 ITTC benchmark

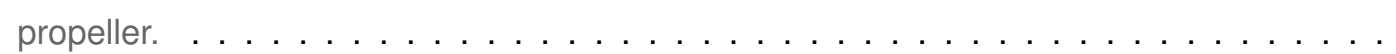

3.20 Intermittency scalar value for a cylindrical section of the VP1304 ITTC benchmark propeller at dimensionless radii $r / R=0.9375$ and $J=1.2 \ldots \ldots \ldots \ldots$

3.21 Skin friction contour plot and streamlines representation over the suction (top) and pressure (bottom) sides for the VP1304 ITTC Benchmark propeller at $J=1.2 \ldots \ldots \ldots \ldots$. . 41

$3.22 C_{f}$ for all the sections studied separated in pressure and suction side. . . . . . . . . . 42

3.23 Dimensionless wall shear stress for all the propeller studied. . . . . . . . . . . . . . 43

$3.24 C_{f}$ for each section studied compared with the most common friction lines. . . . . . . 44

3.25 $C_{f}$ for each section studied compared with the two new methods presented, STRIPSIS $S_{\text {modified }}$ and $M L P$. Blasius and Prandt-Von Karman lines are reference lines. . . . . . . . . . 45

3.26 Difference between the full and model scale friction coefficients $\Delta C_{f}$ for all the methods presented at this section. . . . . . . . . . . . . . . . .

3.27 Mean and standard deviation of the difference between the $\Delta C_{f}$ for all the points of of each method $\left(\Delta C_{f}^{\text {method,i }}\right)$ and $\Delta C_{f}$ obtained with $C F D$ results. . . . . . . 46

3.28 Marine propeller blade profile forces and velocities scheme. . . . . . . . . . . . . 48

3.29 Lift coefficient for different projection angles. The angle that satisfies $\max \left(C_{\text {Lift }}\right)$ is represented by a red line. Full scale simulation - radius $0.35 \mathrm{R}-5 \mathrm{rps} . \ldots \ldots \ldots$

3.30 Lift coefficient for different projection angles. The angle that satisfies $\max \left(C_{\text {Lift }}\right)$ is represented by a red line. Model scale simulation - radius $0.35 \mathrm{R}-5 \mathrm{rps} \ldots \ldots \ldots \ldots$. . . 
3.31 Angle of the incident flow $\alpha_{\beta_{i}}$ along the radii for different Reynolds of the $O W T$ simulations. Results for different rotating velocities at model scale and full scale are represented.

3.32 CONV02 sections comparison for different ways to estimate the $C_{D}$. The case represented is the $O W T$ simulation for model scale at $r p s=5$. Blasius and Prandtl lines are plotted only as reference values. . . . . . . . . . . . . . . . . . .

3.33 CONV02 propeller $C_{D}^{\text {diff }}(\%)$ mean values for all the range studied, using different form

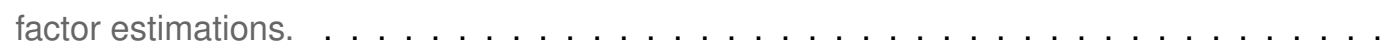

3.34 CONV02 propeller $\Delta K_{t}$ and $\Delta K_{q}$ due to the lift scale effects, along the radii for the different rps studied. . . . . . . . . . . . . . . . . . . . .

3.35 CONV02 propeller $\Delta K_{t}$ and $\Delta K_{q}$ due to the lift scale effects, along the radii for the different rps studied. . . . . . . . . . . . . . . . . . . . . . .

3.36 $\Delta K_{t}$ and $\Delta K_{q}$ comparison for the extrapolated values using the $k_{I T T C 78}$, the $k_{M L P}$, the $k_{M L P}$ plus lift corrections and the direct CFD simulations for CONV02 propeller. . . . . .

3.37 CONV02 propeller $\eta_{0}$ comparison for the extrapolated values using the $k_{I T T C 78}$, the $k_{M L P}$, the $k_{M L P}$ plus lift corrections and direct $C F D$ simulations. . . . . . . . . .

3.38 CONV02 propeller $\Delta K_{t}$ and $\Delta K_{q}$ comparison for different extrapolation methods without applying lift corrections, and direct $C F D$ simulations. . . . . . . . . . . . .

3.39 CONV02 propeller $\Delta K_{t}$ and $\Delta K_{q}$ comparison for different extrapolation methods applying lift corrections calculated in section 3.4, and direct $C F D$ simulations. . . . . . . . . . .

3.40 Bar diagram representing the results of the Equation 3.12 with and without lift scale effects corrections. . . . . . . . . . . . . . . . . . . . . . .

3.41 Bar diagram representing the results of the Equation 3.11 with and without lift scale effects corrections. . . . . . . . . . . . . . . . . . . . . .

A.1 Frontal view for all the propellers studied. $\ldots \ldots \ldots \ldots \ldots \ldots$

B.1 Skin friction contour plot and streamlines representation over the suction (top) and pressure (bottom) sides for the VP1304 propeller at $J=1.0$ for different Reynolds numbers. . 77

B.2 Suction side of the $C_{p}$, for different radii of the VP1304 propeller. $\ldots \ldots \ldots \ldots \ldots$

B.3 Pressure side of the $C_{p}$, for different radii of the VP1304 propeller. . . . . . . . . . 79

B.4 Suction side of the $C_{f, n t}$, for different radii of the VP1304 propeller. . . . . . . . . . 80

B.5 Pressure side of the $C_{f, n t}$, for different radii of the VP1304 propeller. . . . . . . . . 81

B.6 Skin friction contour plot and streamlines representation over the suction (top) and pressure (bottom) sides for the P1727 propeller at $J=0.565$ for different Reynolds numbers. . 82

B.7 Suction side of the $C_{p}$, for different radii of the $\mathrm{P} 1727$ propeller. . . . . . . . . . 83

B.8 Pressure side of the $C_{p}$, for different radii of the $\mathrm{P} 1727$ propeller. . . . . . . . . . . 84 


\section{LIST OF FIGURES}

B.9 Suction side of the $C_{f, n t}$, for different radii of the $\mathrm{P} 1727$ propeller. . . . . . . . . . 85

B.10 Pressure side of the $C_{f, n t}$, for different radii of the P1727 propeller. . . . . . . . . . 86

B.11 Skin friction contour plot and streamlines representation over the suction (top) and pres-

sure (bottom) sides for the CONV04 propeller at $J=0.8105$ for different Reynolds numbers. 87

B.12 Suction side of the $C_{p}$, for different radii of the CONV04 propeller. . . . . . . . . 88

B.13 Pressure side of the $C_{p}$, for different radii of the CONV04 propeller. . . . . . . . . . 89

B.14 Suction side of the $C_{f, n t}$, for different radii of the CONV04 propeller. . . . . . . . . . 90

B.15 Pressure side of the $C_{f, n t}$, for different radii of the CONV04 propeller. . . . . . . . 91

C.1 Scheme of the parameters used for the convergence process in the boundary layer mesh. 93

C.2 Left: Mesh view close to the propeller. Right: Detailed view of the mesh in the leading edge proximities. . . . . . . . . . . . . . . . . . . . . . . . . . . . 94

C.3 Mesh convergence process for VP1304 propeller based on the $K t$ and $10 K q$ non-dimensional parameters for different number of prism layers. Dotted grey lines represent the convergence process when the global mesh size is fixed and the number of prism layers is increased. . . . . . . . . . . . . . . . . . . . . . . . 95

C.4 Mesh Convergence process for the P1727 propeller based on the Kt and $10 K q$ nondimensional parameters for different number of prism layers. Dotted grey lines represent the convergence process when the global mesh size is fixed and the number of prism layers is increased. . . . . . . . . . . . . . . . . .

C.5 Mesh Convergence process for different numbers of layers in the boundary layer for CLT propeller. Kt and Kq values. . . . . . . . . . . . . . . . . . . . . .

D.1 Estimation of the friction coefficients for the Original ST RIPSIS and modified STRIPSIS methods for differents sections of the blade. . . . . . . . . . . . . . . . . . . . 99

D.2 Base $A N N$ scheme. . . . . . . . . . . . . . . . . . . . . . . . . . 101

D.3 Mean loss of the 20 last iteration of the different cases studied for select the optimum one MLP configuration. . . . . . . . . . . . . . . . . . . . . . 102

D.4 Residuals comparison of the final MLP configuration and one of the alternatives studied. 102

D.5 Residuals comparison for different activation function of the hidden layers. . . . . . . . 103 


\section{List of Tables}

3.1 Main parameters of the M2355 profile simulation. . . . . . . . . . . . . . . . 24

3.2 Main geometric characteristics of the ship propellers studied. . . . . . . . . . . . . . . . 29

3.3 General parameters for the different propellers. Reynolds are calculated at $J=1.0$ for the VP1304, $J=0.5$ for the P1727 and $J=0.8$ for the CLT. . . . . . . . . . . . . 29

3.4 Differences on the efficiency values with respect to the experimental values for the P1727

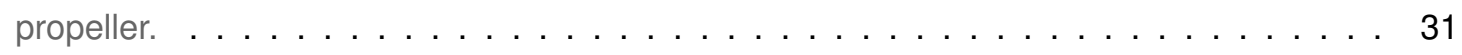

3.5 Open Water results for new generation of CLT propeller with respect to the experimental values. . . . . . . . . . . . . . . . . . . . . . . . . . . . 34

3.6 Differences on the efficiency values respect to the experimental values for the VP1304 propeller. . . . . . . . . . . . . . . . . . . . . . . 38

A.1 Main geometric characteristics of the propellers used for the ITTC benchmark test case. . 72

A.2 Main geometric characteristics of CLT propeller used in this thesis. . . . . . . . . . 73

A.3 Rest of the conventional propellers used in this thesis. . . . . . . . . . . . . . . 74

A.4 Main parameters of the M2355 profile. . . . . . . . . . . . . . . . . . . . 75 


\section{Acronyms}

ANN Artificial Neural Network.

AoA Angle of Attack.

CFD Computational Fluids Dynamics.

CLT Contracted and Loaded Tip Propeller.

CPP Controllable Pitch Propeller.

FPP Fixed Pitch Propeller.

MLP Multilayer Perceptron.

NN Neural Network

OWC Open Water Characteristics.

OWT Open Water Test.

RANS Reynolds Average Navier Stokes.

Re Reynolds number. 
WSST Dimensionless Wall Shear Stress (Chord direction component). 


\section{Nomenclature}

$C_{D, C_{f}}^{C F D} \quad$ Drag coefficient obtained directly from $C F D$.

$C_{D} \quad$ Dimensionless Drag coefficient.

$C_{D}^{C F D} \quad$ Drag coefficient obtained directly from $C F D$.

$C_{L} \quad$ Dimensionless Lift coefficient.

$C_{f, n t} \quad$ Dimensionless Wall Shear Stress (Nose-tail component).

$C_{f} \quad$ Dimensionless Wall Shear Stress.

$C_{p} \quad$ Dimensionless pressure coefficient.

FCR Camber-Chord ratio.

$J \quad$ Advance ratio.

$K_{q} \quad$ Dimensionless Torque.

$K_{t} \quad$ Dimensionless Thrust.

THCR Thickness-Chord ratio.

X0 Dimensionless radius.

$\Delta C_{D} \quad$ Dimensionless Drag coefficient increment between model and full scale, $C_{D}^{s}-C_{D}^{m}$.

$\Delta C_{f} \quad$ Dimensionless friction coefficient increment between model and full scale, $C_{D}^{s}-C_{D}^{m}$.

$\Delta K_{q} \quad$ Dimensionless Torque coefficient increment between model and full scale, $K_{q}^{s}-K_{q}^{m}$.

$\Delta K_{t} \quad$ Dimensionless Thrust coefficient increment between model and full scale, $K_{t}^{s}-K_{t}^{m}$.

$\alpha_{\beta_{i}} \quad$ Angle of Attack of the profile sections of the propeller taking into account the induced velocities. 


\section{Nomenclature}

$\eta_{0} \quad$ Open Water Efficiency.

$\tau \quad$ Wall Shear Stress.

$\tau_{n t} \quad$ Wall Shear Stress (Nose-tail component).

$\overrightarrow{n t} \quad$ Nose-tail unitary vector.

rps Revolutions per second.

w.r.t. $\quad$ With respect to. 


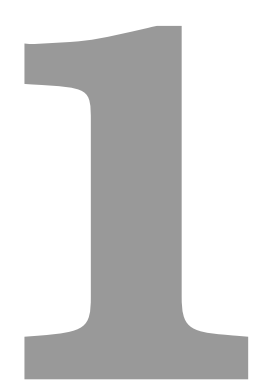

\section{Introduction}

\section{Contents}

1.1 Background and motivation $\ldots \ldots \ldots \ldots \ldots \ldots \ldots \ldots \ldots \ldots$

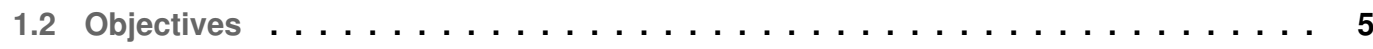

1.3 Overview of the thesis $\ldots \ldots \ldots \ldots \ldots \ldots \ldots \ldots \ldots \ldots \ldots$

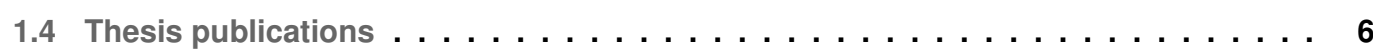

1.5 Thesis projects $\ldots \ldots \ldots \ldots \ldots \ldots \ldots \ldots \ldots \ldots \ldots \ldots$ 
CHAPTER 1. INTRODUCTION 


\subsection{Background and motivation}

Marine propellers are one of the most impressive and mysterious part of the ships. They are the last part responsible to transform the engine energy to ship speed trying to move as less as possible the surrounding flow or, in other words, loosing the minimum energy doing such a thing. The most precise way to know the efficiency of the propeller was to perform an Open Water Test (OWT) in a model basin, accepting the inability to achieve the Reynolds number $(R e)$ similarity. Consequently, it is necessary to make some corrections to the hydrodynamic coefficients of the Open Water Characteristics (OWC), in order to override effects that are not present at full scale due to the different characteristics of the flow over the blade. The methods that apply these corrections are usually known as 'Extrapolation Methods' and the physics behind the corrections are the so-called 'Scale effects'.

Nowadays, computational tools and, in particular, Computational Fluids Dynamics (CFD), are becoming a powerful tool in the engineering process. A few years ago it was impossible to test thousands of geometries in order to select the optimum one or to analyse the sensibility of the design to an specific parameter of the geometry. CFD is able to represent an accurate version of the flow physics, where the main limitation is the computational resources available. Therefore, it would be possible to simulate a propeller at full scale, overcoming the Reynolds similarity. Despite the performance of the mathematical model or the mesh limitations to accurately capture the essence of the phenomena under study, CFD methods have a brilliant present and a astonishing future in the engineering world.

The flow over the propeller at model-scale Reynolds numbers is clearly in the critical Reynolds range, where the flow does not need too many reasons to become turbulent. Flow visualizations at these Reynolds, typically show regions of laminar and turbulent flows over the blade, as for example discussed in Kuiper (1981, 2000); Sánchez-Caja et al. (2014); Bhattacharyya et al. (2016). The paint streak's direction depends much on the shear stress which increases drastically since the laminar to turbulent transition phenomenon occurs. Paint streaks show a higher radial component on laminar regions while they are more azimuthal in the turbulent ones. The laminar part on the propeller blade area is between 50 to $60 \%$ according to Funeno (2002), for this reason marine propellers at model scale are one of those CFD applications where modelling the laminar and turbulent areas are specially important if an accurate prediction and a realistic physical approach is searched. At full scale Reynolds number the flow can be treated as turbulent for two reasons, in the author perspective, due to the Reynolds at which the propeller usually operates that minimizes the laminar part and due to the turbulent characteristic of the incoming flow because of the presence of the hull.

In order to accomplish the purpose of simulating a realistic OWT and analyse the scale effect on 
marine propellers, the use of transition models, to capture laminar to turbulent transition, is necessary. As many authors confirm (e.g. Langtry, 2006), traditional methods for transition prediction based on integral computations are impractical from a computational point of view. This weakness may be overcome with the correlation-based transition models. In this work, the $\gamma-R_{\theta}$ implementation of the StarCCM+ software will be used. This implementation has demonstrated the capacity to deal with flat plates, $2 \mathrm{D}$ airfoils, 3D multi-element airfoils, and even in ship propellers by Bhattacharyya et al. (2015).

The $\gamma-\operatorname{Re} \theta$ transition model is a correlation-based approach for transition modeling that was specifically designed for CFD codes (Menter et al., 2006). The dependance of the model on the correlation is the main drawback of this method, apart of the turbulent inlet quantities that has been studied in the propeller field by Baltazar et al. (2018). Correlation-based transition models using local variables have been in continuous development. In 2004, Menter et al. (2004) first proposed these models as a practical solution to transition prediction in industrial CFD based on complex unstructured meshes (Abu-Ghannam and Shaw, 1980; Menter et al., 2006; Malan et al., 2009). In 2009, Langtry and Menter (2009) presented the full formulation of the $\gamma-R e_{\theta}$ and Malan et al. (2009) presented its implementation in a commercial CFD solver. Nowadays, correlation-based methods for transition prediction are being used in different sectors such as aeronautical or marine industries.

Research studies have identified several types of instabilities such as: attachment line, streamwise, centrifugal and crossflow Saric et al. (2003). Since 1989, flow around rotating disks is presented by Reed and Saric as one of the canonical examples of the crossflow instability in 3D boundary layers. The author considers important to include the crossflow effects as one of the typical causes of laminarturbulent transition at model scale propellers as can be observed in Figure 1.1. Recently, the $\gamma-R e_{\theta}$ transition model was extended to capture crossflow effects by Langtry et al. (2015), without any violation of the LCTM (Local Correlation-based Transition Model) approach. This overcomes one of the weakness of these model, being the correlation method that they previously used only for bypass and natural (TS) transitions.

With the recent developments in the modelization of laminar to turbulent transition in commercial and non-commercial CFD codes, one of the motivation of this thesis was to apply these recent developments to the OWT simulations in marine propellers and try to understand in a better way the phenomena that occur there. With a deeper understanding of those phenomena, it will be possible to create a new extrapolation method for the OWT. 


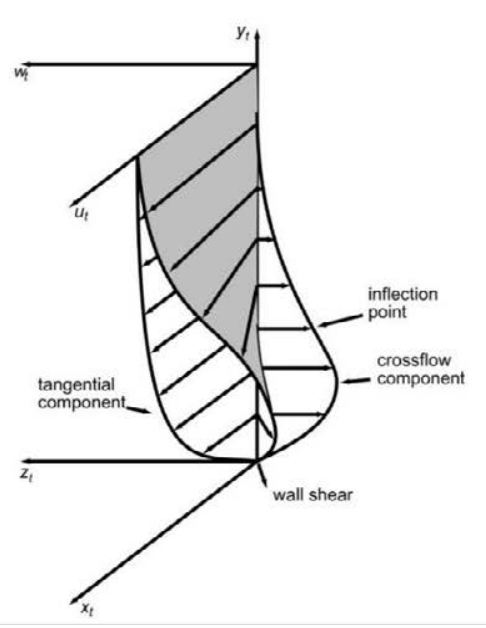

a
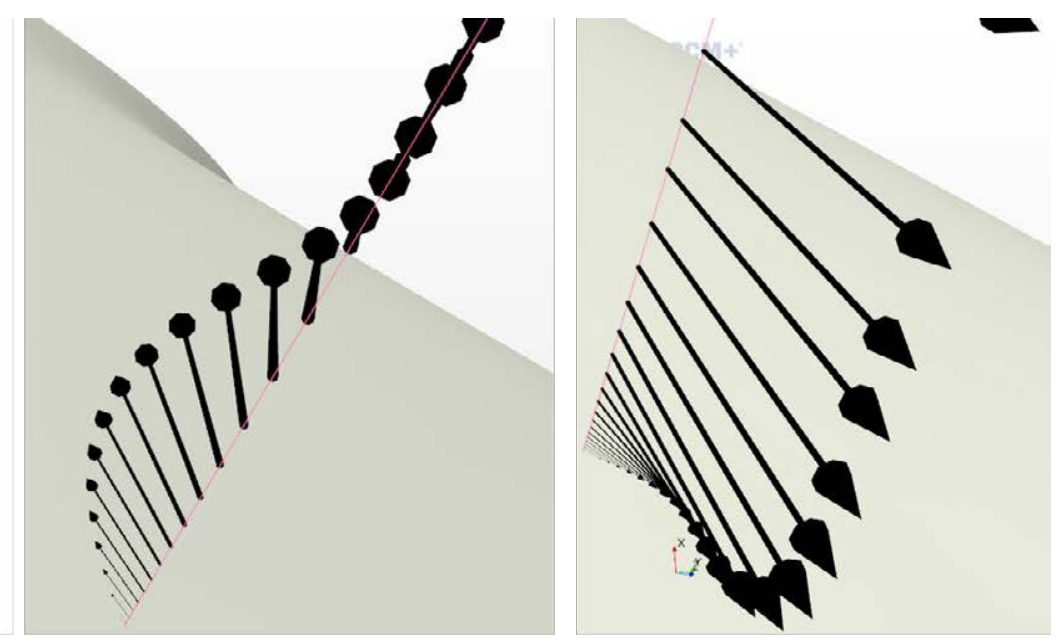

b

Figure 1.1: Boundary layer scheme where crossflow effects are included from ref: (a) Saric et al. (2003) and a (b) boundary layer extracted from CFD computation.

\subsection{Objectives}

The scope of this thesis is to perform an analysis o different turbulence models to use with model scale propellers, including those capable to model transition from laminar to turbulent. This is necessary to perform the second objective of the thesis, evaluate the scale effects for one particular propeller and compare different extrapolation methods with direct CFD simulations. An alternative scope of this thesis is to create a model to estimate the friction coefficient of the propeller sections.

In order to achieve this goal, the following steps have been followed:

- Evaluate the improvements when including the cross-flow transition mechanism in the transition model and compare with experimental tests.

- Analyse the influence of different turbulence models in the open water simulations at model scale, including approaches capable to model transition effects with and without the cross-flow transition mechanism.

- Study the effects of the Reynolds variation in different propellers, taking into account transition effects at model scale to develop a more realistic method to estimate the friction coefficient.

- Analyse the drag coefficient and lift scale effects when full scale and model scale simulations are computed.

- Compare different extrapolation methods and evaluate their performance, comparing with results from CFD simulations at full scale. 


\subsection{Overview of the thesis}

The thesis is organized in four Chapters, including the present one, and four Appendixes.

Chapter 2 introduces general concepts used along the thesis, such as, some of the existing extrapolation methods for scaling OWT, the numerical models used in CFD simulations and the possibilities that artificial neural networks may provide into the extrapolation problem of marine propellers.

Chapter 3 presents the overall results. Different studies are introduced in order to analyse the utility of transition models in CFD and the more specific cross-flow term in the $\gamma-R e_{\theta}$ transition model. Also, two new methods to estimate the friction coefficient of the different sections based on CFD results are presented. To finish this section, an investigation of the form factor and lift scale effect and its influence in different scaling procedures is presented for one particular propeller.

Chapter 4 summarises the main outcomes of the thesis, it presents the concluding remarks and some possible areas for future work.

Appendix A presents the main characteristics of the geometries used along the thesis.

Appendix B shows the complete results extracted from CFD simulations from Section 3.3.

Appendix C details the mesh convergence process for some of the propellers used in Chapter 3.

Appendix D details the creation process of the artificial neural network used in this thesis.

\subsection{Thesis publications}

Partial results of this Thesis have been published in the JCR Journal:

Moran-Guerrero et al. (2018): 'On the influence of transition modelling and crossflow effects on open water propeller simulations.' Ocean Engineering. 156:101 - 119

and presented in the following international conferences:

Moran-Guerrero et al. (2016): 'NNATAC, Preliminary results.' Seventh annual Airbus DiPaRT 2016. Bristol, UK.

Moran-Guerrero et al. (2017): 'Open Water results comparison for three propellers with transition model, applying crossflow effect, and its comparison with experimental results.' Fifth International Symposium on Marine Propulsors, SMP-17. Espoo, Finland.

Collateral results of this Thesis have been presented in: 
Gonzalez-Adalid et al. (2016): 'New Generation of CLTßpropellers.' Maritime Technology and Engineering 3, MARTECH 2016. Lisbon, Portugal.

Perez-Sobrino et al. (2016): 'A new performance prediction procedure for propellers with unconventional tip shape.' 12th International Conference on Hydrodynamics, 18 - 23 September 2016,. Delft, Netherlands.

Gonzalez-Adalid et al. (2018): 'The Use of Modern Computational Tools in the Design Process of Unconventional Propellers for Performance Prediction and Full-Scale Extrapolation.' Proceedings of NAV 2018: 19th International Conference on Ship and Maritime Research. Trieste, Italy.

\subsection{Thesis projects}

This thesis has partially contributed to the development of the following projects:

COINCIDENTE : Optimización de las hélices CLT para operación en buques militares y demostración mediante ensayos del comportamiento en cavitación, presiones inducidas y ruido radiado. Granted by: Spanish Ministry of Defense. Grant: 100321500. Participants: SISTEMAR S.A., UPM.

NNATAC: New Numerical and Analytical Tools for Aerodynamic flow Control. Project Reference: 324298, contract: FP7 People Programme, Granted by: European Union, Participants: UPM, Innovation Works EADS UK Ltd

IMAGE: International Cooperation in Aeronautics with China. H2020 (2014-2020), SC4-Smart, green \& integrated transport, Project reference: 688971, Coordinator: CHALMERS University. 
CHAPTER 1. INTRODUCTION 


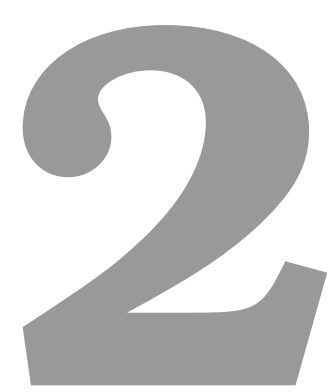

\section{General considerations}

\section{Contents}

2.1 Scaling problem on propellers $\ldots \ldots \ldots \ldots \ldots \ldots \ldots$

2.2 Numerical approaches to model laminar-turbulent transition $\ldots \ldots \ldots$

2.3 Artificial Neural Networks applied to the scaling problem on marine propellers . . 15 
CHAPTER 2. GENERAL CONSIDERATIONS 
In this Section a deeper introduction to the main concepts that are going to be used along this thesis is presented. Firstly, a general overview of the necessity of the extrapolation methods. Second, a more specific explanation of the numerical methods used and finally the possible contribution of the neural networks to the scaling problem on propellers is discussed.

\subsection{Scaling problem on propellers}

Propellers, after the design process, and particularly, when specifically designed for a certain ship, are tested at model scale in a towing tank facility. There are different types of model tests to analyse the behaviour of a certain propeller. In this thesis, we are going to focus in the OWT campaign. This test is carried out to quantify the OWC of the propeller, which are basically the thrust and torque for different advance ratio $J$ values, which are normally adimensionalised and noted in the following as $K_{t}$ and $K_{q}$.

Due to the inability to achieve Reynolds similarity in model test, OWC must be corrected to full scale. There are different methods to apply corrections and consequently obtain the real OWC of the propeller from model test results. A general overview and comparison of the majority of them can be found in Helma et al. (2017). Mostly, from the author's perspective, they can be classified in statistical or analytical based methods (see Sections 2.1.1 and 2.1.2). Also, the OWC can be directly determined performing CFD simulations at full scale (see Section 2.1.3).

\subsubsection{Statistical methods}

These methods are usually based on correlations from sea trials, towing tank test results or, currently, CFD results. Nowadays, the most standard and internationally recognized method for propeller extrapolation is the ITTC - 78 method. This method is based on viscous corrections to the $K_{t}$ and $K_{q}$ coefficients taking into account the Reynolds number and the roughness differences between model and full scale. These corrections are applied to the dimensionless radius 0.75 , considering that the whole blade is well represented by this section (equivalent profile section). This well-known procedure is explained in detail in reference ITTC (2011). However, the ITTC - 78 method is often accused of not meeting today's requirements, especially for modern propeller designs.

Klose et al. (2017) adapted the $I T T C-78$ method for tip raked propellers with a new friction line ${ }^{1}$ for model and full scale, using a more precise version of the form factor for the foil sections and a final

\footnotetext{
${ }^{1}$ Methods to estimate the friction coefficients of the equivalent flat plate are usually known as 'friction lines'.
} 
correction for the boundary layer of the dynamometer.

Another example of these kind of methods is the one presented by Perez-Sobrino et al. (2005) for the Contracted and Loaded Tip Propeller (CLT). The coefficients applied in this last method are extracted from correlations curves between model test and sea trial results. In the case of Perez-Sobrino et al. (2016), the sections of the blade are classified by laminar, transitional or turbulent, depending on certain parameters of the blade sections and flow conditions for both model and full scale Reynolds number. The dimensionless friction forces are calculated differently for each flow region and scale, to finally compute the $\Delta C_{D}$ between both scales for all the sections of the propeller. The increment between model and full scale dimensionless thrust and torque, $\Delta K_{t}$ and $\Delta K_{q}$, are calculated by integration of the $\Delta C_{D}$ along the propeller radii.

It is also important to include the strip method developed by HSVA, TUHH and MMG (Bugalski et al., 2013), being the first one that treated the corrections integrating over the blade. The strip method of HSVA, TUHH and MMG is based on a friction line statistically extracted from extensive CFD simulations using different propellers.

\subsubsection{Analytical methods}

Analytical methods usually apply corrections to the lift and drag coefficients of the propeller blade section $0.75 \mathrm{R}$, being based on the profile knowledge and using, once more, the equivalent profile section. The $C_{L}$ and $C_{D}$ are derived from the OWC.

In the case of Lerbs-Meyne method (see Lerbs, 1951; Meyne, 1968), the calculation of the drag ratio $\left(\epsilon_{0.7}\right)$ of the equivalent profile section at radius $0.7 R$ is performed following an iterative process by comparing the ideal propeller $\left(\eta_{i}\left(\epsilon_{0.7}\right)\right.$ and $\left.C_{T H i}\left(\epsilon_{0.7}\right)\right)$, extracted from the measured values of $\eta$ and $C_{T H}$, and the $\eta_{i}$ and $C_{T H i}$, extracted from the Kramer diagram. The full scale values are calculated with a friction coefficient of 0.006 . This method is valid for propellers that present an optimum circulation distribution, constituting the weakness of the method. As modern propellers are almost fully wake-adapted designs, the optimum circulation distribution is not fulfilled.

Helma (2015) proposed the $\beta_{i}$-method. The original part of this method is to decompose the lift and drag coefficients in the components aligned with the hydrodynamic inflow, calculated from the OWT, and not with the nose-tail pitch line. However, the $\beta_{i}$-method still works under the assumption of the equivalent profile section. 


\subsubsection{Full-Scale CFD simulations}

In the marine industry, model testing has been the most precise alternative to know the propulsive requirement of a ship or the open water characteristics of a propeller. The continuous development of computational tools, such as CFD, are making a reality the possibility of simulate the OWT at full scale in a computer.

Direct full scale simulations are going to be a big step for the marine industry and will probably change completely the design process, when become the standard procedure. Some authors, such as Bulten and Stoltenkamp (2017), have already announced the promising future of full scale simulations. Today, it is still necessary to perform CFD simulations at model scale in order to validate the results obtained from CFD codes with experimental results, task that is not feasible when dealing with full scale simulations. Some examples of this kind of work are found in Ponkratov and Constanttinos (2015) and Vukcević et al. (2017).

\subsection{Numerical approaches to model laminar-turbulent transition}

Along this thesis the numerical approach used in all of the simulations is the Reynolds Average Navier Stokes (RANS) Equations. The RANS equations are discretized in space by the finite volume method using StarCCM+. StarCCM+ software has been used extensively in the hydrodynamic field and, particularly, when performing propellers computations (e.g. Bhattacharyya et al., 2015; Malan et al., 2009). A semi-implicit method, known as PIMPLE algorithm, is selected to handle the coupling of the Navier-Stokes equations and calculate the primitive variables, such as pressure and velocity at each time step. The temporal discretization solver used is a first order implicit scheme on the average fields.

The RANS equations for incompressible flow when considering a moving reference frame that rotates with constant angular velocity $\omega_{\mathbf{r}}$ can be written as:

$$
\begin{aligned}
\nabla \cdot \mathbf{v}_{\mathbf{r}} & =0 \\
\frac{\partial \mathbf{v}_{\mathbf{r}}}{\partial t}+\nabla\left(\mathbf{v}_{\mathbf{r}} \otimes \mathbf{v}_{\mathbf{r}}\right)+2 \omega_{\mathbf{r}} \times \mathbf{u}_{\mathbf{r}}+\omega_{\mathbf{r}} \times \omega_{\mathbf{r}} \times \mathbf{r} & =\frac{1}{\rho}\left(-\nabla p+\mu \nabla^{2} \mathbf{v}_{\mathbf{r}}\right)
\end{aligned}
$$

where $\mathbf{v}_{\mathbf{r}}$ is the relative velocity in the rotating reference frame, $p$ the fluid pressure, $\mathbf{r}$ is the radial 
vector from the rotation axis, $\rho$ and $\mu$ are the water density and dynamic viscosity, respectively.

Equations 2.1 and 2.2 have been complemented with the $k \omega-S S T$ turbulence model, which computes the turbulent kinetic energy $k$, the specific rate of dissipation $\omega$ and the eddy kinematic viscosity $\nu_{t}=k / \omega$ (see Wilcox, 2006).

In order to detect the different laminar and turbulent regions and be able to study the laminar to turbulent transition phenomenon, different approaches have been developed. The most efficient methods to model this phenomenon are:

- Stability theory approach.

- Low Reynolds number approach.

- The intermittency transport method with empirical correlation.

- The $\overrightarrow{v_{2}}-f$ model.

- The intermittency and vorticity Reynolds number approach, $\left(\gamma-R e_{\theta t}\right)$.

- The laminar kynetic energy approach, $\left(k-k_{L}-\omega\right)$.

- LES and DNS method.

A detailed explanation of each method can be found in Di Pasquale et al. (2009).

In this particular study, the $\gamma-\operatorname{Re} \theta$ transition model has been also added to the previous set of equations. Details of this transition model are presented in Section 2.2.1 and further information may be found in Menter et al. (2004, 2006), Langtry and Menter (2009) and Malan et al. (2009). The $\gamma-R e_{\theta t}$ correlation-based transition model is totally compatible with modern CFD codes based on unstructured meshes. Also, recently, this model has been extended in order to be able to capture stationary crossflow effects by Muller and Herbst (2014) and Langtry et al. (2015).

\subsubsection{Brief description of the $\gamma-R e_{\theta}$ transition model}

The $\gamma-R e_{\theta}$ transition model only computes local variables and gradients. This model solves two additional transport equations, one for the intermittency $\gamma$ and another for the transition onset momentumthickness Reynolds number $\overline{R e}_{\theta t}$.

The transport equation for the intermittency is coupled with the turbulence model, turning on the turbulent production or maintaining the laminar regime, depending on the intermittency value. The transport 
equation for $\overline{R e}_{\theta t}$ is an essential part of the model, being the interface between the experimental correlation and the intermittency equation. It is important to highlight that, in our case, the $k \omega-S S T$ was selected as the turbulence model, but, of course, any other alternative turbulence model could be also used.

The general equations for $\gamma-R e_{\theta}$ transition model are:

$$
\begin{aligned}
\frac{D \rho \gamma}{D t} & =P_{\gamma}-E_{\gamma}+\nabla \cdot\left[\left(\mu+\frac{\mu_{t}}{\sigma_{f}}\right) \nabla \gamma\right] \\
\frac{D \rho \overline{R e_{\theta t}}}{D t} & =P_{\theta} t+\nabla \cdot\left[\sigma_{\theta t}\left(\mu+\mu_{t}\right) \nabla \overline{R e_{\theta t}}\right]
\end{aligned}
$$

where $P_{\gamma}$ and $E_{\gamma}$ are the intermittency production and destruction terms, $\mu_{t}$ is the turbulent viscosity, $\sigma_{f}$ and $\sigma_{\theta t}$ are model constants and $P_{\theta} t$ is the $\overline{R e}_{\theta t}$ production term. A complete description of the model and its formulation, including a detailed explanation of the production and destruction terms and constants of the model, can be found in Malan et al. (2009).

An additional term has been included in a new empirical correlation in order to capture cross-flow effects (see Langtry et al., 2015). This term considers the stream-wise vorticity $\Omega_{S w}$ as an indicator of the local cross-flow strength $H_{C F}$ in the boundary layer.

$$
H_{C F}=\frac{y \Omega_{S w}}{U}
$$

Consequently, the new empirical correlation, including the cross-flow effects $R e_{\theta t S C F}$, is a function of the $H_{C F}$ and the surface roughness $h$. The full description of the new correlation, including production, destruction terms and constants can be found in Langtry et al. (2015).

\subsection{Artificial Neural Networks applied to the scaling problem on marine propellers}

An Artificial Neural Network (ANN) is a black box with a series of algorithms that tries to recognize underlying relationships in a dataset through a process that mimics the way the human brain operates. Artificial Neural Networks are a very interesting tool in a wide variety of fields, such as engineering, medicine, communication, etc. and has became recently a very popular tool, under the belief that it can be used everywhere. With respect to our specific area of study, transition and scaling problems on 
marine propellers, in the author's opinion Artificial Neural Networks have a potential application.

As already quoted, laminar to turbulent transition is a very complex phenomenon. We know that it occurs in a certain Reynolds number range, but we do not know the exact number because it depends on many parameters, e.g. the turbulent intensity in the flow, the roughness of the wall, the pressure gradient and others. According to this, the critical Reynolds number range varies depending on the application considered. However, the majority of current extrapolation procedures are based on the idea of scaling the friction forces between model and full scale Reynolds numbers, only considering the Re as the input parameter for friction lines extracted with empirical methods. Due to the complexity of the laminar-turbulent transition phenomenon an artificial neural network can be very useful to find the hidden influence of the geometry and the specific flow condition in an Open Water Test.

From the author's perspective, there are two possible applications of ANN in marine propeller extrapolation methods:

- Skin friction prediction, at model and full scale, based on Reynolds number and geometrical properties of the section to study, detailed in Section 2.3.1.

- Direct hydrodynamic coefficients extrapolation ratio prediction, between model and full scale $C F D$ simulations, based on geometrical characteristics of the propeller and flow condition of the model tests. Detailed at Section 2.3.2

For the purpose of this thesis, the particular type of neural network called Multilayer Perceptron (MLP) has been used. The MLP is a mathematical function that maps an inputs set to certain output values by composing many simple functions. A more detailed explanation of the MLP and the basic concept of the ANN may be found in Goodfellow et al. (2016). The MLP basic scheme for two hidden layer (HL) of neurons is presented in Figure 2.1.

The detailed generation of the specific MLP for the skin friction prediction based on ANN is presented in Appendix D.2.

\subsubsection{Friction coefficient prediction}

In the most common extrapolation methods, in order to apply corrections to the OWT results, the difference between the flat plate friction coefficient for model and full scale local Reynolds number on the propeller is used. The main question that still remains unsolved, due to the complexity of the physics involved, is the laminar-turbulent transition onset identification, which is a crucial factor to calculate the 


\section{Input Layer Hidden Layers $\quad$ Output Layer}

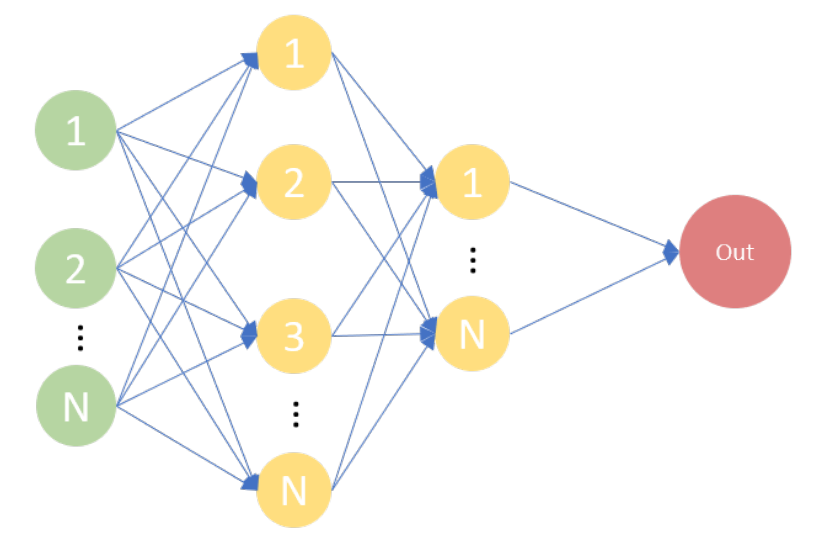

HL $1 \quad H L 2$

Figure 2.1: $M L P$ basic scheme.

flat plate friction coefficient. With the aim to improve the laminar-turbulent transition onset identification, it is possible to apply the MLP based on CFD simulations, extracting the radial data and integrating the tangential component of the wall shear stress to obtain the skin friction over the sections of the propeller. This results can be used to train a neural network for local friction coefficient prediction.

A conceptual scheme of the basic actual scaling flow chart for marine propellers is presented in Figure 2.2 where the input to estimate the friction forces of the equivalent flat plate, only depends on the Reynolds number of a particular section of the propeller. The specific formulas, represented by arrows in the figure, depend on the extrapolation method used.

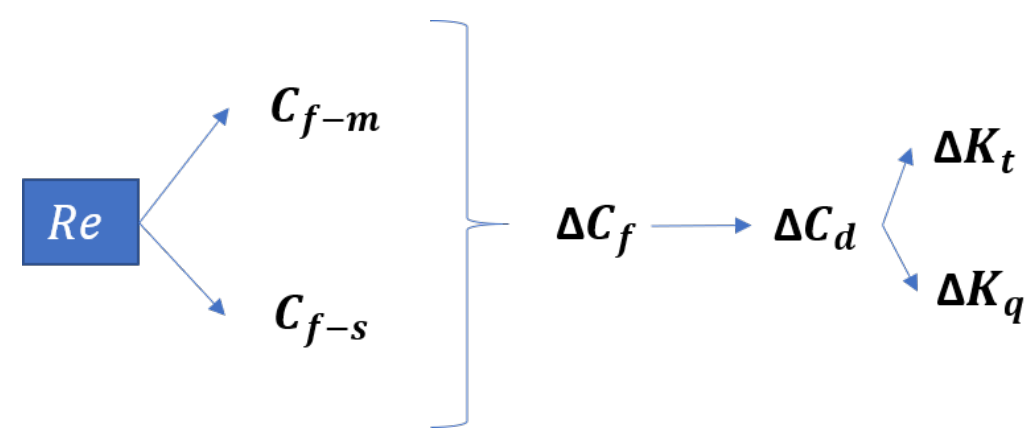

Figure 2.2: Actual basic scaling scheme for the skin friction prediction.

The proposed new scaling flow chart with the usage of the MLP for the estimation of the $C_{f}$ is presented in Figure 2.3. In this case, as example, the inputs of the MLP are the Reynolds number, the thickness-chord ratio $T H C R$, the camber-chord ratio $F C R$ and the profile family of the propeller sec- 
tions.

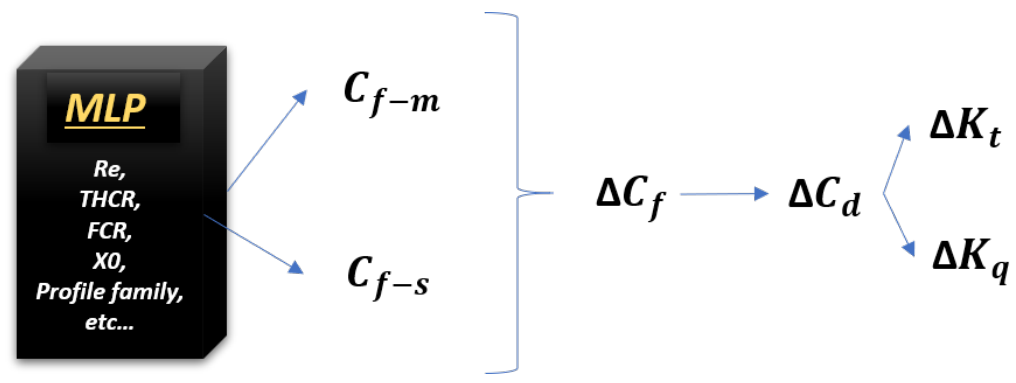

Figure 2.3: Proposed scaling scheme for the skin friction prediction with the usage of $M L P$.

Three things are necessary in the training process of a MLP capable to give accurate results and generalize the problem to any kind of propeller: accurate CFD results, quantity and variety of the propellers dataset. An MLP trained with CFD data can be as good, representing the reality, as the CFD data used to train the network. All the assumptions will be indirectly included in the results extracted from the trained network.

\subsubsection{Direct $K_{t}$ and $K_{q}$ extrapolation ratio}

The second utility of Artificial Neural Networks in the marine propeller extrapolation problem is to create an MLP that takes as entries the parameters of each section $X i$, to directly generate the scaling factors $R_{K t}$ and $R_{K q}$ defined by Equation 2.6. This parameters are divided into the ones that completely describe the geometry of the propeller, such as the thickness-chord ratio, the camber-chord ratio, etc.., and those that take into account the flow conditions of the OWT,

$$
R_{K t}=\frac{K_{t_{f}}}{K_{t_{m}}} \quad R_{K q}=\frac{K_{q_{f}}}{K_{t_{m}}}
$$

The interesting part of obtaining directly the $K_{t}$ and $K_{q}$ extrapolation ratios is that no assumptions are included, and the scaling effects in lift and drag forces remains implicitly in the scaling factors. For this purpose, it is expected a large amount of propeller dataset to get an optimum artificial neural network capable to generalize the problem and give accurate results. The amount of data for the input is expected to be larger than in the previous alternative, mainly because a general description of the propeller is needed and because higher non-linearities in the scaling coefficients are expected due to 
the inclusion of the drag and lift scale effects.

The scaling flow chart is represented in Figure 2.4 and an example of a possible scheme of the MLP is shown in Figure 2.5.

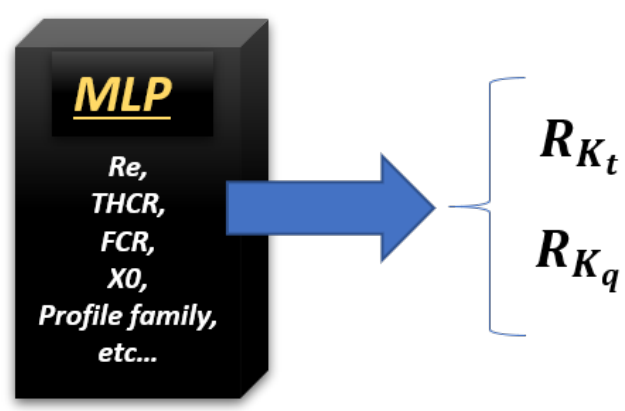

Figure 2.4: Actual basic scaling scheme for the direct scaling of $K_{t}$ and $K_{q}$.

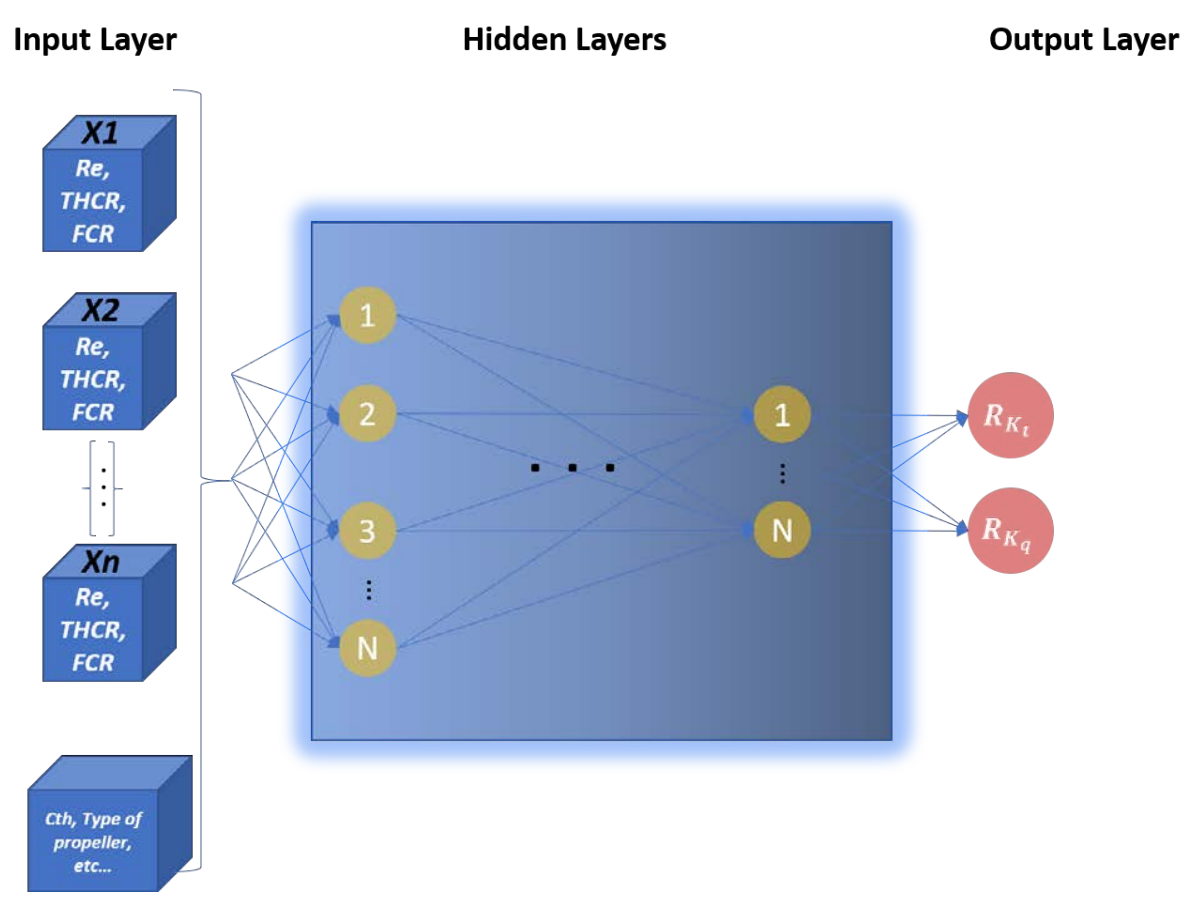

Figure 2.5: Proposed scheme of the $M L P$ for the direct estimation of the scaling factors $R_{K t}$ and $R_{K q}$. 
CHAPTER 2. GENERAL CONSIDERATIONS 


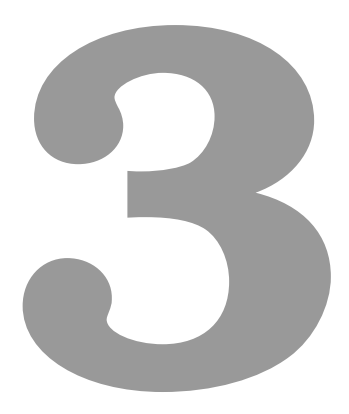

Results

\section{Contents}

3.1 Cross-flow specific study: M2355 profile . . . . . . . . . . . . . . . . . 23

3.2 Different turbulent approaches comparison at model scale propellers simulations . 28

3.3 New friction line from CFD results . . . . . . . . . . . . . . . . . . 40

3.4 New Form factor and lift scale effects . . . . . . . . . . . . . . . . . . 47

3.5 Analysis of different extrapolation methods. . . . . . . . . . . . . . . . . . . 54 
CHAPTER 3. RESULTS 


\subsection{Cross-flow specific study: M2355 profile}

Transition from laminar to turbulent due to cross-flow instabilities are the most important transition mechanism at low angles of attack when the flow is not parallel to the chord line of the profile, see Saric et al. (2003).

In this section, a comparison between the original $\gamma-R e_{\theta}$ model and the new version that includes transition prediction due to cross-flow effects is going to be presented in order to observe the differences between these models. As the original $\gamma R e_{\theta}$ transition model was designed for by-pass and natural (TS) transition, we expect a certain improvement in transition onset prediction when the cross-flow term is included in the model.

For this particular study, the geometry chosen is the M2355 profile, due to the availability of experimental results. It is not the typical profile to generate the blade of a marine propeller, but it is still valid to obtain some conclusions to the importance of the cross-flow modelling.

\subsubsection{Set-up}

The M2355 is a deeply studied profile used as a test case by the aircraft company Airbus. This particular profile is going to be studied for a constant sweep angle and different Angle of Attack (AoA). In Table 3.1 the main parameters of the simulation are exposed.

The improvements when the new correlation to capture cross-flow is applied were compared to the conventional $\gamma R e_{\theta}$ model for different angles of attack. The level of ambient turbulent intensity has been varied to test the influence of this parameter on the transition onset.

Firstly, a mesh convergence process has been carried out using the transition onset point as an indicator of the mesh convergence. The mesh convergence has been carried out at $A \circ A=0^{\circ}$ and zero swept angle. The mesh convergence test is performed for five different meshes, see Figure 3.1. We observed that when the mesh refinement is increased, the transition point moves toward the trailing edge in both suction and pressure sides $\left(S_{s}\right.$ and $\left.P_{s}\right)$. The differences in the transition location was less than $1 \%$ when the two most refined meshes are compared. 


\begin{tabular}{|l|c|}
\hline \multicolumn{2}{|c|}{ M2355 Profile } \\
\hline Profile & 3D \\
\hline Dimensions & Unsteady \\
\hline Time assumption & Compressible \\
\hline Compressibility assumption & Yes \\
\hline Periodicity assumption & 0.435 \\
\hline Chord [m] & $5.0 \cdot 10^{6}$ \\
\hline Reynolds & $0^{\circ}-3^{\circ}$ each $0.5^{\circ}$ \\
\hline Angle of attack & $25^{\circ}$ \\
\hline Sweep angle & $\gamma R e_{\theta}$ and $\gamma R e_{\theta}+C_{r o s s}-f l o w$ \\
\hline Turbulence Models & $0.07 \%-0.5 \%$ at leading edge \\
\hline Turbulent intensity & minimum of the Skin Friction curve \\
\hline Transition Onset prediction criterium & \\
\hline
\end{tabular}

Table 3.1: Main parameters of the M2355 profile simulation.

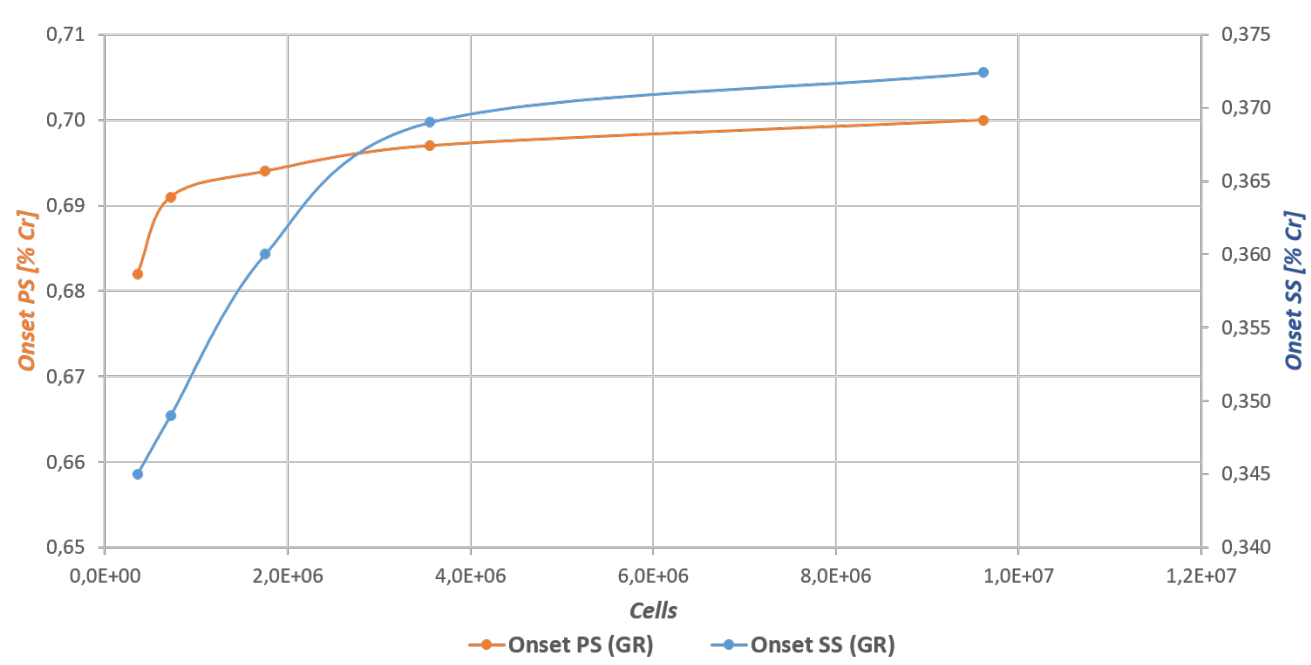

Figure 3.1: Mesh convergence test where the transition onset criteria is monitored on suction and pressure sides. 


\subsubsection{Results}

In this subsection, the laminar-turbulent transition predictions are presented for the M2355 profile. Although not many experimental results in the presence of crossflow and different angles of attack are found in the literature, results from the Airbus Group Innovations experimental database are used for comparison. The experimental transition locations were based on two different experimental techniques, Temperature Sensitive Painting (TSP) and Hotfilm probes. The 3D computational geometry was built by the extrusion of a 2D profile using periodic boundary conditions for the lateral planes.

The main objective of this study is to evaluate the influence of the new additional cross-flow correlation $\left(R e_{\theta S C F}\right)$ in the original $\gamma R e_{\theta}$ transition model, which takes into account cross-flow effects. Details about the model can be found in Section 2.2.1 and the conditions for this simulation can be found in Section 3.1.1. The transition location criterion used to locate the transition onset is identified with the minimum of the friction coefficient curve. This criterion is applied independently on both the suction and pressure sides at the middle cross section.

In Figures 3.2 and 3.3, the transition onset location is represented against the angle of attack, with and without the cross-flow correlation, when the turbulence intensity parameter is $T u=0.07 \%$ at the leading edge.

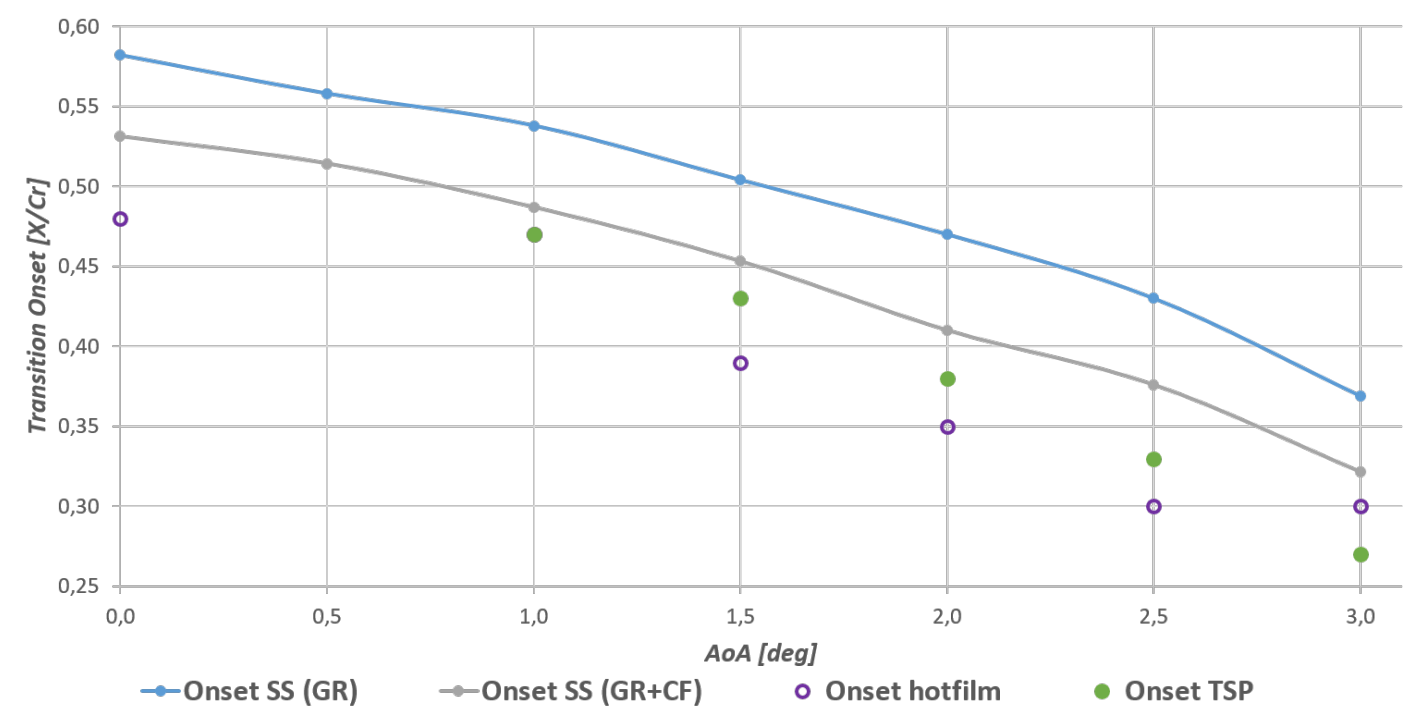

Figure 3.2: Transition onset on suction side for different $A o A$.

It can be observed in Figure 3.2 that, on the suction side, the inclusion of the cross-flow term improves the transition location prediction and moves the results closer to the experimental values. In the 


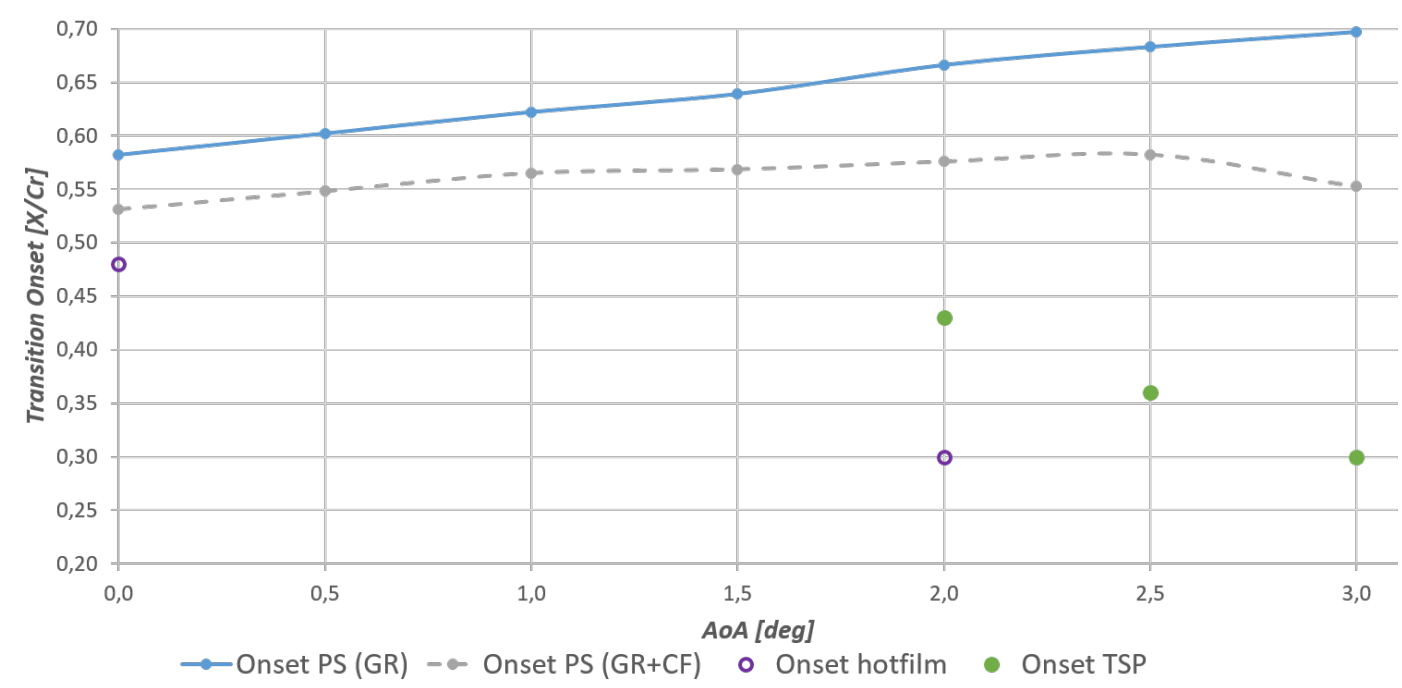

Figure 3.3: Transition onset on pressure side for different $A o A$.

pressure side, the experimental evidence in the absence of cross-flow is that the transition onset moves toward the trailing edge when the angle of attack is increased, see Menter et al. (2006). This tendency changes completely when experiments are performed in the presence of cross-flow, where the onset position moves toward the leading edge as the angle of attack is increased. In Figure 3.3, it is possible to appreciate both tendencies from CFD results. This behavior could be explained by the fact that when the angle of attack grows, cross-flow effects on the pressure side are increased, see Dagenhart (1992) and Saric et al. (2003). This important change on the pressure side is not captured at all when the model does not include the cross-flow modelling. Although the computational results on the pressure side are not close enough to the experimental values, they improve when the cross-flow term is added.

As the turbulence intensity value measured in the wind tunnel was not confirmed, Figure 3.4 presents a study where typical values of turbulent intensity in wind tunnels have been used in order to be compared to the experimental values. For all the turbulent intensities values in Figure 3.4, the cross-flow correlation was used. Two different methods to control the turbulence intensity were used. In the first one, the turbulence intensity value decays from $T u=1 \%$ at the inflow to $T u=0.07 \%$ at the leading edge of the profile. In the other case, a source of turbulent intensity all over the domain in the range $T u=0.1 \%-0.5 \%$ was used for all range of angles of attack. On the suction side, results indicate that larger turbulence intensity values move the transition onset towards the leading edge. Differences between experimental and computational values are reasonably small for the case when turbulence intensity is around $T u=0.1 \%$, which is a typical value in wind tunnels. For the pressure side, similar changes in the turbulent intensity did not improve the overall results. The slopes of the curves do not 


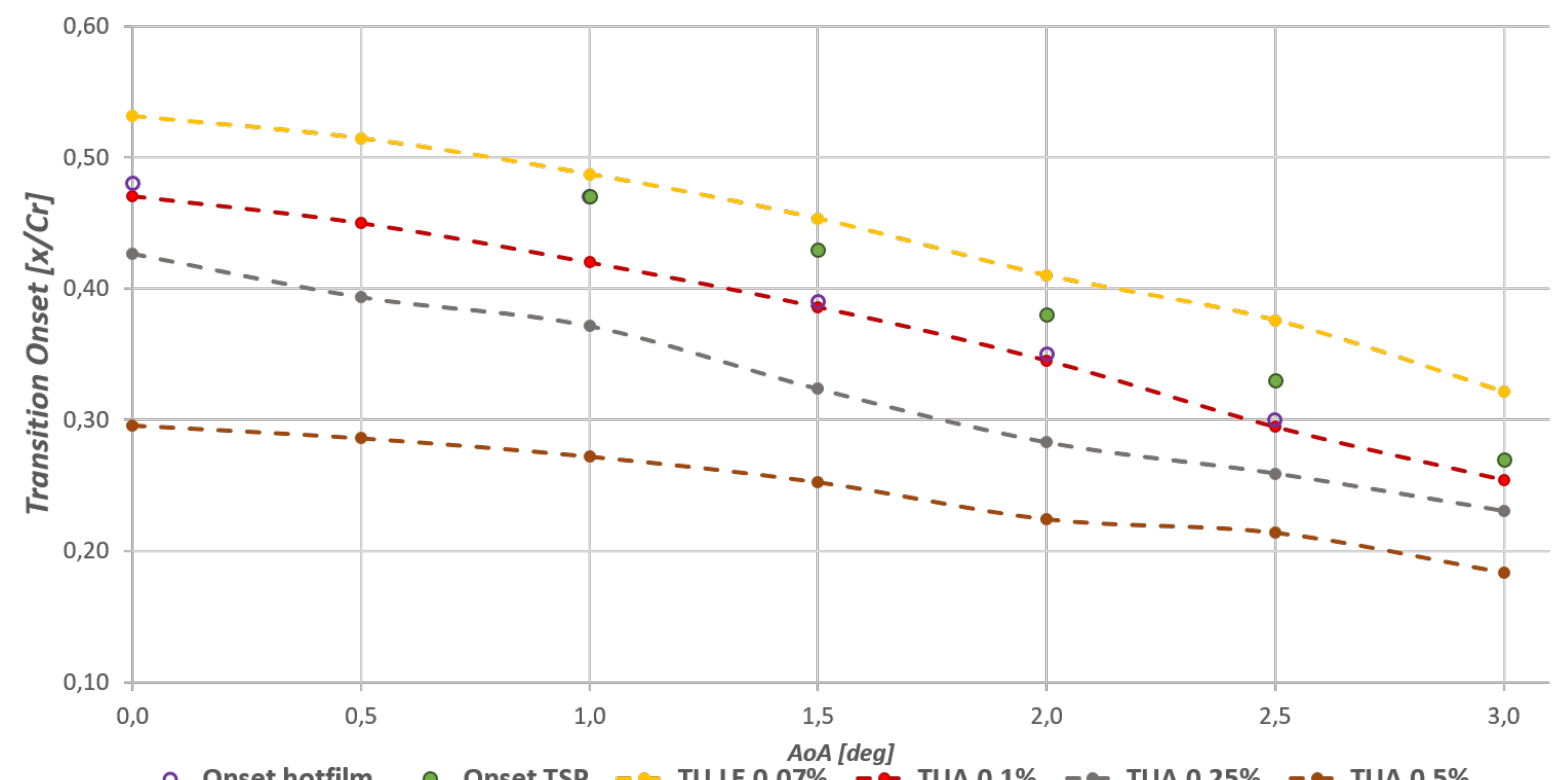

$\circ$ Onset hotfilm $\circ$ Onset TSP $=\bullet$ TU LE $0.07 \%$ - - TUA $0.1 \% \quad-\circ$ TUA $0.25 \%$ - - TUA $0.5 \%$

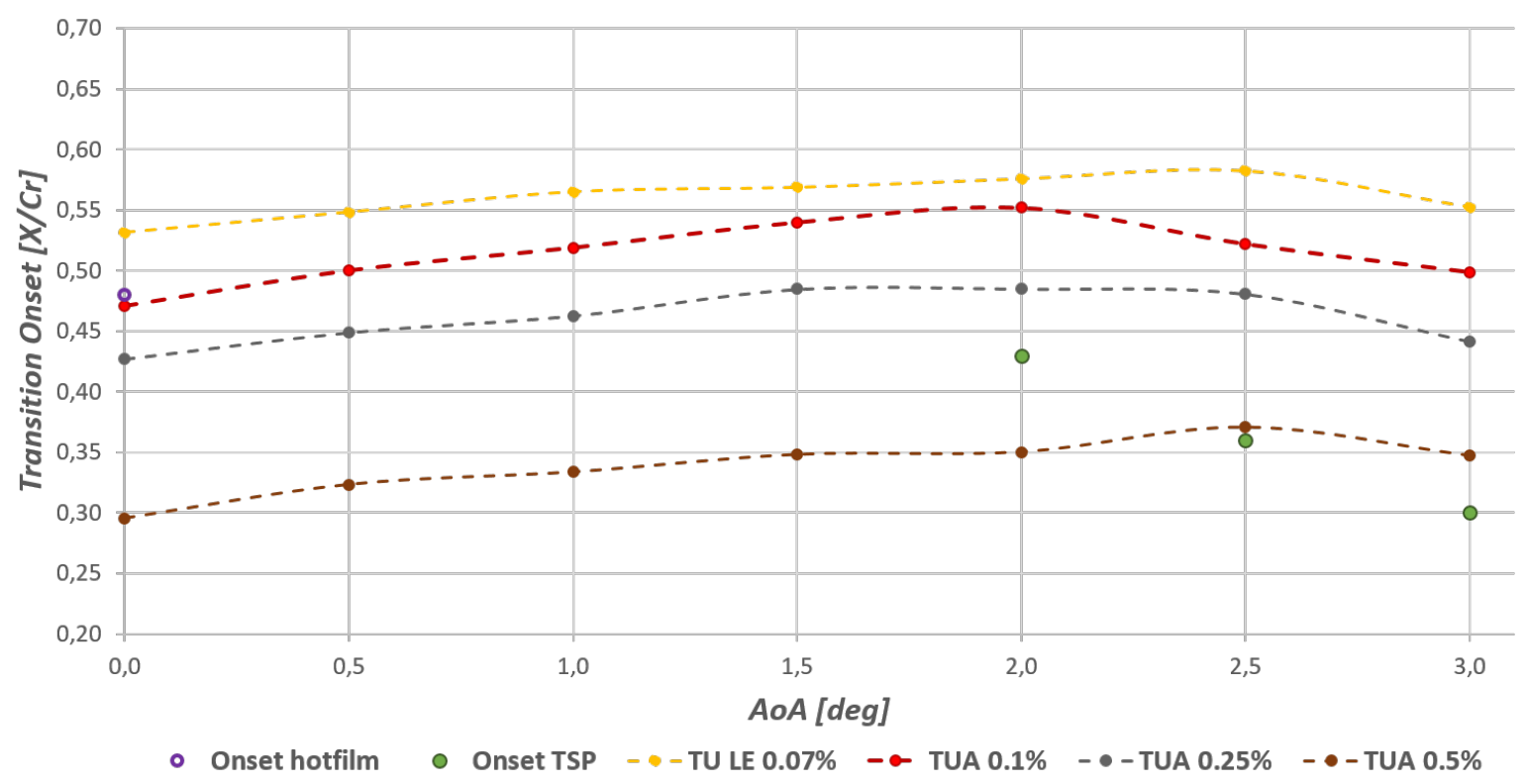

Figure 3.4: Transition onset in suction side (top) and pressure side (bottom) for different $A o A$ and different values of the turbulence intensity.

behave correctly even if the value of turbulent intensity is varied. Consequently, the cross-flow modelling should be improved on the pressure side in order to become closer to the experimental values. 


\subsection{Different turbulent approaches comparison at model scale pro- pellers simulations}

In this subsection, a comparison of different turbulence models for the OWT simulation in marine propellers is going to be presented. The turbulence models used in this section are the well-known $K \omega-S S T$ (Fully turbulent), the $\gamma R e_{\theta}$ (Laminar-Turbulent) and the $\gamma R e_{\theta}+$ Cross - Flow (LaminarTurbulent). For the pourpose of comparing the effects of each model, three ship propeller geometries are considered: the two International Towing Tank Conference (ITTC) benchmark propellers (VP1304 conventional and P1727 non-conventional geometry) and the new generation of (Contracted and Loaded Tip) CLT®propeller (Gonzalez-Adalid et al., 2016; Gaggero et al., 2016). Although two of them are variable pitch propellers, this study has been carried out at fixed pitch angle. All of the propellers have been experimentally tested at model scale, for different advanced ratios $(\mathrm{J})$, and the numerical results obtained will be compared.

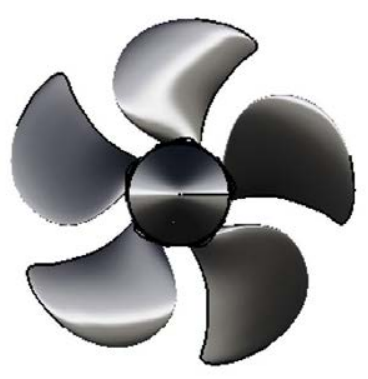

a

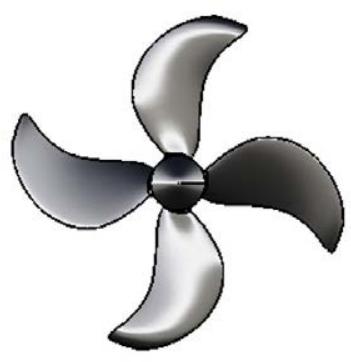

b

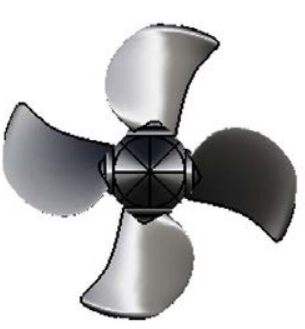

C

Figure 3.5: Geometries of the three propellers studied: VP1304 (a), P1727 (b) and the new generation of CLT propeller (c).

The VP1304 propeller is a five blade conventional propeller while the P1727 and CLT propellers have only four blades and are a Tip-Raked and a CLT propeller, respectively, see Figure 3.5. The main geometric characteristics of the propellers are in Table 3.2.

In all simulations, an axisymmetric boundary condition has been used, simulating only one propeller blade. To avoid mesh rotation or re-meshing at each time step, the rotational term is added to the NavierStokes equations being the propeller fixed to the computational domain, transforming the equations to consider a Moving Reference Frame. The complete process of the mesh convergence followed to obtain an accurate and consistent mesh is presented in Appendix C 


\begin{tabular}{|l|l|c|c|c|}
\hline \multicolumn{2}{|l|}{} & VP1304 & P1727 & CLT \\
\hline Diameter [mm] & $D$ & 250.0000 & 238.6407 & 208.0000 \\
\hline Pitch at 0.75R [mm] & $P_{0.75}$ & 407.3804 & 192.0150 & 242.5280 \\
\hline Chord at 0.75R [mm] & $C r_{0.75}$ & 106.3476 & 55.6172 & 71.1420 \\
\hline Thickness at 0.75R [mm] & $T h_{0.75}$ & 3.7916 & 2.9329 & 2.6321 \\
\hline Area Ratio & $A e / A o$ & 0.779 & 0.4438 & 0.565 \\
\hline Blades & $z$ & 5 & \multicolumn{2}{|c|}{} \\
\hline
\end{tabular}

Table 3.2: Main geometric characteristics of the ship propellers studied.

The range of the advance coefficients $J$ studied depends on the design point of each propeller and the range studied at each experimental campaign. Consequently, different ranges are used for each propeller. The conditions studied are summarized in Table 3.3.

\begin{tabular}{|l|c|c|c|}
\hline & VP1304 & P1727 & CLT \\
\hline Time asumption & \multicolumn{3}{|c|}{ Steady } \\
\hline Rotation asumption & \multicolumn{3}{|c|}{ MRF asumption } \\
\hline Axisymmetric asumption & \multicolumn{3}{|c|}{ Yes } \\
\hline Diameter $D$ [m] & 0.2500 & 0.2387 & 0.2080 \\
\hline Advance Ratio $J$ & {$[0.6,0.8,1.0,1.2,1.4]$} & {$[0.1,0.3,0.5,0.7,0.9]$} & {$[0.6,0.7,0.8,0.9,0.95]$} \\
\hline Revolutions $n$ [rps] & 15.0 & 18.0 & 15.0 \\
\hline Reynolds at $0.75 \mathrm{R} R e_{0.75}$ & $9.009 \cdot 10^{5}$ & $5.0789 \cdot 10^{5}$ & $4.8747 \cdot 10^{5}$ \\
\hline
\end{tabular}

Table 3.3: General parameters for the different propellers. Reynolds are calculated at $J=1.0$ for the VP1304, $J=0.5$ for the P1727 and $J=0.8$ for the CLT.

\subsubsection{P1727 Tip-Rake Propeller}

In the following, the non-conventional P1727 Tip-Rake propeller results are presented. The P1727 Tip-Rake propeller was selected by the ITTC as a benchmark case for the scaling study of non-conventional propellers in 2017. The experimental measurements performed in the SVA Towing Tank are available for comparison in SVA. The range of advance coefficients studied is from $J=0.1$ to $J=0.9$. For this 
case, the mesh has 8193266 cells and 20 prism layers in the boundary layer. Three different turbulence models were used, a fully turbulent $K \omega-S S T$ model, a $\gamma R e_{\theta}$ transition model that controls the regions where the $K \omega-S S T$ model is used and, lastly, a third variant where the transition model includes the modelization of the cross-flow transition mechanism.

In Figure 3.6 we can observe the efficiency values $\eta_{0}$ in the open water curve given by Equation 3.1.

$$
\eta_{0}=\frac{K_{t}}{2 K_{q} \pi} J
$$

Although for $J<0.5$ the presence of transition models is not relevant, important differences can be observed for $J \geq 0.5$ where the $\gamma R e_{\theta}$ with and without transition model approximate the experimental values quite accurately. The only exception is $J=0.9$ which is out of the propeller's operative range. In Table 3.4, the difference between each method and the experimental values $\left(\Delta \eta_{0}\right)$ is presented. We can observe that when transition models are used, the differences with the experimental values are always below $2 \%$ for the whole range of advance coefficients. The highest difference is found for the highest advance coefficient $J=0.9$, where the propeller efficiency curve decreases drastically. The $J=0.9$ value corresponds to a working point far from the design point, which implies larger amounts of detached flow around the profiles and, consequently, a more complex hydrodynamic scenario to simulate.

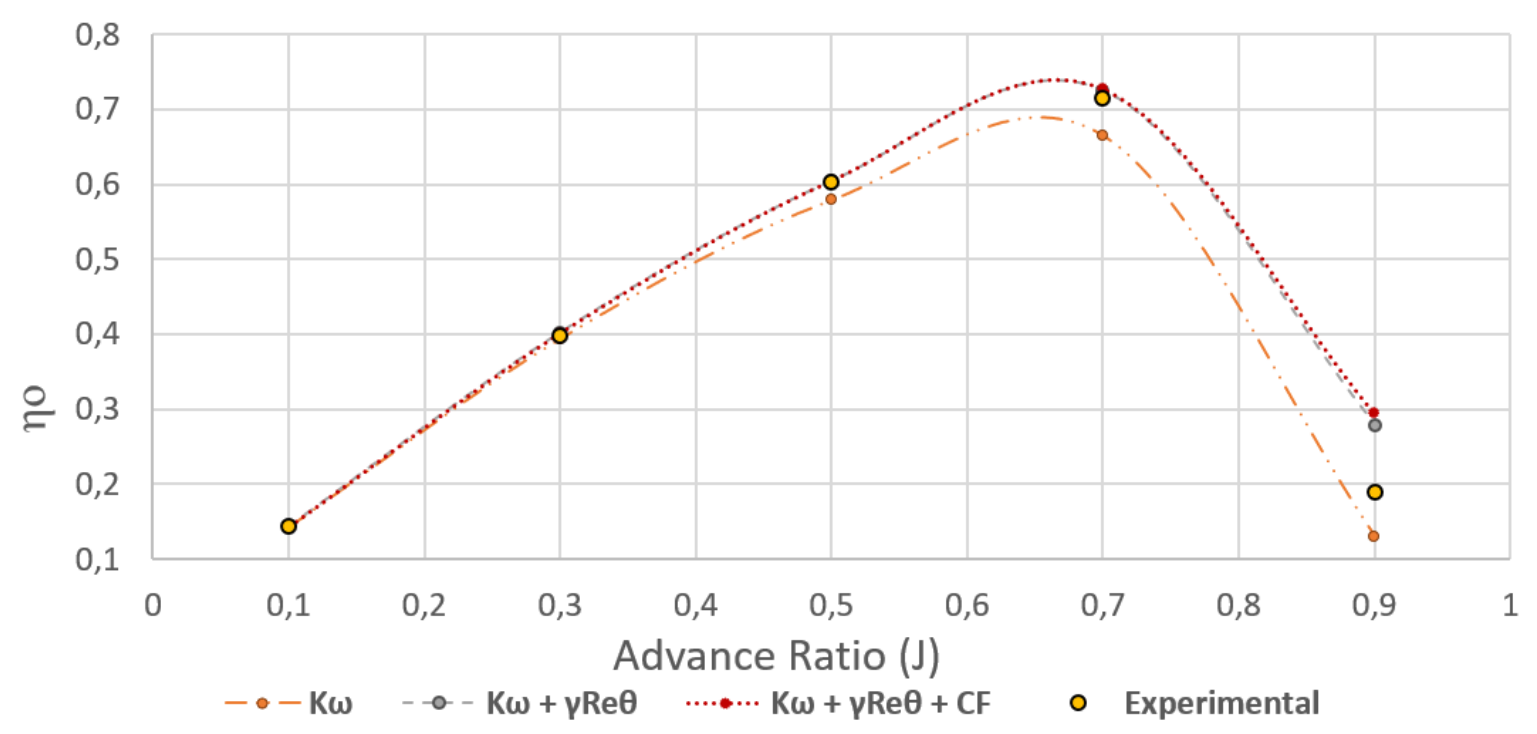

Figure 3.6: Differences on the efficiency values with respect to the experimental values for the P1727 propeller.

Figure 3.7 presents the differences in $K_{t}$ and $K_{q}$ values with respect to the experimental results for a 


\begin{tabular}{|c|c|c|c|}
\hline $\mathrm{J}$ & $K \omega-S S T \Delta_{\eta_{0}}[\%]$ & $\gamma \operatorname{Re}_{\theta} \Delta_{\eta_{0}}[\%]$ & $\gamma$ Re $e_{\theta}+$ Cross - Flow $\Delta_{\eta_{0}}[\%]$ \\
\hline 0.1 & -1.43 & -0.38 & -0.58 \\
\hline 0.3 & -1.25 & +0.96 & +0.70 \\
\hline 0.5 & -3.89 & +0.37 & +0.36 \\
\hline 0.7 & -6.92 & +1.50 & +1.71 \\
\hline 0.9 & -31.12 & +47.92 & +55.53 \\
\hline
\end{tabular}

Table 3.4: Differences on the efficiency values with respect to the experimental values for the P1727 propeller.

range of advance coefficients close to the design point of the propeller. It can be observed that transition models improve the results in terms of thrust and torque, and not only in terms of the global efficiency of the propeller.
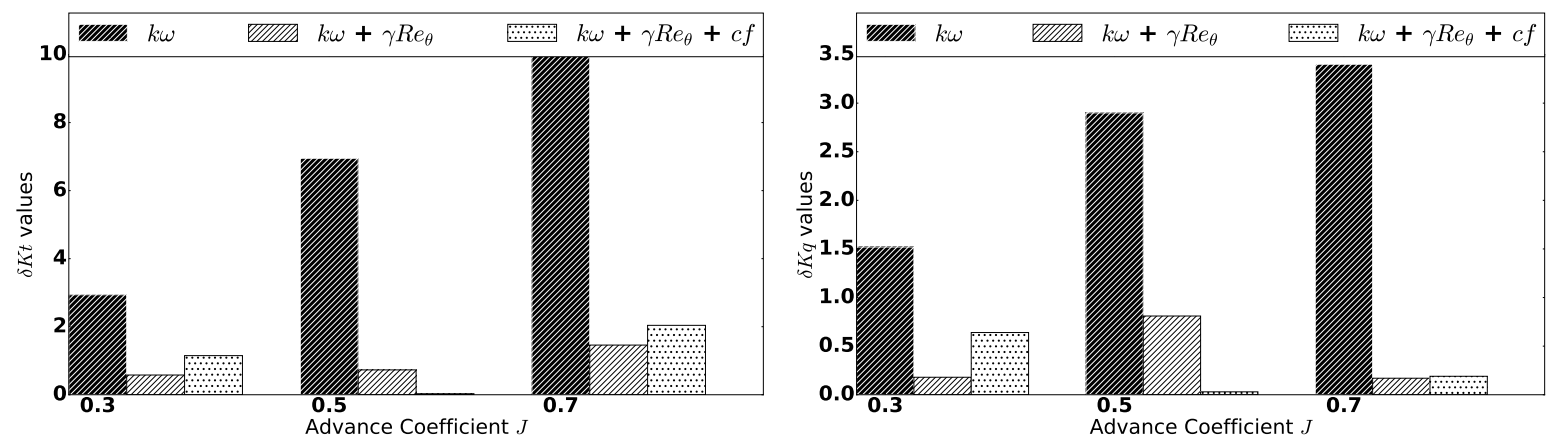

Figure 3.7: Differences in dimensionless thrust and torque coefficients with respect to the experimental values for the P1727 propeller.

In order to perform a local study of the transition phenomena, the propeller blade was divided into seven sections according to the ITTC prescription, see Figure 3.8. On each section, the skin friction coefficient $C_{f}$ has been averaged and represented along the non-dimensional radius $r / R$, as exemplified in Figure 3.9. Results show a clear improvement when the transition models are added to the simulation decreasing the averaged skin friction coefficients for all sections. Negligible differences are seen in this case when the cross-flow transition model is included.

When the intermittency plots with and without the cross-flow term are compared at $J=0.5$, close to the design advance coefficient $J=0.56$, small differences are observed if the cross-flow is modelled, as seen in Figure 3.10. 


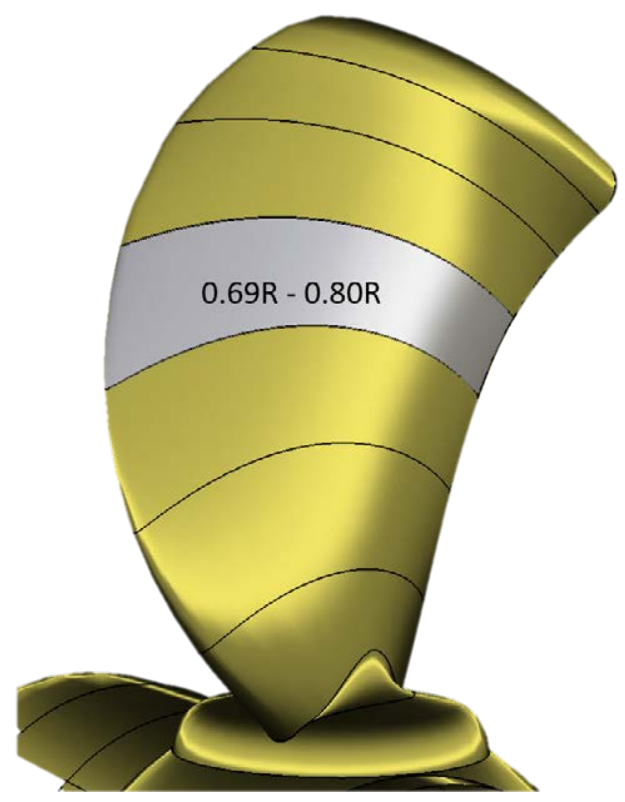

Figure 3.8: Scheme of the different sections used for the local transition study.

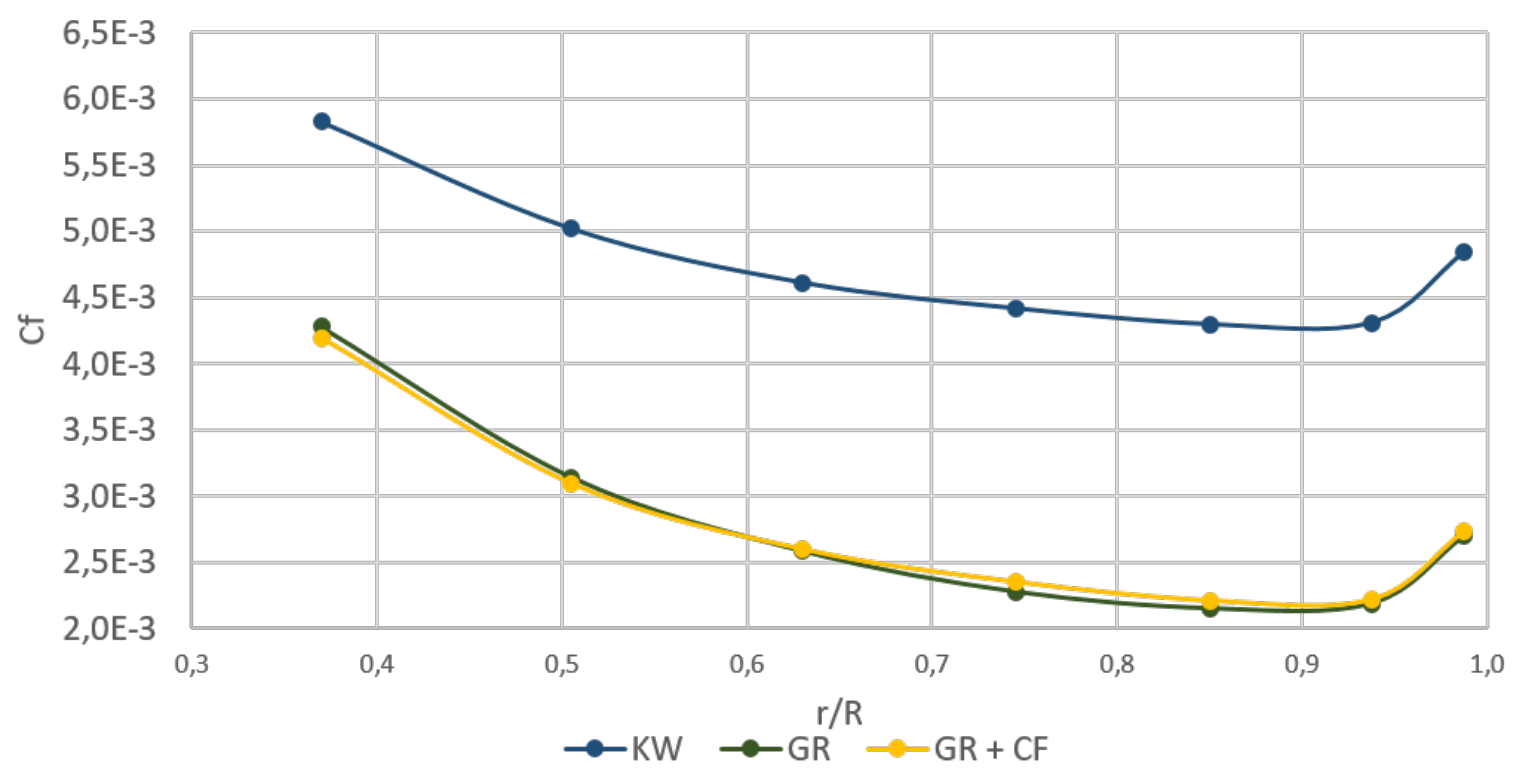

Figure 3.9: Skin friction comparison for different dimensionless radius for P1727 ITTC benchmark propeller.

Figure 3.11 shows the streamlines and the skin friction coefficients over suction and pressure sides for the three methods studied. As before, when fully turbulent flow is assumed, the centrifugal component of the streamlines is not significantly intense and the streamlines follow a radial direction. When the 


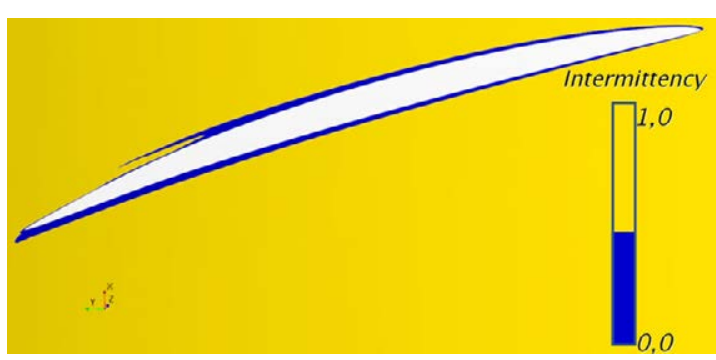

(a) $\gamma-R e_{\Theta}$

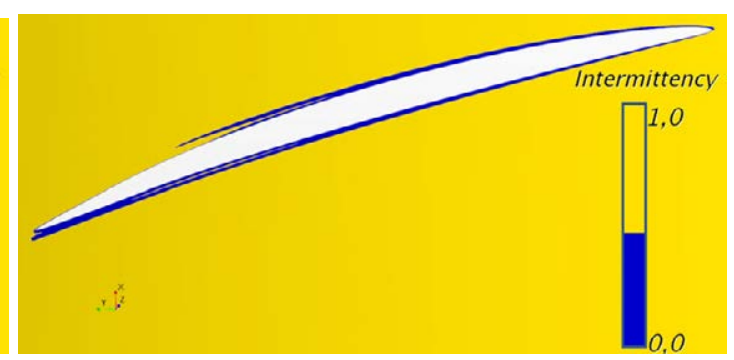

(b) $\gamma-\operatorname{Re}_{\Theta}+$ CrossFlow

Figure 3.10: Intermittency contour plot at $r / R=0.9375$ of the non-conventional P1727 Tip-Rake Propeller for $J=0.5$.

transition models are included, the slope of the streamlines changes noticeably due to the centrifugal component. This could be explained in terms of the tendency to rotate that the streamlines have in the laminar region where the skin friction coefficients are lower, comparing to the turbulent areas with higher friction. Near the trailing edge, an accumulation of streamlines following the radial direction is appreciated in both transition models. When the cross-flow term is added to the simulation, higher skin friction coefficients are observed close to the trailing edge.

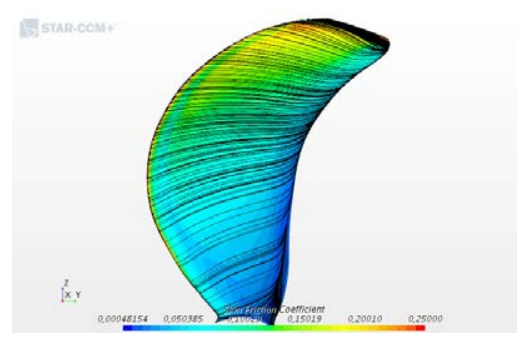

(a) $K \omega$

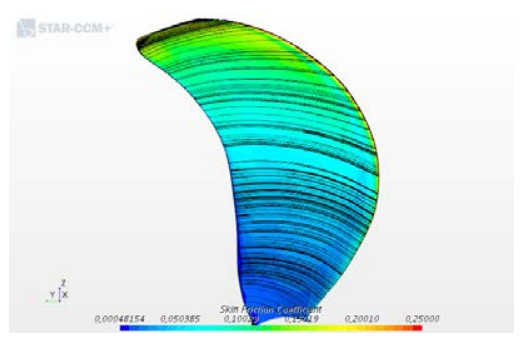

(d) $K \omega$

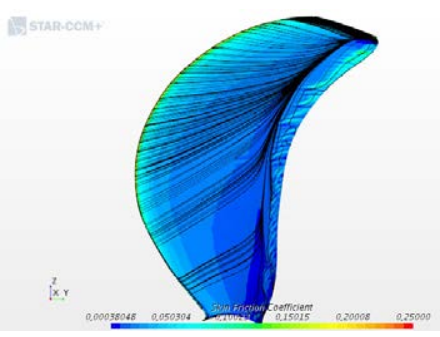

(b) $\omega+\gamma R e_{\theta}$

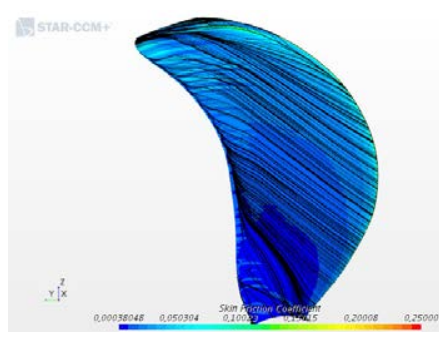

(e) $\omega+\gamma R e_{\theta}$

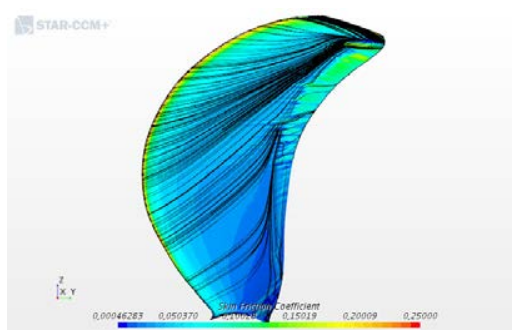

(c) $K \omega+\gamma R e_{\theta}+C F$

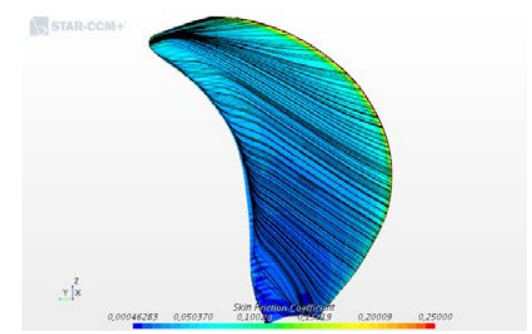

(f) $K \omega+\gamma R e_{\theta}+C F$

Figure 3.11: Skin friction contour plot and streamlines representation over the suction (top) and pressure (bottom) sides for the P1727 ITTC Benchmark propeller at $J=0.5$.

As can be seen, local plots, such as Figure 3.11, show complementary information compared to 
sectional plots recommended by the ITTC, such as Figure 3.9, where the average process hides local information.

\subsubsection{New generation of CLTßPropeller}

In the following, results of the new generation of $\mathrm{CLT} \AA$ propeller are presented. This propeller was experimentally tested in 2016 in the CEHIPAR towing tank facilities. The advance coefficients studied are from $-25 \%$ to $+15 \%$ of the design point $J \approx 0.8$. For this case, the mesh has 6920765 cells and 20 prism layers in the boundary layer. Three different turbulence models were used, a fully turbulent $K \omega-S S T$ model, a $\gamma R e_{\theta}$ transition model that controls the regions where the $K \omega-S S T$ model is used and, lastly, a third variant where the transition model includes the modelization of the cross-flow transition mechanism.

In Figure 3.12, we can see the open water curve for different transition scenarios. The transition models clearly improve the agreement with the experimental values compared to the fully turbulent case. As in the previous propeller, the largest differences are found again for the highest advance coefficients. In order to quantify these differences, Table 3.5 shows a relative comparison between each method and the experimental results obtained from the CEHIPAR model basin. According to Table 3.5, the transition models clearly improve the result in terms of the efficiency of the propeller, being almost below two percent.

\begin{tabular}{|c|c|c|c|}
\hline $\mathrm{J}$ & $K \omega-S S T \Delta_{\eta_{0}}[\%]$ & $\gamma R e_{\theta} \Delta_{\eta_{0}}[\%]$ & $\gamma$ Re $e_{\theta}+$ Cross - Flow $\Delta_{\eta_{0}}[\%]$ \\
\hline 0.60 & -3.0 & 0.0 & 0.0 \\
\hline 0.70 & -4.2 & -0.4 & -0.1 \\
\hline 0.80 & -6.1 & 0.4 & 0.2 \\
\hline 0.85 & -7.5 & 1.8 & 2.0 \\
\hline 0.90 & -10.0 & 1.9 & 2.2 \\
\hline
\end{tabular}

Table 3.5: Open Water results for new generation of CLT propeller with respect to the experimental values.

Figure 3.13 presents the differences in $K_{t}$ and $K_{q}$ values with respect to the experimental results for different advance coefficients. In this case, all models under-predict overall results for thrust. Although the fully turbulent model performs better than the transition models for the torque coefficient, these differ- 


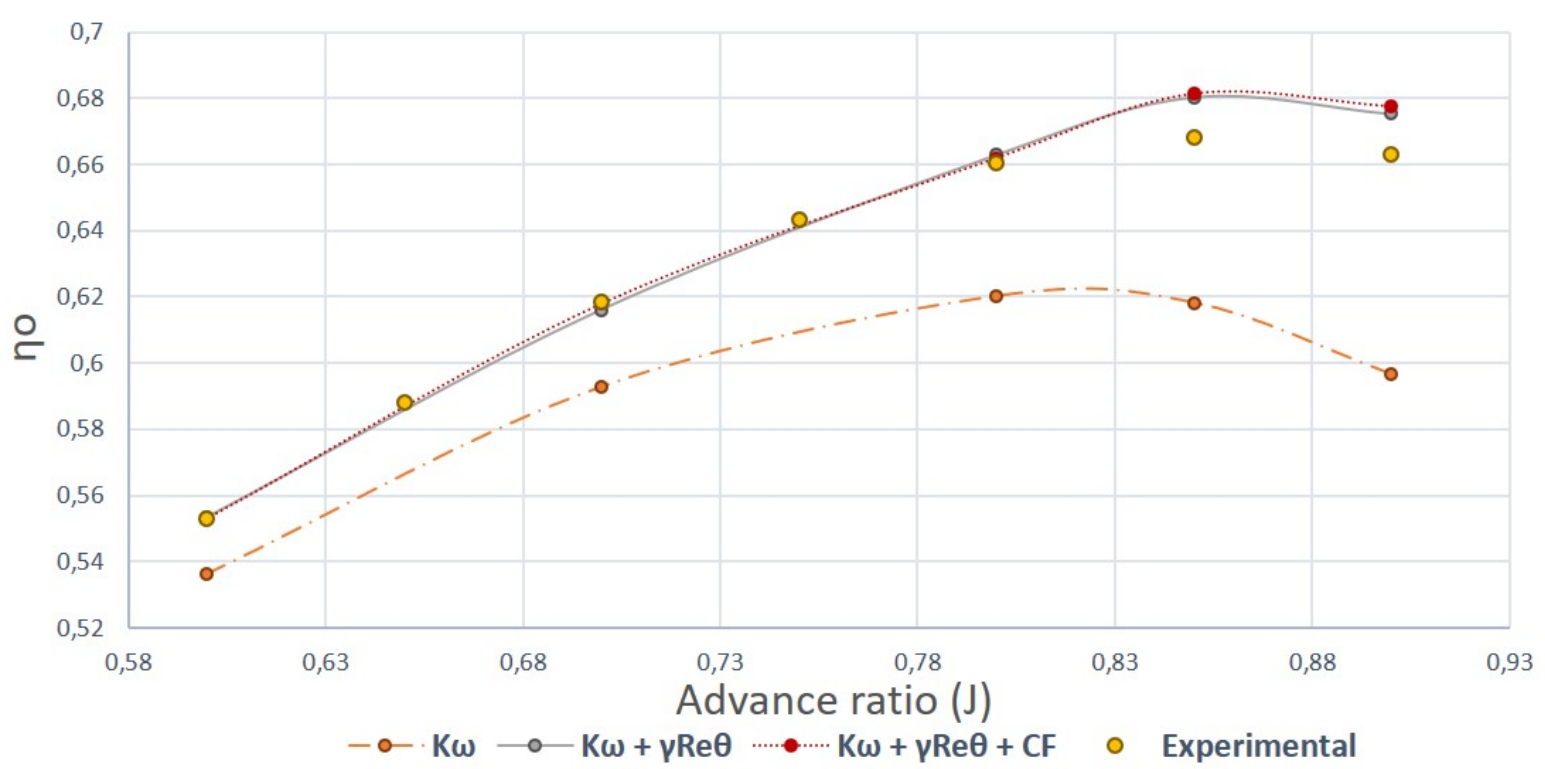

Figure 3.12: Efficiency values of the open water test for the CLT propeller.
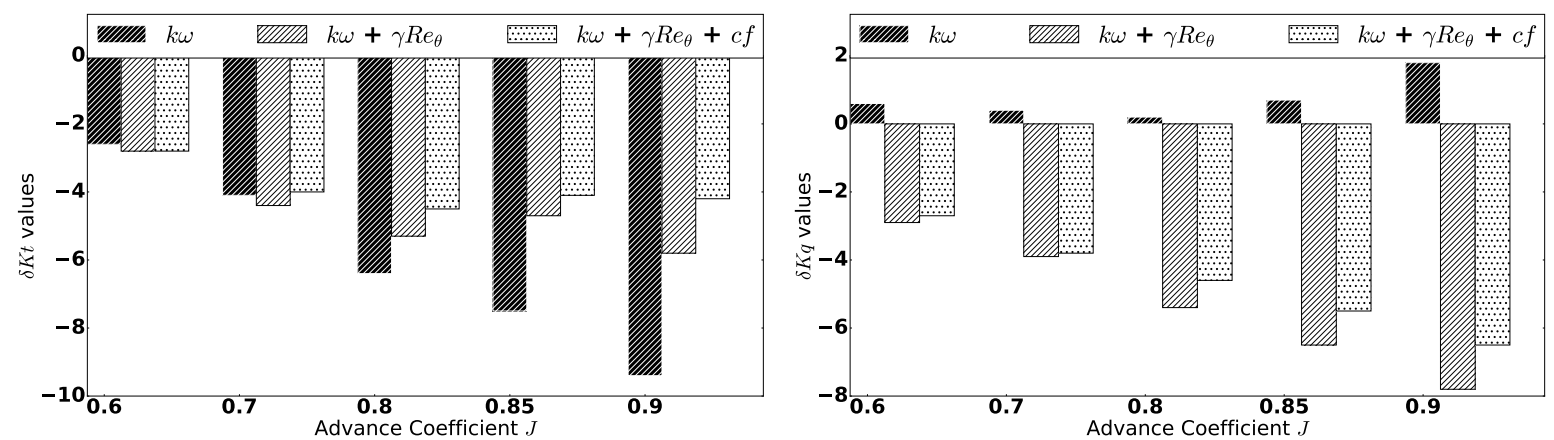

Figure 3.13: Differences in dimensionless thrust and torque respect to the experimental values for the CLT propeller.

ences change when the thrust coefficient is computed, where the transition models are more accurate. As efficiency is the ratio between those coefficients, where the $K_{t}$ is pondered with one order of magnitude more than that of $K_{q}$ (see Equation 3.1), the final efficiency tends to the experimental results when transition models are used, as Figure 3.12 shows.

Figure 3.14 clearly shows the differences between these models in terms of the averaged friction coefficient on different sections along the radius. Depending on the transition model, the transition onset moves appreciably, from $r / R \approx 0.85$ without cross-flow to $r / R \approx 0.75$ when the cross-flow modelization is applied. When the simulations with and without the cross-flow term are compared in terms of intermittency for the CLT propeller at $r / R=0.81$, relevant differences are found in the suction side, see Figure 
3.15.

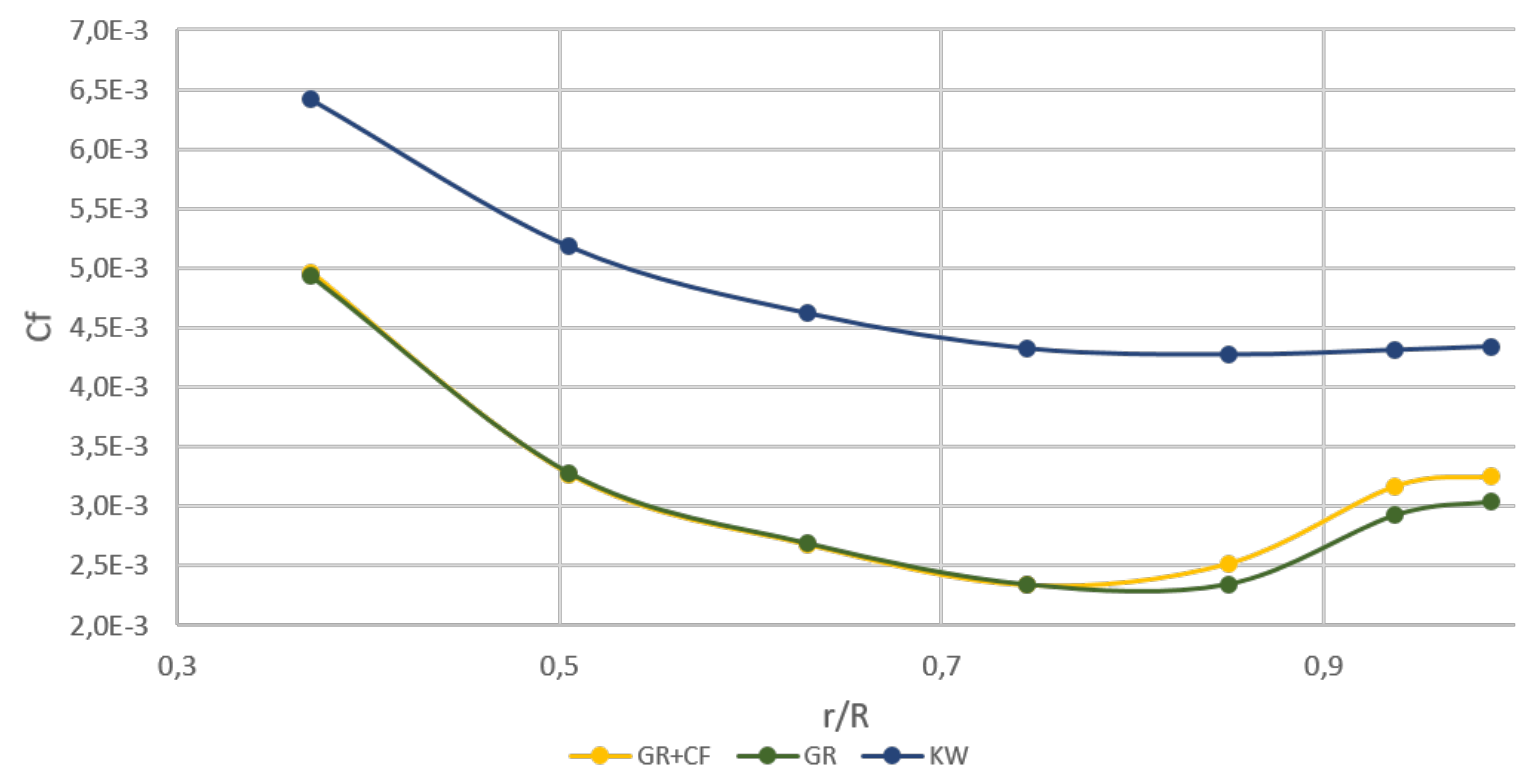

Figure 3.14: Skin friction comparison for different dimensionless radius for CLT propeller at design point $(J=0.8)$.

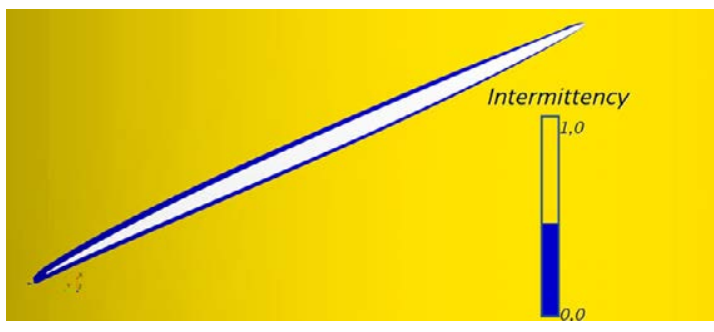

(a) $K \omega+\gamma R e_{\theta}$

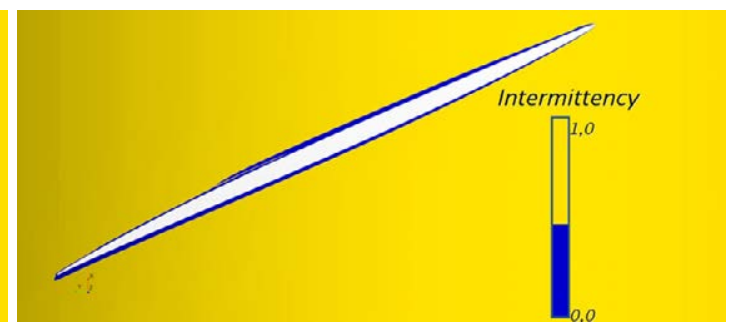

(b) $K \omega+\gamma \operatorname{Re}_{\theta}+$ crossflow

Figure 3.15: Intermittency scalar value for a cylindrical section of the CLT propeller at dimensionless radius $r / R=$ 0.81 and $J=0.8$.

In order to present a more complete description of the transition that takes place on the CLT propeller, Figure 3.16 shows the streamlines on both sides and a contour plot of the skin friction coefficient. As with the other two propellers, when a fully turbulent flow is assumed over the propeller's blade, the centrifugal component barely affects the slope of the streamlines, as can be observed when compared to the rest of the images where the transition model is included. Although the images for the $\gamma R e_{\theta}$ with and without cross-flow term seem to be very similar, an appreciable difference in the averaged skin friction coefficient can be found near the tip region for $r / R>0.75$, see Figure 3.14. 


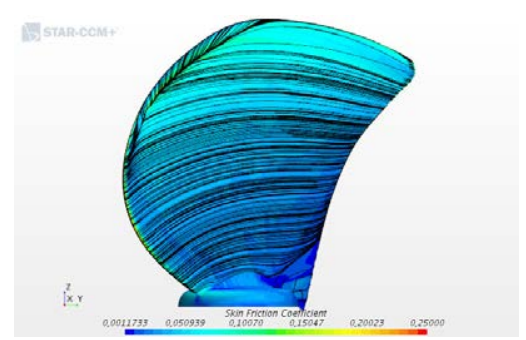

(a) $K \omega$

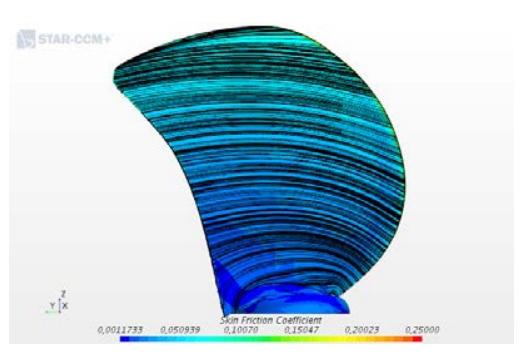

(d) $K \omega$

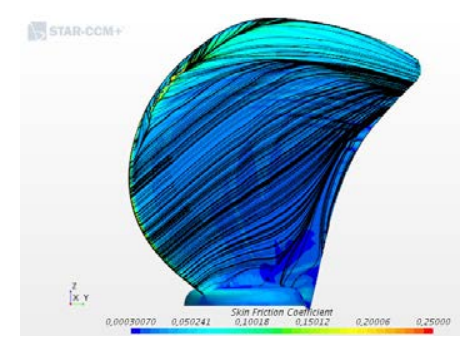

(b) $\omega+\gamma R e_{\theta}$

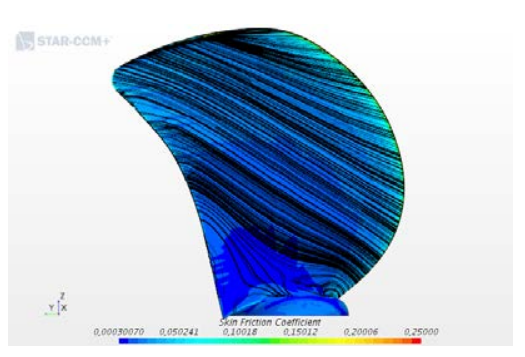

(e) $\omega+\gamma \operatorname{Re}_{\theta}$

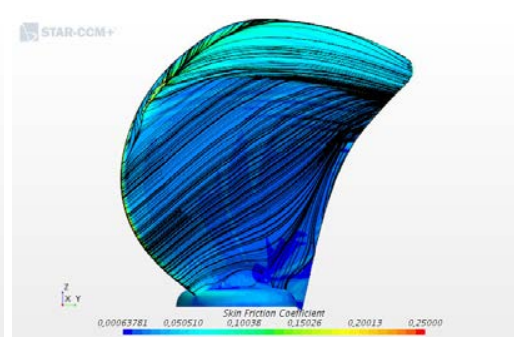

(c) $K \omega+\gamma R e_{\theta}+C F$

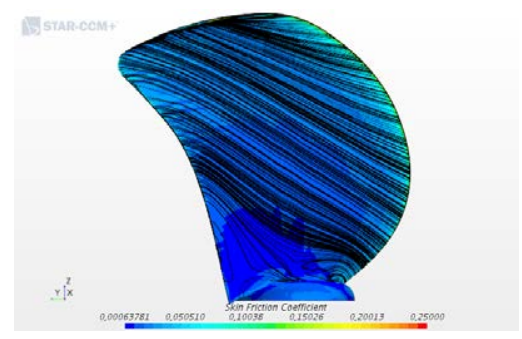

(f) $K \omega+\gamma R e_{\theta}+C F$

Figure 3.16: Skin friction contour plot and streamlines representation over the suction (top) and pressure (bottom) sides for the CLT propeller at $J=0.8$.

\subsubsection{VP1304 conventional Propeller}

In the following, results for the VP1304 propeller are presented. Analogously to the first case, this propeller was selected by the ITTC as a benchmark case, from which experimental measurements provided by the SVA Towing Tank are available for comparison with computational results. The range of advance coefficients studied are between $J=0.6$ and $J=1.4$, both included. For this case, the mesh has 9555805 cells and 20 prism layers in the boundary layer. Three different turbulence models were used, a fully turbulent $K \omega-S S T$ model, a $\gamma R e_{\theta}$ transition model that controls the regions where the $K \omega-S S T$ model is used and, lastly, a third variant where the transition model includes the modelization of the cross-flow transition mechanism.

In Figure 3.17, we can see the open water curves. The quantitative differences between each method compared to the experimental results obtained from the ITTC-Benchmark are quantified in Table 3.6. The errors compared to the experimental values are below five and four percent for the fully turbulent case and for the $\gamma R e_{\theta}$ transition model, respectively. It bears mentioning that when the cross-flow correlation is included in the $\gamma R e_{\theta}$ transition model, it predicts the experimental efficiency values very accurately for all advance ratios, being the difference below two percent in the worst case. We can conclude that the improvement obtained when the cross-flow term is added to the computational model is remarkable obtaining a good agreement with experimental values. 


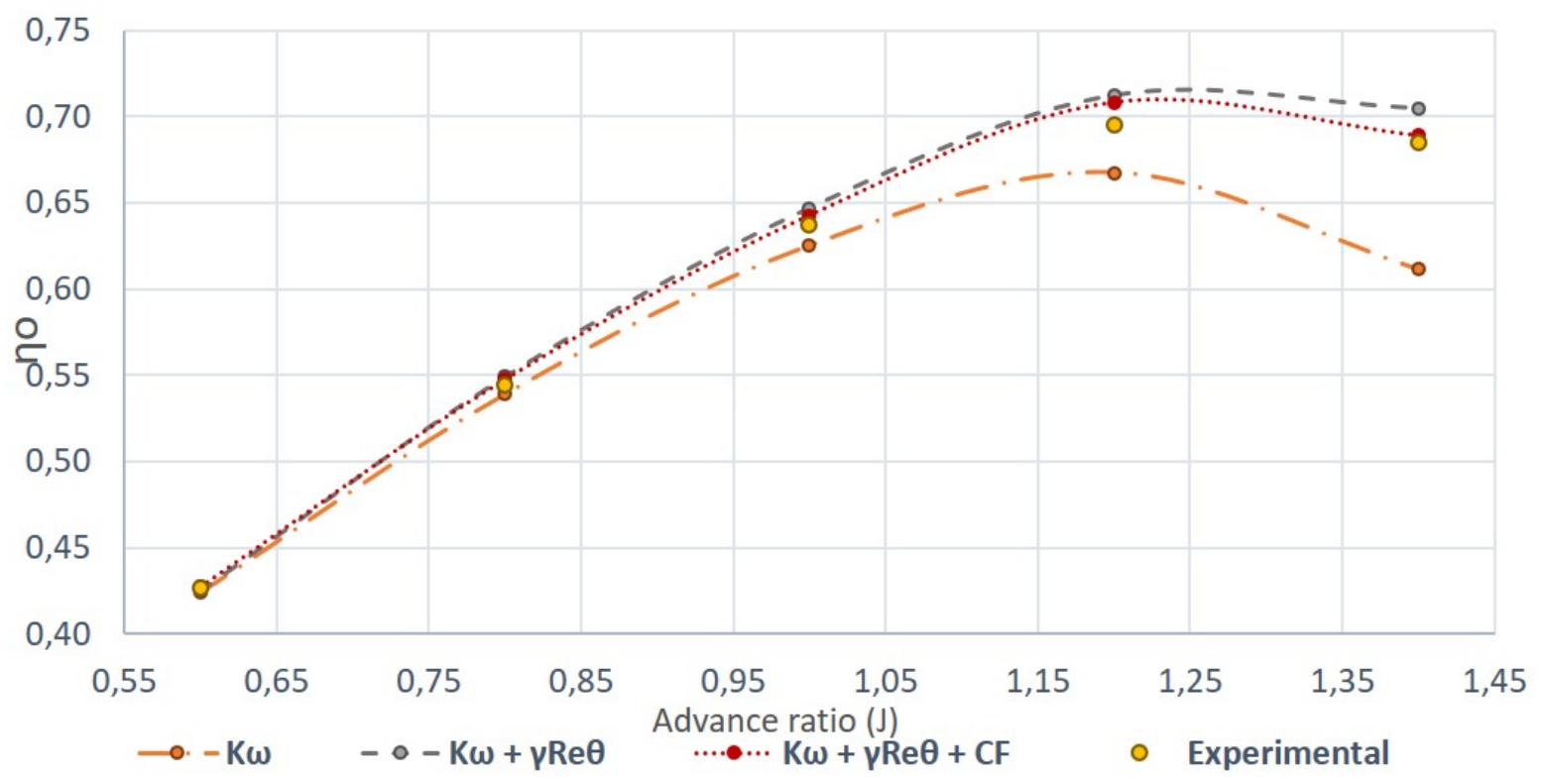

Figure 3.17: Efficiency values of the open water test for the VP1304 propeller.

\begin{tabular}{|c|c|c|c|}
\hline $\mathrm{J}$ & $K \omega-S S T \Delta_{\eta_{0}}[\%]$ & $\gamma R e_{\theta} \Delta_{\eta_{0}}[\%]$ & $\gamma R e_{\theta}+$ Cross - Flow $\Delta_{\eta_{0}}[\%]$ \\
\hline 0.6 & -0.72 & 0.91 & 0.02 \\
\hline 0.8 & -0.98 & 0.91 & 0.60 \\
\hline 1.0 & -1.88 & 1.59 & 0.90 \\
\hline 1.2 & -4.10 & 2.47 & 1.88 \\
\hline 1.4 & -10.70 & 2.95 & 0.64 \\
\hline
\end{tabular}

Table 3.6: Differences on the efficiency values respect to the experimental values for the VP1304 propeller.

In Figure 3.18, the prediction of the thrust and torque coefficient is quantified. We can observe that the $\gamma R e_{\theta}$ shows smaller differences in both the $K_{t}$ and $K_{q}$ coefficients than the fully turbulent model, although these results are slightly improved when the cross-flow term is added. The biggest differences with respect to the experimental values are found at $J=1.4$, a point which is far from the operative range of the propeller. Advance ratios that are situated on the decreasing part of the efficiency curve imply regions of the propeller working with negative angles of attack.

Figure 3.19 shows the differences between these models in terms of the average of the skin fric- 

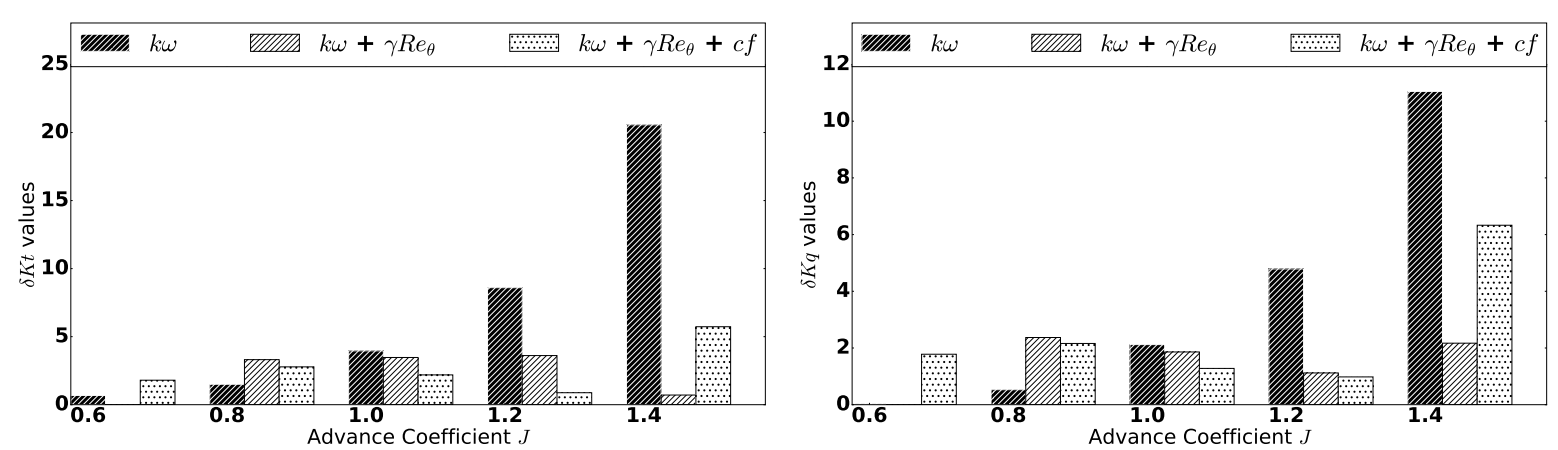

Figure 3.18: Differences in dimensionless thrust and torque with respect to the experimental values for the VP1304 propeller.

tion coefficient for the different sections considered along the radius. In this kind of representation we identify where the transition occurs by an increment in the skin friction coefficient. The growth observed at the tip region $(r / R>0.95)$ is not due to transition, and can be interpreted as a consequence of the tip vortex phenomenon. According to Figure 3.19, we could only observe the transition onset when the cross-flow term is applied to the model, and a relevant growth in the skin friction coefficient is observed at $r / R \sim 0.75$.

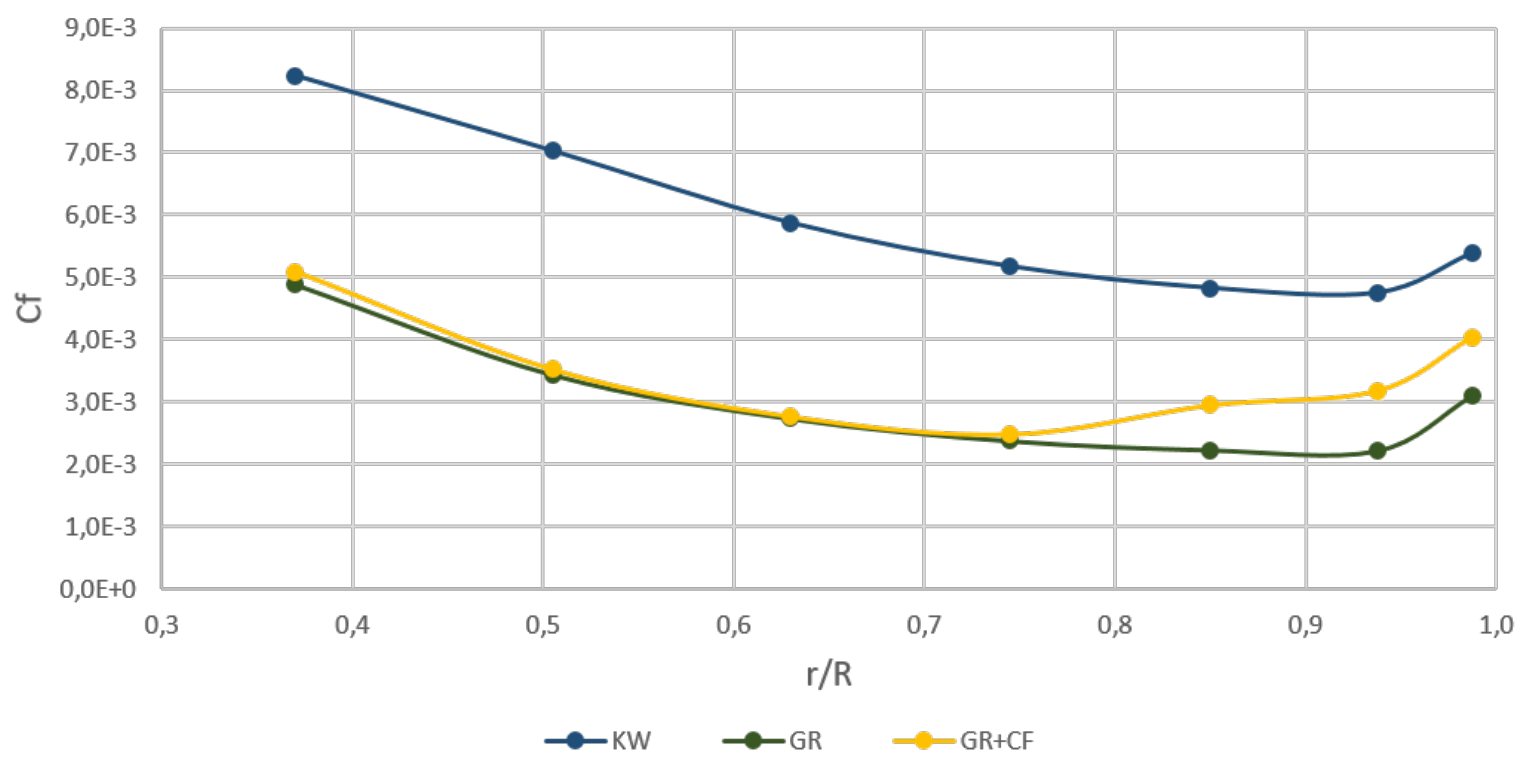

Figure 3.19: Skin friction comparison for different dimensionless radii for the VP1304 ITTC benchmark propeller.

In order to have a more complete description of the transition phenomenon on the tip region, the intermittency contours have been plotted at $r / R=0.9375$ in Figure 3.20, where turbulent flow can be identified by intermittency values of $\gamma \simeq 1$ and laminar flow of $\gamma \ll 1$. In this Figure, we can see that 


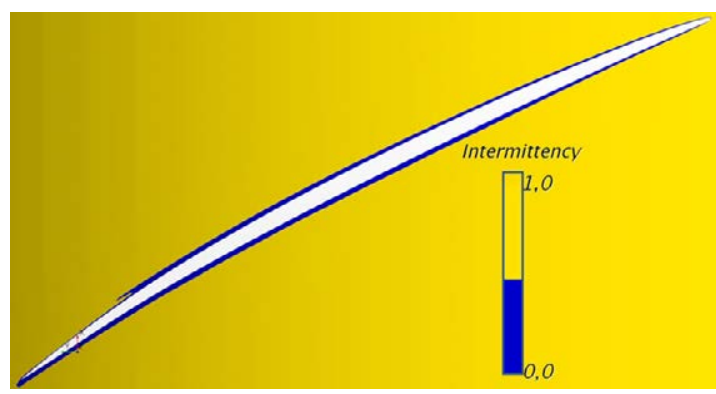

(a) $K \omega+\gamma R e_{\theta}$

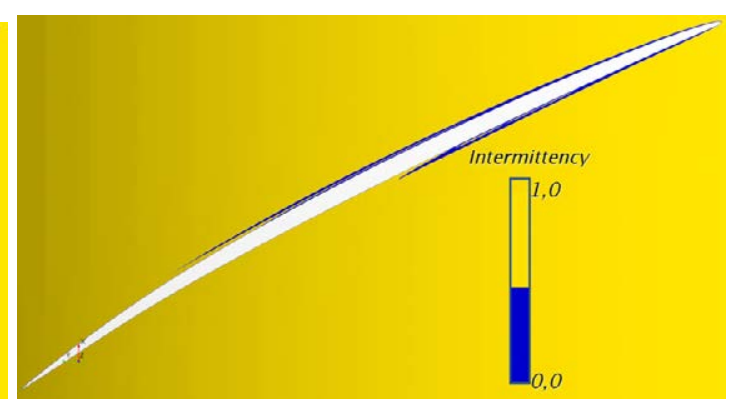

(b) $K \omega+\gamma \operatorname{Re}_{\theta}+$ crossflow

Figure 3.20: Intermittency scalar value for a cylindrical section of the VP1304 ITTC benchmark propeller at dimensionless radii $r / R=0.9375$ and $J=1.2$

when the simulations with and without the cross-flow correlation in the transition model are compared, the transition onset moves towards the leading edge on both the suction side and the pressure sides, being more intense and easier to appreciate on the pressure side. This behavior can be explained due to the fact that cross-flow is the most relevant transition cause on the pressure side, as highlighted by Dagenhart (1992).

Figure 3.21 shows the streamlines over suction and pressure sides for the three different models studied. The assumption of fully turbulent flow does not show the typical centrifugal component on the streamlines observed when a transition model is included. When the $\gamma-R e_{\theta}$ model is added to the simulation a larger centrifugal component appears in the laminar area on both the suction and pressure sides, while growing transition instabilities affect the streamlines at large radius near the trailing edge. If a cross-flow term completes the model, higher skin friction coefficients are appreciated in the transition area and, consequently, the direction of the streamlines changes more abruptly when the transition phenomenon starts.

\subsection{New friction line from CFD results}

All the methods presented at Subsection 2.1 have one thing in common, the corrections in $K_{t}$ and $K_{q}$ depend, with certain differences between them, on the difference between the drag coefficient $C_{D}$ at model and full scale, $\Delta C_{D}=C_{D, s}-C_{D, m}$. The drag coefficient is composed by the shear and pressure 


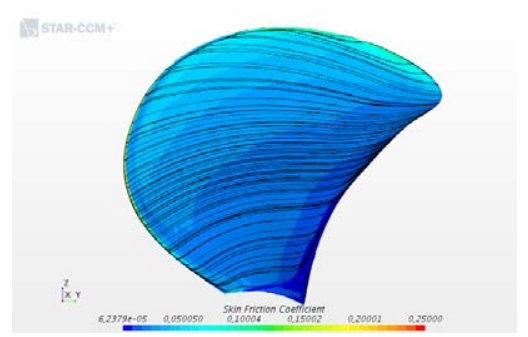

(a) $K \omega$

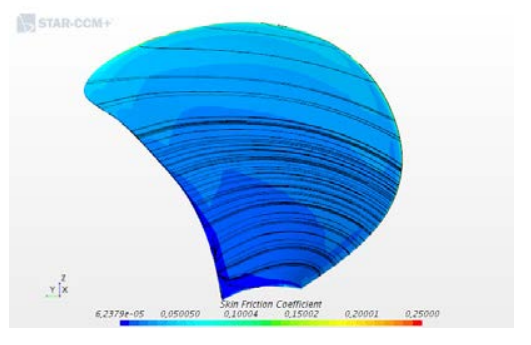

(d) $K \omega$

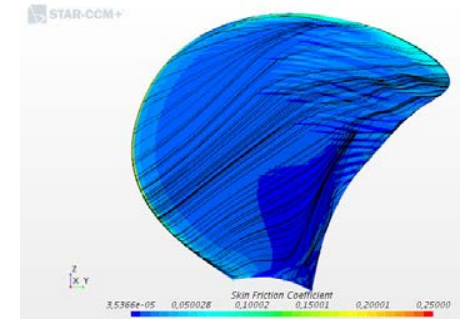

(b) $\omega+\gamma R e_{\theta}$

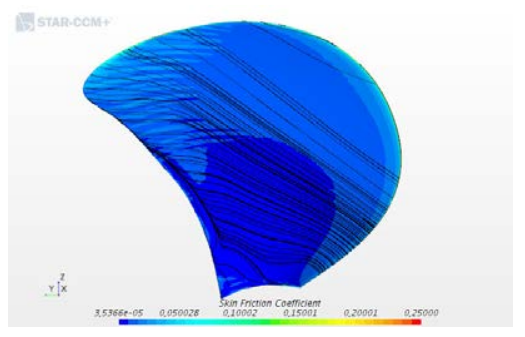

(e) $\omega+\gamma \operatorname{Re}_{\theta}$

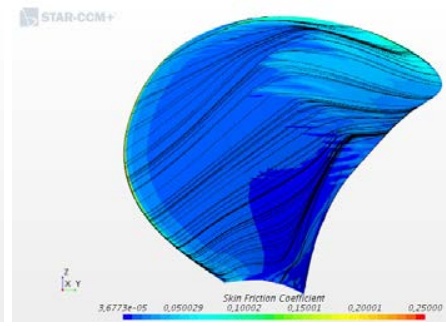

(c) $K \omega+\gamma R e_{\theta}+C F$

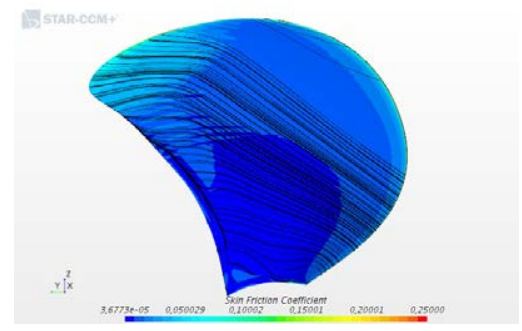

(f) $K \omega+\gamma R e_{\theta}+C F$

Figure 3.21: Skin friction contour plot and streamlines representation over the suction (top) and pressure (bottom) sides for the VP1304 ITTC Benchmark propeller at $J=1.2$.

forces and it is calculated applying a form factor ' $k$ ' to the shear component $C_{f}$, see Equation 3.2.

$$
\begin{aligned}
C_{D, m} & =k * C_{f, m} \\
C_{D, s} & =k * C_{f, s} \\
\Delta C_{D} & =k * \Delta C_{f}
\end{aligned}
$$

The methods presented in Subsection 2.1 imply differences on the estimation of the shear component of the drag coefficient, due to their particular friction line and differences in the estimation of the form factor ' $k$ ', presented by the Equation 3.3, for the particular ITTC - 78 and $S V A$ methods.

$$
\begin{aligned}
k_{I T T C 78} & =2 *(1+2 * T H C R) \\
k_{S V A} & =2 *\left(1+2.7 * T H C R+100 * T H C R^{4}\right)
\end{aligned}
$$

The laminar to turbulent transition may affect in great manner the friction coefficient $C_{f}$ estimation, however, still today there are uncertainties about when transition occurs at model scale due to the complexity of the physical phenomena. With this study, the author tries to shed some light on the subject based on CFD results. Furthermore, two new methods to estimate the friction coefficient are presented, which account for the laminar to turbulent transition effects.

In order to carry out this study, seven ship propeller geometries have been studied. The propellers 
descriptions are presented in the Appendix $A$ and the meshes for these propellers are based on the conclusions obtained for the mesh convergence process presented in the Appendix C. All the propellers have been radially sectioned in order to obtain the frictional forces acting on each section. Also, a systematic variation of the Re has been done in order to investigate the effects of the laminar to turbulent transition on the friction coefficient $C_{f}$. The main objective is to obtain the nose-tail friction coefficient $C_{f, n t}$ distribution which depends on the Re and other geometrical characteristics, see Equation 3.4, where $\tau$ is the wall shear stress, and $x$ is the dimensionless curvilinear coordinate along the chord section.

$$
C f_{n t}=\int_{0}^{1} \frac{\tau_{n t}(x)}{0.5 * \rho * V_{\infty}^{2}} d x=\int_{0}^{1} \frac{\tau(x) \cdot \overrightarrow{n t}}{0.5 * \rho * V_{\infty}^{2}} d x
$$

With the assumption that the $C_{f, n t}$ is equivalent to the $C_{f}$ of a flat plate, results obtained from CFD simulations and the rest of the friction lines can be plotted in the same graph.

In this study, there are two main differences to obtain the $C_{f}$ with respect to the study presented in Streckwall et al. (2013) and Bugalski et al. (2013). The first one is that only the nose-tail component of the shear forces over the section, $\tau_{n t}$, have been taken into account. This consideration has been assumed because the radial component of the wall shear stress does not affect the thrust and torque of the propeller section. The second one is that the transition model used includes cross-flow transition effects. All the simulations performed at model scale have been done considering transition effects using the model $\gamma-R e_{\Theta}+$ CrossFlow explained in Subsection 2.2. With respect to the full scale simulations, the fully turbulent approach $(K \omega-S S T)$ is considered for all the cases.

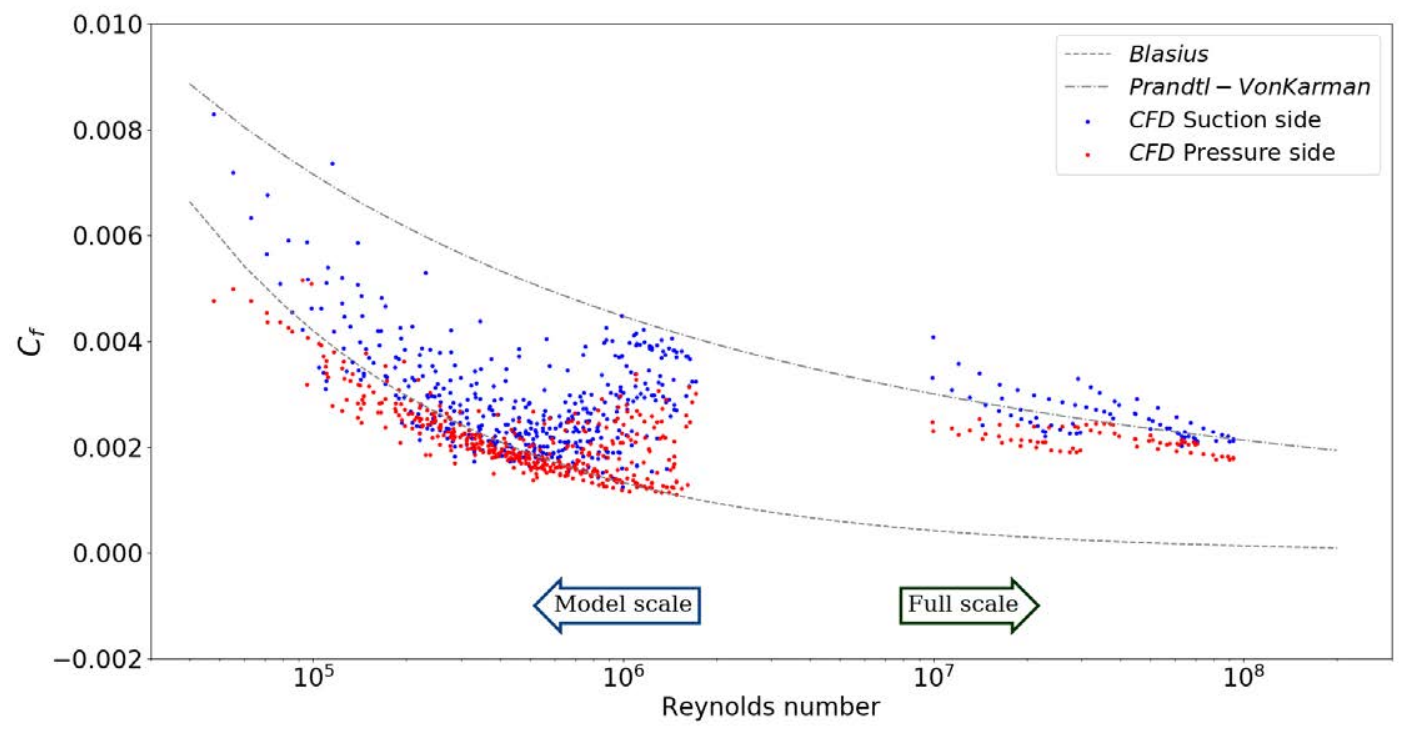

Figure 3.22: $C_{f}$ for all the sections studied separated in pressure and suction side. 
In Figure 3.22, results of the $C_{f}$ for each section of the seven propellers studied are presented. Results are divided on pressure and section sides. From the results, it is possible to appreciate a different behaviour of $C_{f, n t}$ between both sides. Suction side points present, in general, larger $C_{f, n t}$ than the pressure side points. This phenomenon is a consequence of a different transition onset and different velocities over the profile for each side.

With the objective of simplifying the final $C_{f}$ models, the difference between suction $\left(C_{f, s}\right)$ and pressure $\left(C_{f, p}\right)$ sides of the blade has not been taken into account, consequently the average value has been calculated as $C_{f}=\frac{C_{f, s}+C_{f, p}}{2}$. This average value is presented in Figure 3.23 for all the propellers of this study. In this Figure, colours represent the thickness-chord ratio (THCR) of each section. It is possible to appreciate that the lower is the THCR ratio the closer are the points to the theoretical lines. These trends are expected since the lower is the THCR coefficient the more similar is to a flat plate. The influence of this parameter will be taken into account in the new methods presented.

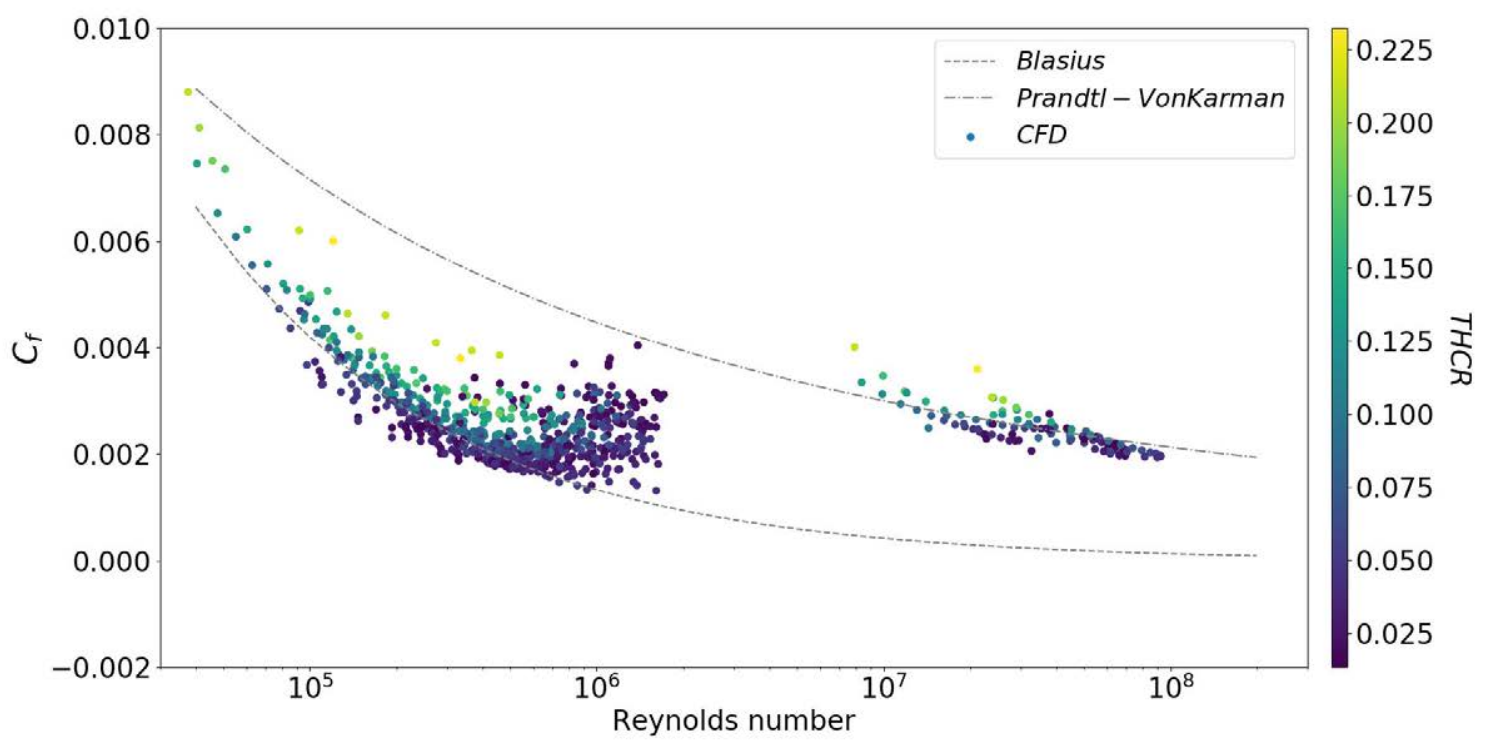

Figure 3.23: Dimensionless wall shear stress for all the propeller studied.

In Figure 3.24, different friction lines ${ }^{1}$ are presented and compared with the CFD results. The ITTC-78ITTC (2011) is the most common friction line for propeller extrapolation, the $S V A$ is a new line for the new method developed by SVA towing tank, see Klose et al. (2017). The original STRIPSIS, is a

\footnotetext{
${ }^{1}$ The methods to estimate the $C_{f}$ presented in ITTC (2011) will be named in the legend of the plots as $I T T C-78$, the one presented in Klose et al. (2017) as SVA and the original STRIPSIS presented in Perez-Sobrino et al. (2016) as $S T_{v o}$. The new method to estimate the $C_{f}$ based on the one presented in Perez-Sobrino et al. (2016) will be named as $S T_{v m}$ and the last one based on ANN as $M L P$.
} 
strip-based extrapolation method published in Perez-Sobrino et al. (2016), where the friction coefficients calculation depends on a certain Reynolds number range for laminar, turbulent and transitional areas of the blade. The $C_{f}$ of the laminar and turbulent areas are calculated with the Blasius and Prandtl-Von Karman friction lines respectively. For the case of the areas with mixed flow, the $C_{f}$ is calculated by interpolation between the Blasius and Prandt-Von Karman lines, where the input values of this calculations are the Reynolds number of each section $R e_{i}$. The last two methods try to become internationally standard recognized methods to extrapolate the propeller open water curves.

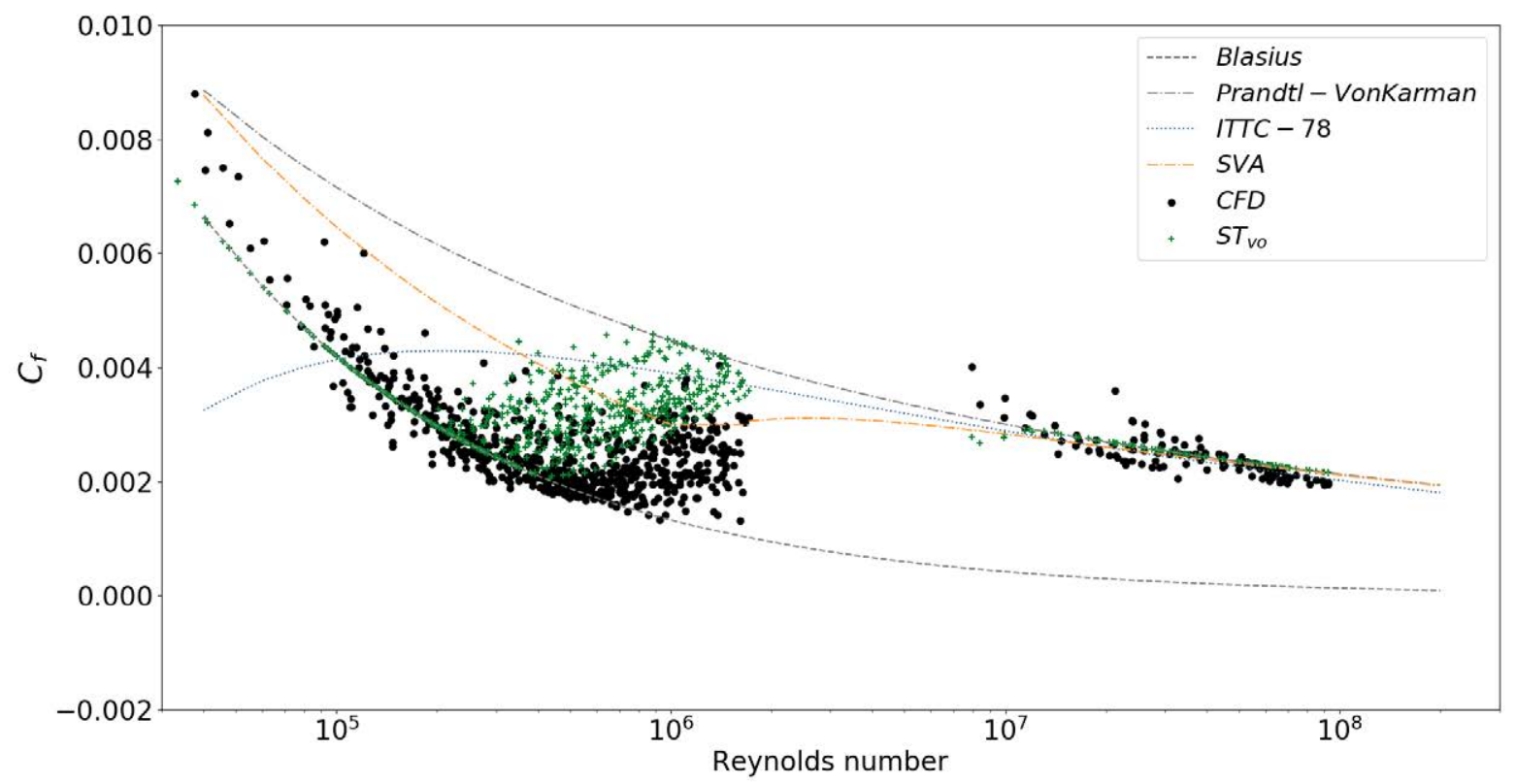

Figure 3.24: $C_{f}$ for each section studied compared with the most common friction lines.

As it is possible to appreciate in Figure 3.24, at model scale Reynolds range, the ITTC -78 friction line is qualitatively far away from the CFD results. The $S V A$ line is qualitatively closer than $I T T C-78$ but for low Re is not very accurate when compared to CFD results. The behaviour of the original STRIPSIS method is the best approximation to the dataset of the CFD propeller simulations. For the full scale Reynolds range all the methods are qualitatively and quantitatively close to the CFD results.

With the aim to improve the $C_{f}$ estimation of a certain section of the propeller, two new methods to estimate the $C_{f}$ have been created and the results are presented in Figure 3.25. The first one is an improvement of the original STRIPSIS with a reconsideration of the critical Reynolds number, at which the flow becomes turbulent at model scale, and also considering that the flow at full scale is always turbulent, details may be found in Appendix D.2. The name of this new model is STRIPSIS modified. For the development of the second method, a MLP has been trained with all the dataset presented in 


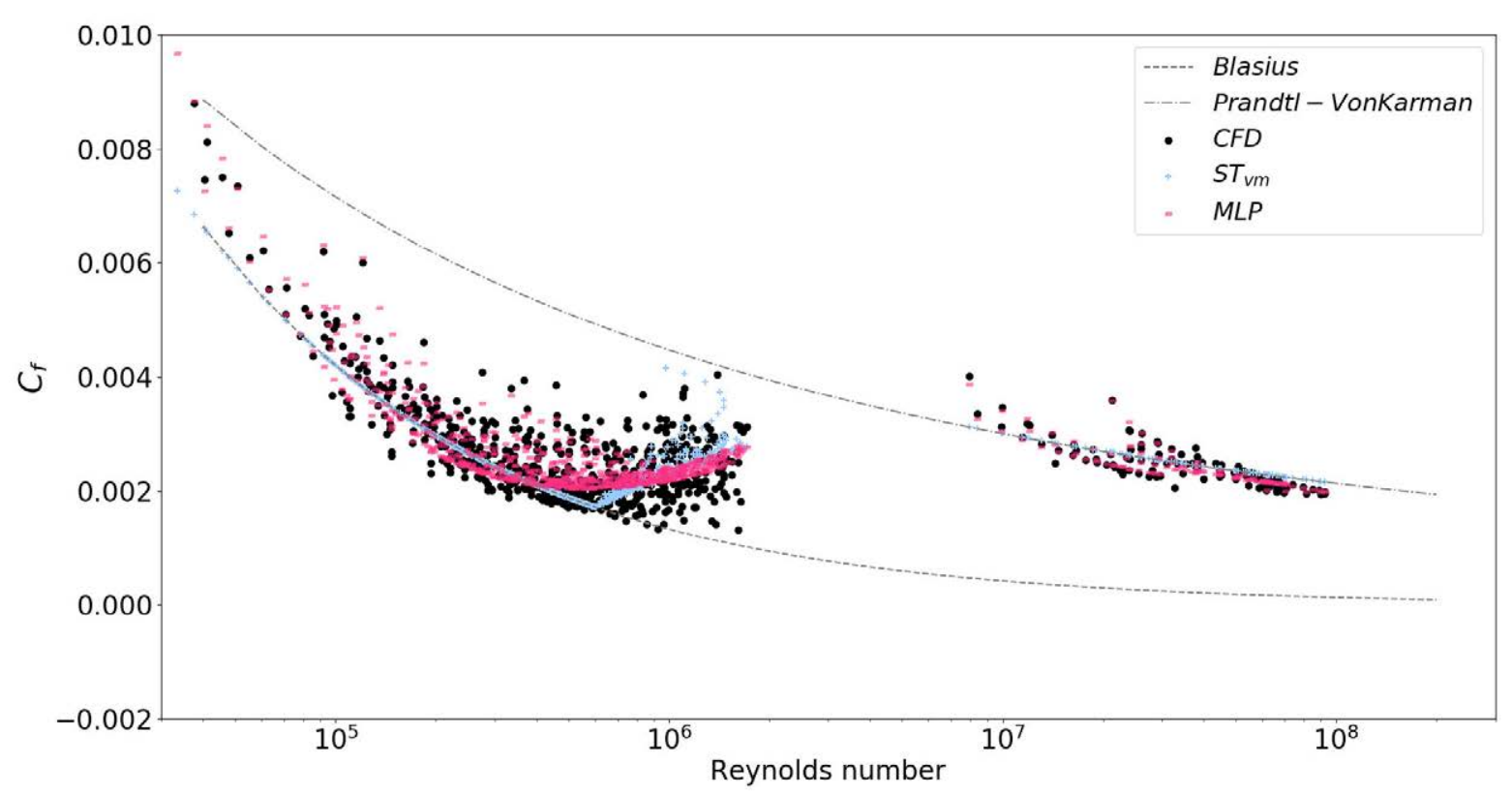

Figure 3.25: $C_{f}$ for each section studied compared with the two new methods presented, STRIPSIS $S_{\text {modified }}$ and $M L P$. Blasius and Prandt-Von Karman lines are reference lines.

this section. It is an ANN-based method that takes into account geometrical parameters of the section and the Re. The details of the MLPs used in this section are presented in Appendix D.2. It is possible to appreciate a good behaviour for both methods, with higher dispersion of the MLP for $R e \leq 7 \cdot 10^{5}$, from this Reynolds number and for model scale, the MLP method is slightly saturated. This is due, to the fully laminar assumption in a certain range of the modified STRIPSIS, and due to the geometrical component in the MLP method.

As it may be deducted from Equation 3.2, the most important parameter to estimate the drag scale effects between model and full scale is the difference between model and full scale friction forces. A comparison of this parameter $\Delta C_{f}$, for each one of the sections studied, is presented in Figure 3.26. It is possible to appreciate that the trend of the $I T T C-78$ method is totally different, when compared with the rest of the methods, and its tendency is to under-predict the $\Delta C_{f}$ for $R e \leq 2 \cdot 10^{5}$. It is important to note that this is the minimum Reynolds number to carry out the OWT recommended by the ITTC committee. The $S V A$ method is the most optimistic for $R e \leq 3 \cdot 10^{5}$, while for higher Reynolds numbers this position is stolen by the $S T_{v}$. In general, the new methods, $S T_{m v}$ and $M L P$, present the same trends as the CFD results where a local maximum around $R e \approx 6 \cdot 10^{6}$ has been found.

Taking the liberty of assuming CFD results as the most realistic, a new parameter is defined $\left(\Delta C_{f}^{d i f f}=\right.$ $\Delta C_{f}^{\text {method,i }}-\Delta C_{f}^{C F D}$ ). This parameter has been created in order to quantify the accuracy of each 


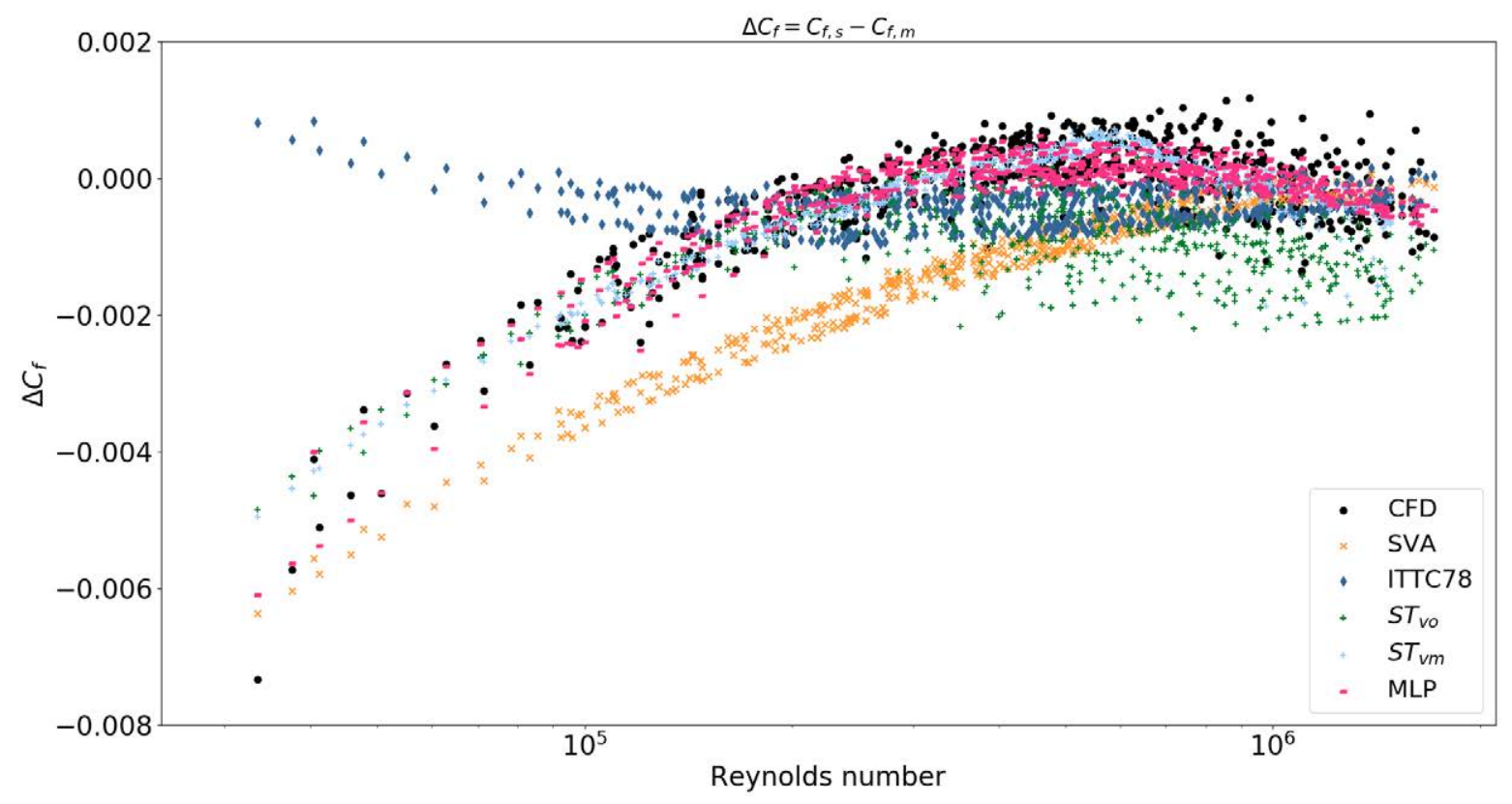

Figure 3.26: Difference between the full and model scale friction coefficients $\Delta C_{f}$ for all the methods presented at this section.

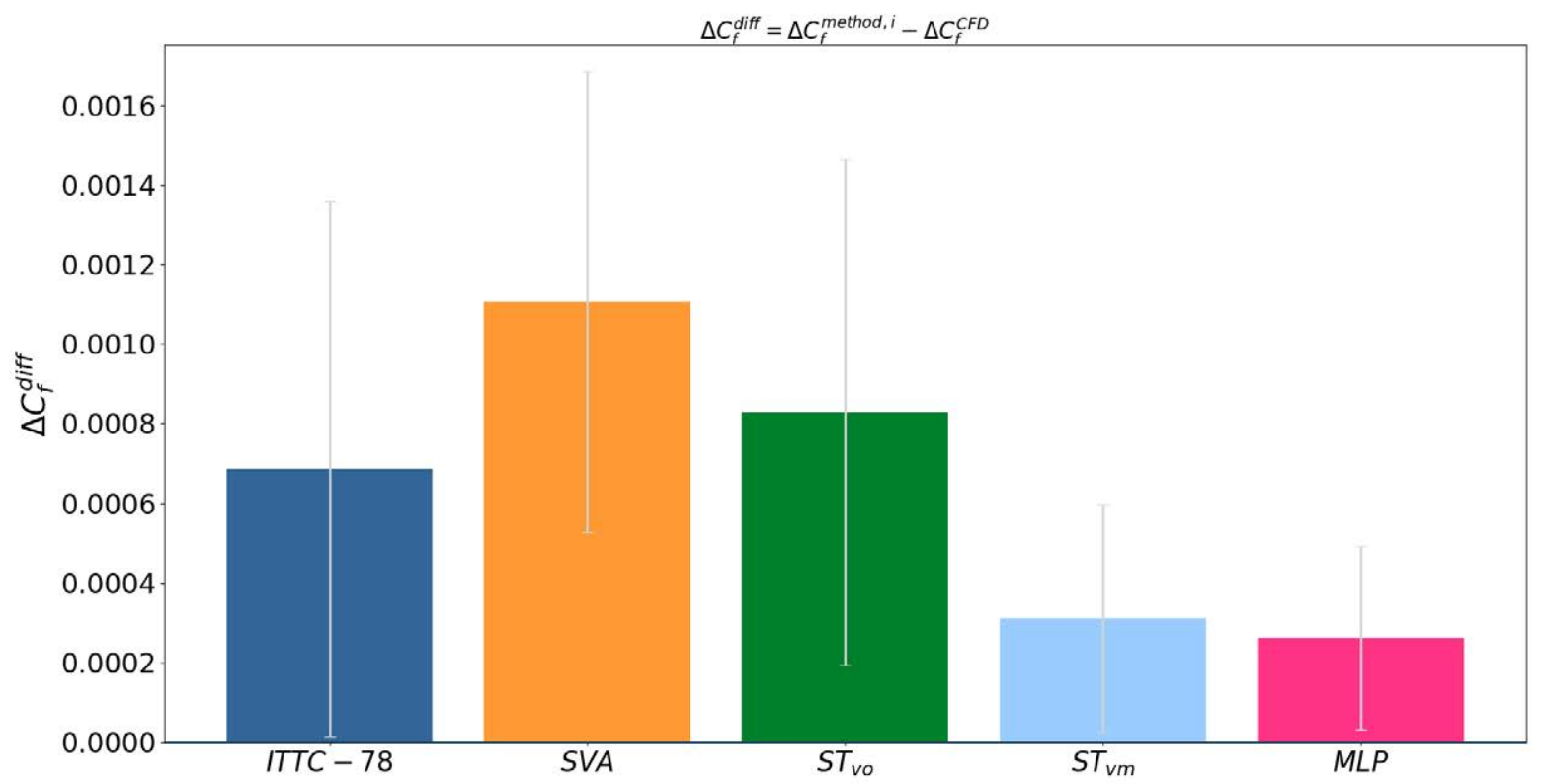

Figure 3.27: Mean and standard deviation of the difference between the $\Delta C_{f}$ for all the points of of each method $\left(\Delta C_{f}^{\text {method,i }}\right)$ and $\Delta C_{f}$ obtained with $C F D$ results.

method. In Figure 3.27, the mean values and the standard deviation of $\Delta C_{f}^{d i f f}$ are presented. It is possible to appreciate that the new methods presented have the best behaviour in terms of accuracy and standard deviation with respect to the CFD simulations. 


\subsection{New Form factor and lift scale effects}

In the following, a complete analysis of the sections of the CONV02 propeller has been done. The main objective is to estimate the viscous drag of the sections and the lift scale effects obtained with CFD. This propeller has been selected because it is the only one that is not contained in the training and testing dataset of the Artificial Neural Network developed in the Appendix D.

The main motivation to propose a new form factor, is that when traditional methods are applied to obtain the drag coefficient of the sections, the results are considerably higher than the results obtained directly with CFD methods. The new form factor should be used with the friction coefficient obtained directly with CFD methods, or with any other method that simulates this as, for example, MLP. This happens because the friction coefficient obtained by CFD methods, a component of the traditional form factor estimation is implicitly considered.

Regarding the motivation to calculate the lift scale effects, when only scale effects due to the drag component are applied, is that the final corrections in thrust and torque coefficient still need an improvement to match the CFD results, this is going to be shown in Section 3.5. This improvement should be quantitative and qualitative.

\subsubsection{Identification of the AoA of the sections, $\alpha_{\beta_{i}}$}

Figure 3.28 presents an scheme of the velocity field and forces acting on a marine propeller blade section.

The first step, in order to estimate the drag and lift coefficients directly from CFD results, is to calculate the real incidence angle of the flow at each section, $\alpha_{\beta_{i}}$. This is because the lift, by definition, is perpendicular to the incident flow and the drag is parallel. In marine propellers, due to the induced velocities $\left(u_{a}\right.$ and $u_{t}$ ), the real angle of the flow is not known and it is usually assumed that the incidence flow has the nose-tail direction $(\overrightarrow{n t})$. Although the previous assumption is not far away from the reality, being marine propellers usually designed to work at $A o A=0^{\circ}$, in the following a procedure that computes the real angle of attack of each section has been considered. 


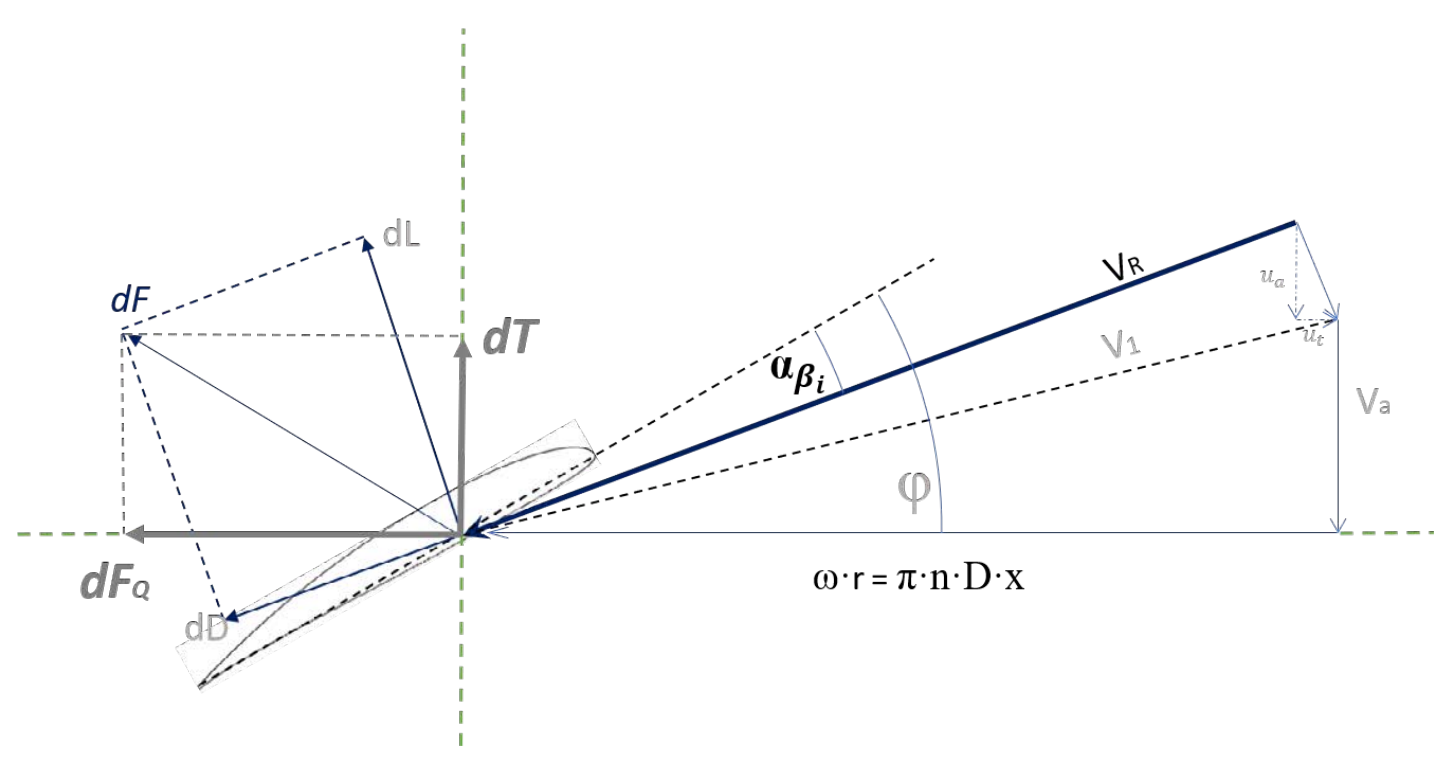

Figure 3.28: Marine propeller blade profile forces and velocities scheme.

Equation 3.5 represents the decomposition of the lift coefficient $C_{L}$ coefficient in the friction $\left(C_{L}^{f}\right)$ and pressure $\left(C_{L}^{p}\right)$ components projected to the incident flow direction.

$$
C_{L}=\int_{0}^{1}\left(C_{L}^{p}+C_{L}^{f}\right) d x \cdot \cos \left(\alpha_{\beta_{i}}\right) \quad C_{D}=\int_{0}^{1}\left(C_{D}^{p}+C_{D}^{f}\right) d x \cdot \cos \left(\alpha_{\beta_{i}}\right)
$$

The frictional forces are orders of magnitude lower than the pressure forces in the $C_{L}$ coefficients, consequently, it is possible to simplify Equation 3.5, assuming that the $C_{L}$ only depends on the pressure forces, see 3.6. Under this assumption, the flow incidence angle will be defined as the angle that maximizes the $C_{\text {Lift }}$.

$$
C_{\text {Lift }}=\int_{0}^{1} C_{L}^{p} d x \cdot \cos \left(\alpha_{\beta_{i}}\right)
$$

In Figures 3.29 and 3.30, the variation of the $C_{\text {Lift }}$ with respect to the projection angle or a pseudo Angle of Attack $\left(A o A^{\prime}\right)$ are represented for full and model scale. It is possible to appreciate the differences in the angle that satisfies the maximum lift condition in each case ${ }^{2}$.

Figure 3.31 shows the variation of $\alpha_{\beta_{i}}$ along the radii of the propeller. It is important to note that for lower sections, $r / R<0.5$, negatives $\alpha_{\beta_{i}}$ are obtained at model scale, consequently, the profile sections at these radii are working with negatives angles of attack. The smallest dispersion of the $\alpha_{\beta_{i}}$ is found

\footnotetext{
${ }^{2}$ It is important to note that positives $A o A^{\prime}$ mean negatives AoA of the section, satisfying the Equation $\left.A o A^{\prime}\right|_{\max \left(C_{\text {Lift }}\right)} *-1=$ $\alpha_{\beta_{i}}$.
} 


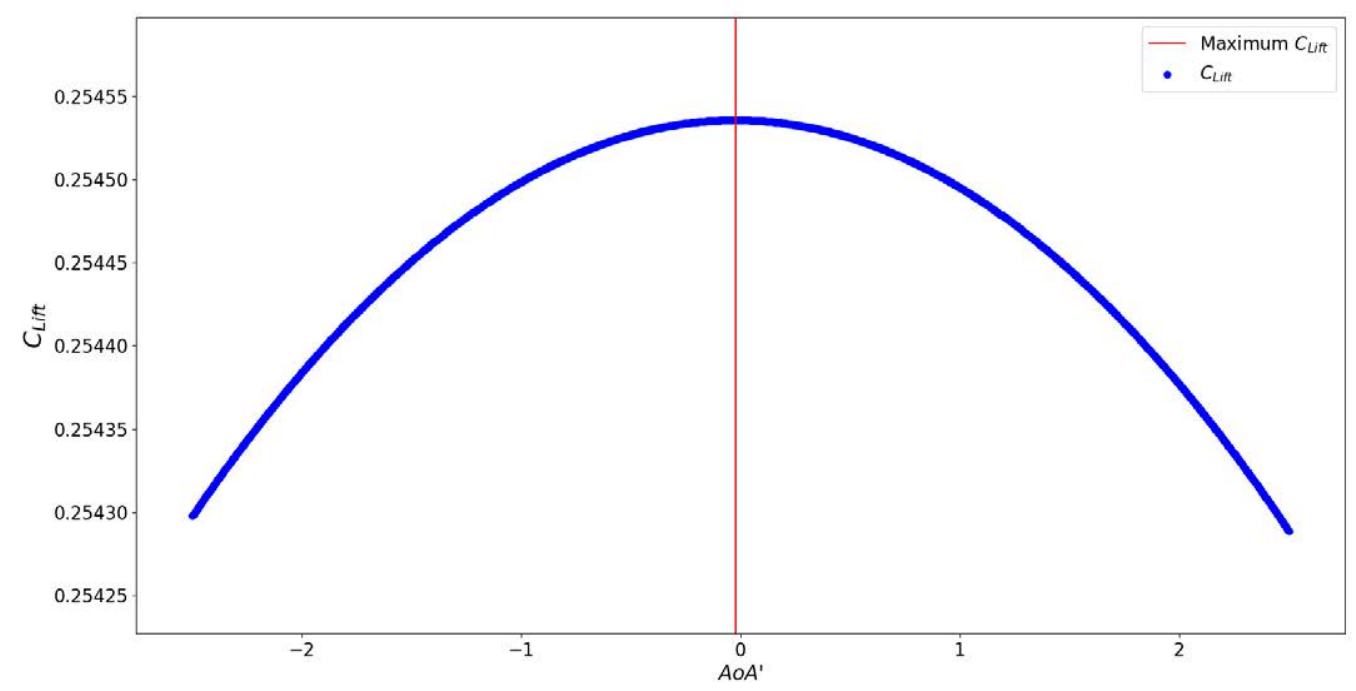

Figure 3.29: Lift coefficient for different projection angles. The angle that satisfies $\max \left(C_{L i f t}\right)$ is represented by a red line. Full scale simulation - radius $0.35 \mathrm{R}-5$ rps.

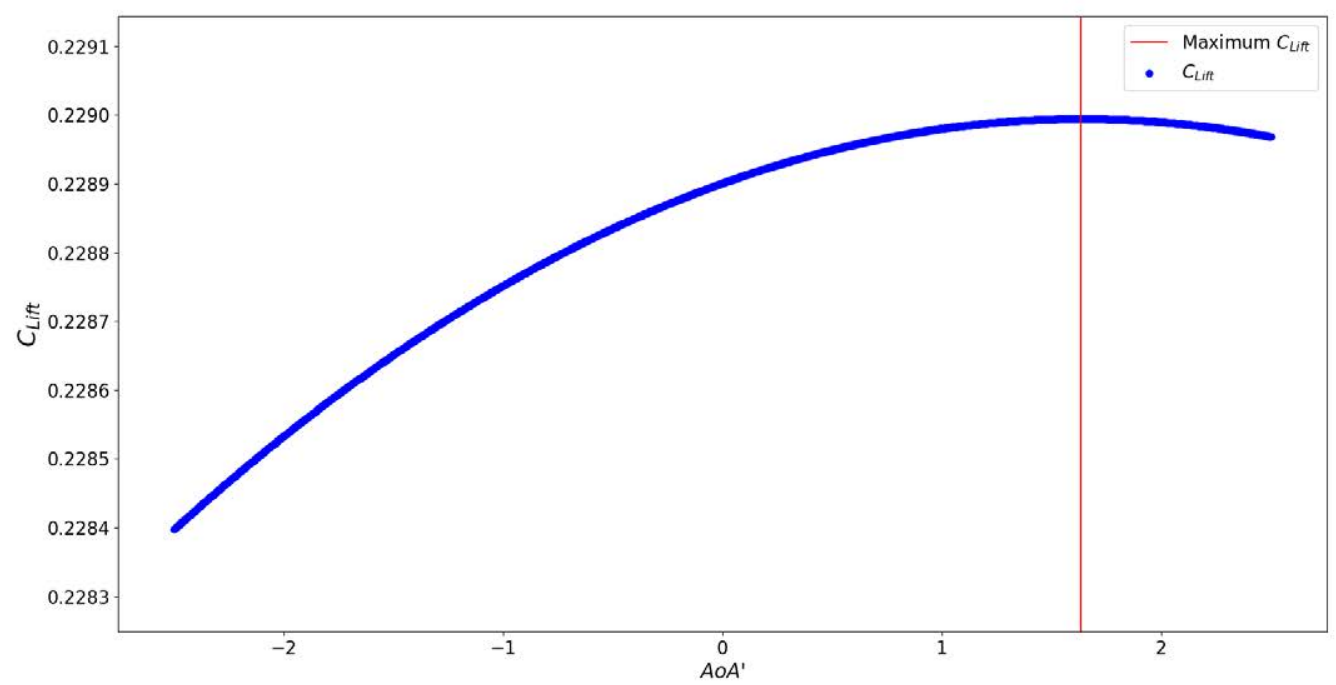

Figure 3.30: Lift coefficient for different projection angles. The angle that satisfies $\max \left(C_{L i f t}\right)$ is represented by a red line. Model scale simulation - radius $0.35 \mathrm{R}-5 \mathrm{rps}$.

at $r / R=0.7$, where the $A o A \approx 1^{\circ}$. Note that, at the tip region, the dispersion tends to increase. The phenomenon that occurs at lower radii can lead to high lift scale effect at these sections.

Once the $\alpha_{\beta_{i}}$ has been calculated applying the maximum lift condition for different projection di- 


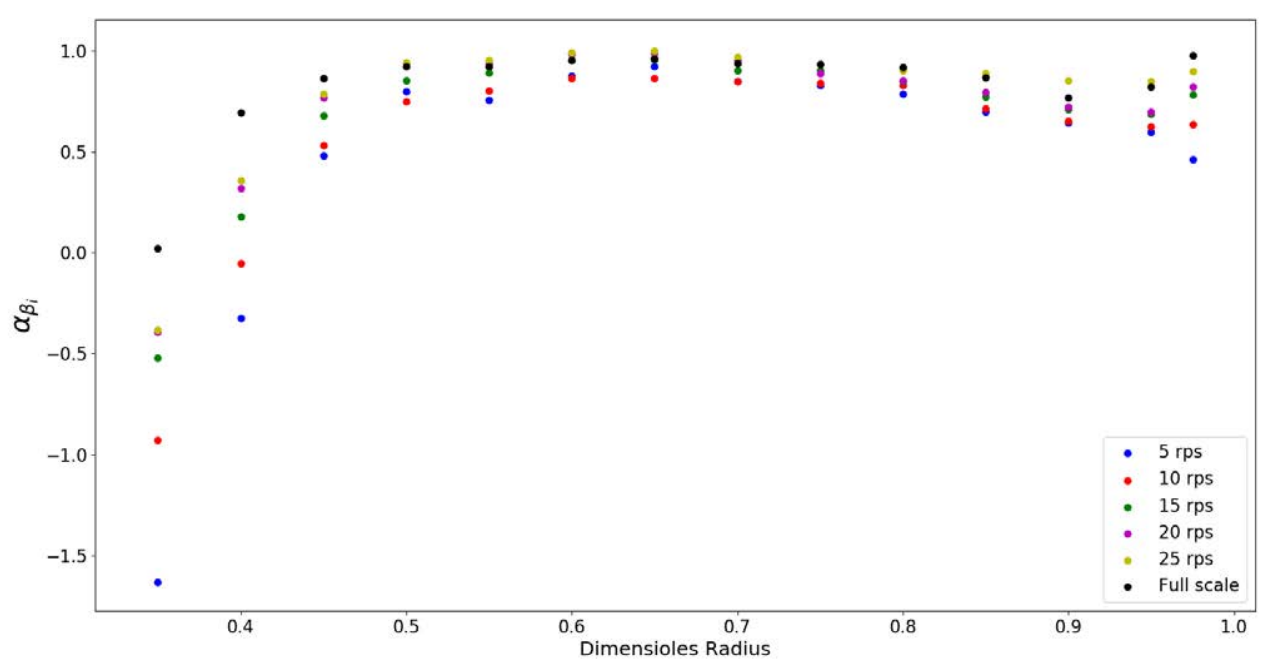

Figure 3.31: Angle of the incident flow $\alpha_{\beta_{i}}$ along the radii for different Reynolds of the $O W T$ simulations. Results for different rotating velocities at model scale and full scale are represented.

rections, it is possible to calculate the drag coefficient at each section and compare it with the results obtained with the different formulas available in the literature. Also, it will be very interesting to quantify the scale effects in $K_{t}$ and $K_{q}$ due to the lift forces.

\subsubsection{Form factor modification}

Usually, as it has been already explained, the drag coefficient $C_{D}$ is calculated applying a form factor to the friction coefficient $C_{f}$, see Equation 3.2. In this particular case, a comparison between the $C_{D}^{C F D}$ and the $C_{D}$ is obtained applying a form factor to the $C_{f}$ calculated by different methods (including the $C_{f}$ from CFD results), is going to be presented. One of the objectives of this analysis, among others, is to calculate a new form factor estimation for the MLP method, presented in Subsection 3.3. For the MLP method, a new form factor estimation is demanded because the $C_{f}$ obtained with this method includes a part of the conventional form factors that should be applied only to 'flat plate' friction coefficients.

In order to perform a better approximation of the drag coefficient obtained from the MLP method, it is necessary to modify the form factor estimation presented by the Equation 3.3. A new formula for the form factor estimation of the MLP method is presented in Equation 3.7. Figure 3.32, presents a comparison of the $C_{D}$ obtained with the form factor from Equations 3.3 and 3.7 and the one obtained directly with CFD. 


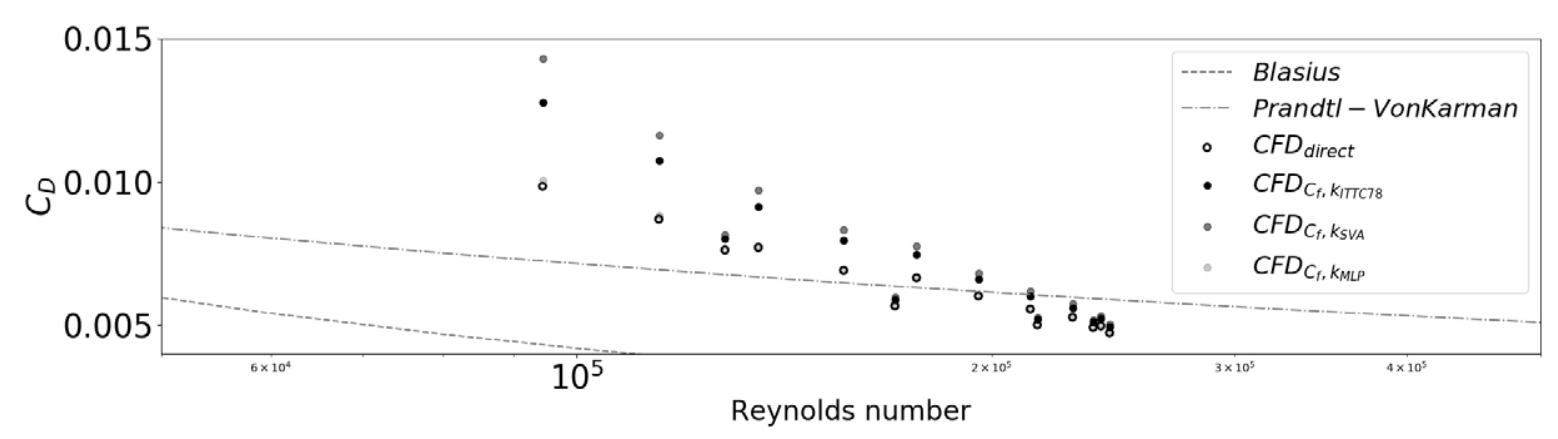

Figure 3.32: CONV02 sections comparison for different ways to estimate the $C_{D}$. The case represented is the $O W T$ simulation for model scale at $r p s=5$. Blasius and Prandtl lines are plotted only as reference values.

$$
k_{M L P}=2 *\left(1+T H C R^{2}\right)
$$

Under the assumption that the most accurate values are those obtained directly from CFD results, in Figure 3.33, the accuracy of each method respect to those extracted directly with CFD method is presented. This approximation is computed taking the average of the absolute differences obtained by comparison with the CFD results for all the Reynolds range. It is very interesting to observe how with the new formula presented in the Equation 3.7, the differences are below $0.5 \%{ }^{3}$ in contrast to the other methods where differences are above $9 \%^{4}$. The consequence is that when the friction coefficient is estimated with the MLP method, trying to match those obtained with CFD results, it is necessary to apply the form factor correction given by the Equation 3.7.

\subsubsection{Lift scale effects}

Lift scale effects are not usually taken into account because they are normally considered negligible and very difficult to measure, in a experimental campaign at model scale. Once the angle of incidence of the flow and all the forces over the profile are known, it is possible to know the lift of each section of the propeller blade. In Figure 3.31, it may be observed that, for low radii, the sections are working in negative

\footnotetext{
${ }^{3}$ The differences obtained in this part are based on friction coefficients extracted directly from CFD results, when the new form factor $k_{M L P}$ is applied to the friction coefficients calculated with the MLP method, presented in 3.3. Differences probably will be larger but due to the accuracy of the MLP and not due to the form factor calculation.

${ }^{4}$ If the form factors $k_{I T T C 78}$ and $k_{S V A}$ are applied to the skin frictions calculated by the ITTC -78 and $S V A$ methods, presented in 3.3, differences will be probably even smaller.
} 


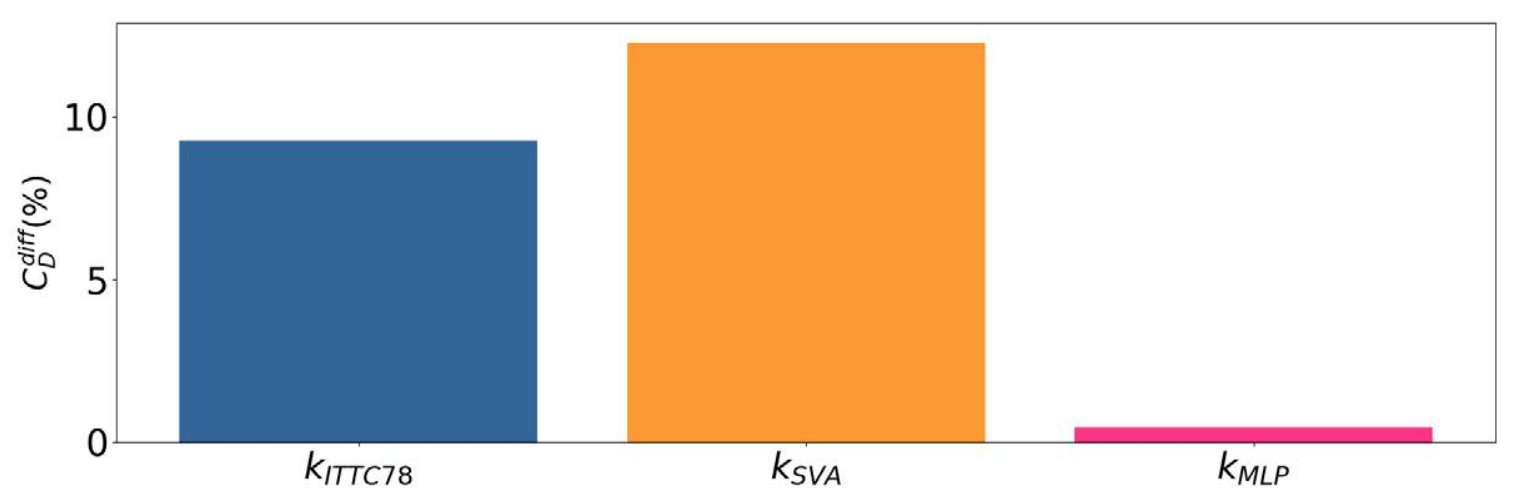

Figure 3.33: CONV02 propeller $C_{D}^{\text {diff }}(\%)$ mean values for all the range studied, using different form factor estimations.

angles of attack, the more negative angle the smaller is the Reynolds number. In this Subsection, the influence of the lift variation with the Reynolds number and its effects on the $K_{t}$ and $K_{q}$ coefficients is presented.

Considering the lift scale effects in $K_{t}$ and $K_{q}$ as those originated only by lift, Equation 3.8 presents the way to obtain the correction factors in $K_{t}$ from the pressure coefficient $C_{p}$.

$$
\begin{aligned}
\Delta K_{T, \text { lift }} & =K_{T, \text { lift }}^{s}-K_{T, \text { lift }}^{m}=\left.\frac{T_{\text {lift }}}{0.5 * \rho * n^{2} * D^{4}}\right|_{s}-\left.\frac{T_{\text {lift }}}{0.5 * \rho * n^{2} * D^{4}}\right|_{m}= \\
& =\left.\frac{C_{P} * C r * V_{0.7 R}^{2} * \cos \left(\alpha_{\beta_{i}}+\phi\right)}{n^{2} * D^{4}}\right|_{s}-\left.\frac{C_{P} * C r * V_{0.7 R}^{2} * \cos \left(\alpha_{\beta_{i}}+\phi\right)}{n^{2} * D^{4}}\right|_{m}
\end{aligned}
$$

Following the same procedure for the thrust coefficient, Equation 3.9 may be derived.

$$
\Delta K_{Q, l i f t}=\left.\frac{C_{P} * C r * V_{0.7 R}^{2} * \sin \left(\alpha_{\beta_{i}}+\phi\right) * r_{i}}{n^{2} * D^{5}}\right|_{s}-\left.\frac{C_{P} * C r * V_{0.7 R}^{2} * \cos \left(\alpha_{\beta_{i}}+\phi\right) * r_{i}}{n^{2} * D^{5}}\right|_{m}
$$

Figure 3.34 represents the $\Delta K_{t, i}$ and $\Delta K_{q, i}$ corrections due to lift scale effects along the radii for the different OWT simulations of the CONV02 propeller. This plot is very interesting because shows a very different, but coherent, behaviour for each case. It seems that there are two pivoting points that can be identified by a local maximum and a local minimum, where the local minimum is associated to the lower radii and the local maximum to the upper radii. These characteristic points change with the rotating velocity, consequently with the Reynolds number, varying the local minimum probably due to the laminar separation and being the local maximum more related with the laminar to turbulent transition.

The existence of the two pivoting points leads to a very non-linear behaviour in the lift scale effects 

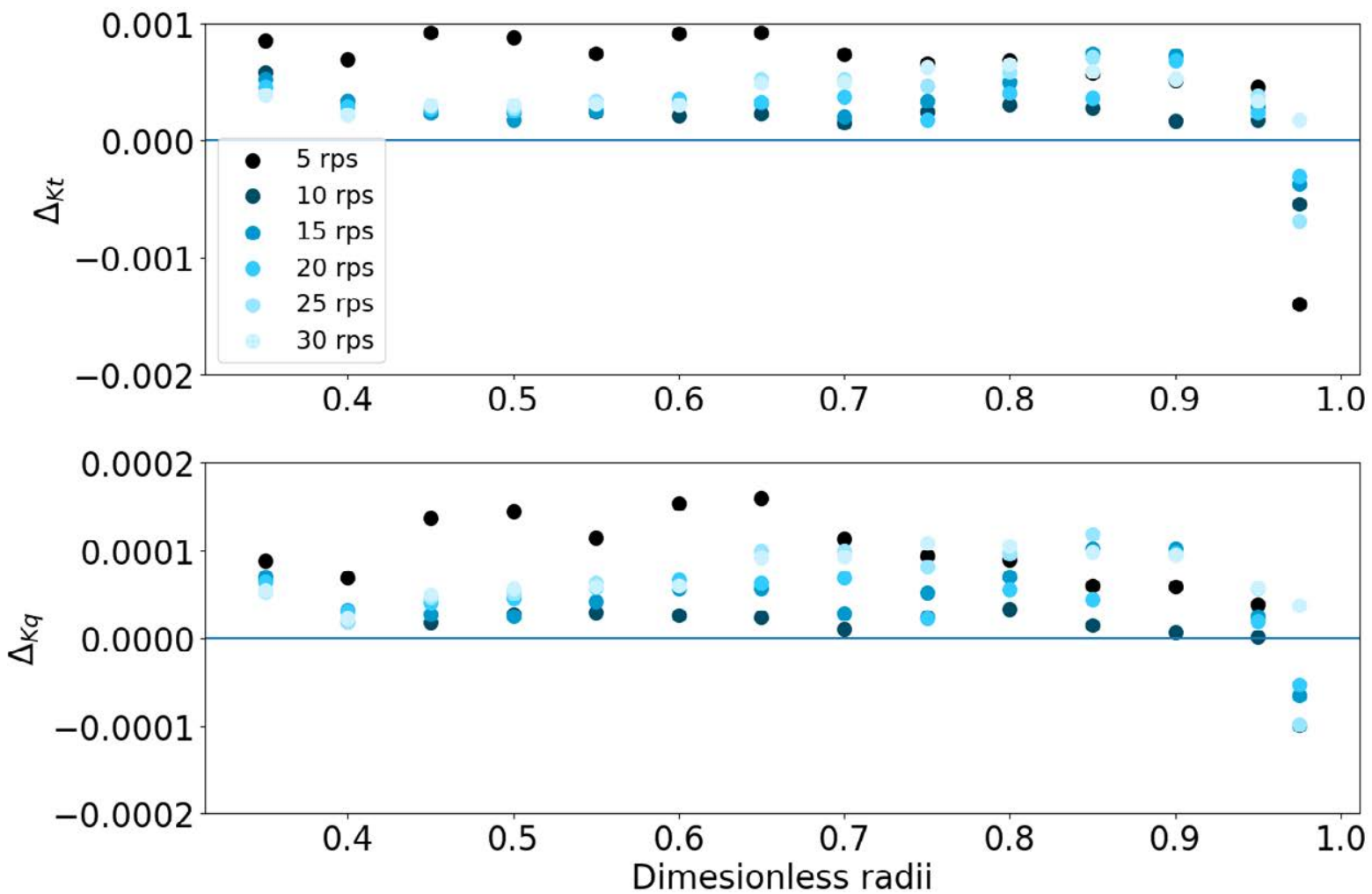

Figure 3.34: CONV02 propeller $\Delta K_{t}$ and $\Delta K_{q}$ due to the lift scale effects, along the radii for the different rps studied.

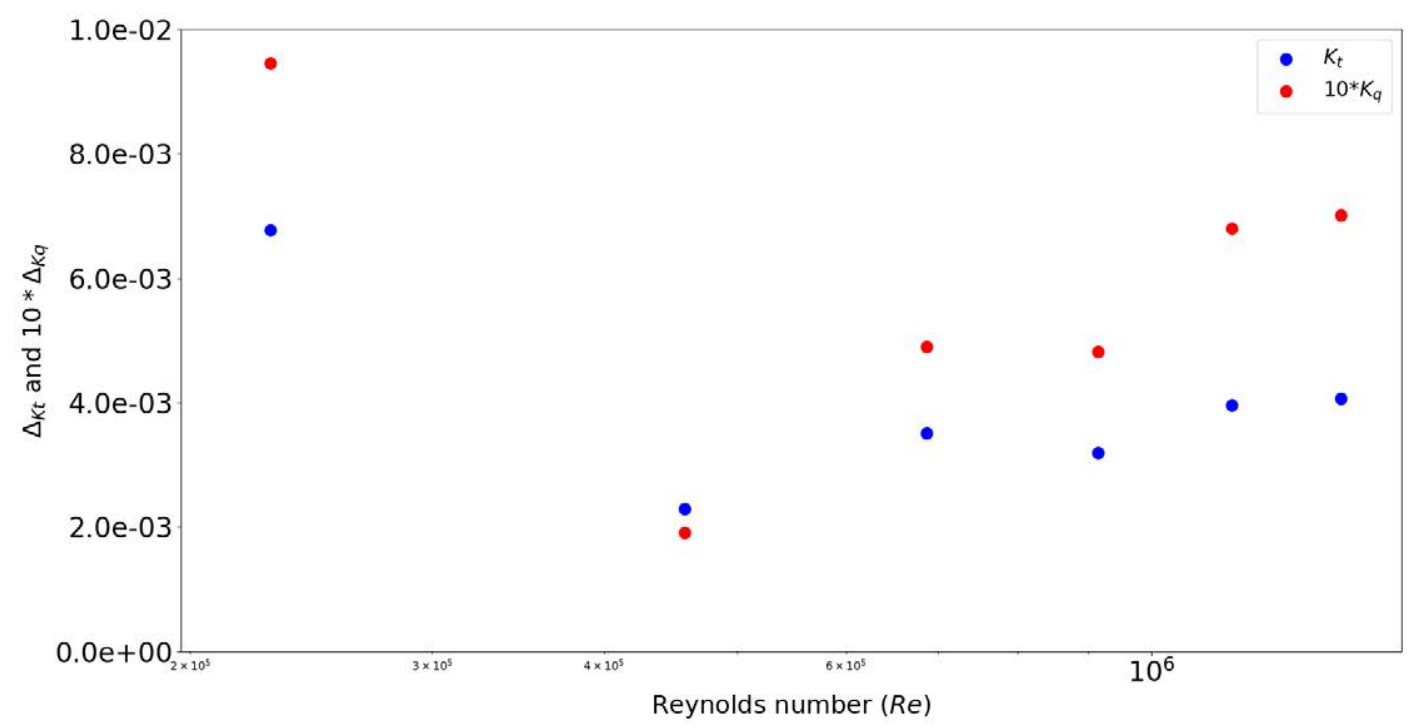

Figure 3.35: CONV02 propeller $\Delta K_{t}$ and $\Delta K_{q}$ due to the lift scale effects, along the radii for the different rps studied. 
as can be appreciated in Figure 3.35, where the integration of the $\Delta K_{t, i}$ and $\Delta K_{q, i}$ along the radii is presented. It is possible to appreciate a minimum in the lift scale effects corrections around $R e=4.8 \cdot 10^{5}$, not only in the magnitude of the values, but also in the difference between $\Delta K_{t}$ and $\Delta K_{q}$, being this crucial for a higher variation in the efficiency values.

\subsection{Analysis of different extrapolation methods.}

In this Subsection, different extrapolation methods for the CONV02 propeller are presented. The main objective is to evaluate the improvement of the models presented in Subsection 3.3 and analyse the effects of introducing the lift scale effects evaluated in Subsection 3.4. A comparison of the extrapolation methods for different $R e$ is also introduced.

\subsubsection{Evaluation of the improvement with the new form factor $k_{M L P}$ and lift scale effects in $K_{t}, K_{q}$ and $\eta_{0}$ coefficients}

In this part, a comparison of the effects introduced by the new form factor for the determination of the drag coefficient (3.7), and the effects introduced by the lift scale effects corrections in the $K_{t}$ and $K_{q}$ coefficients are both presented. The drag coefficient has been obtained from CFD simulations in order to avoid additional modelling errors in the $\Delta C_{f}$ estimation.

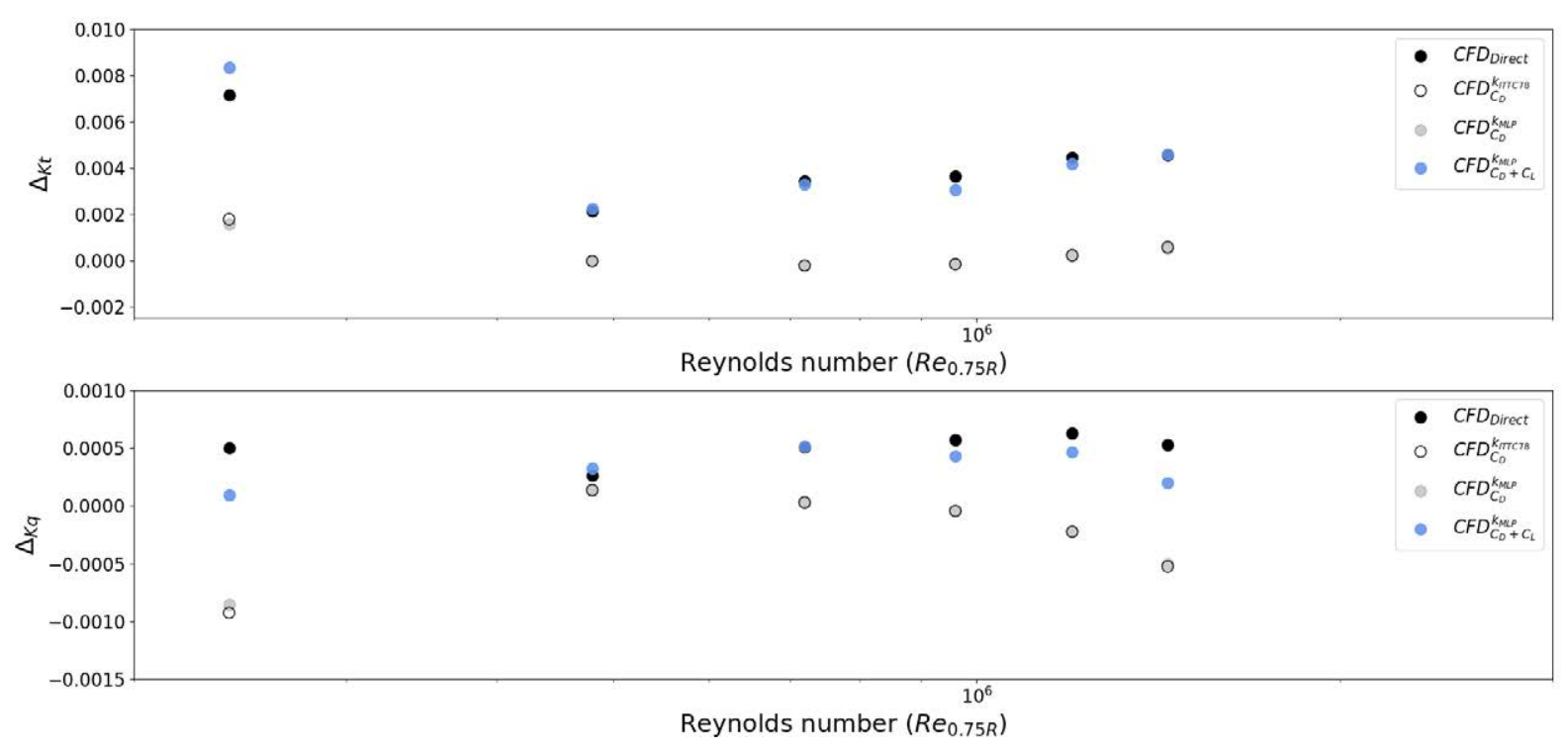

Figure 3.36: $\Delta K_{t}$ and $\Delta K_{q}$ comparison for the extrapolated values using the $k_{I T T C 78}$, the $k_{M L P}$, the $k_{M L P}$ plus lift corrections and the direct $C F D$ simulations for CONV02 propeller. 
Figure 3.36 represents the $\Delta K_{t}$ and $\Delta K_{q}$ for each OWT simulation performed. It is possible to appreciate how the introduction of the lift corrections improve the results quantitatively and qualitatively. The results accurately approximate the direct CFD results when lift corrections are applied. An interesting aspect, in the case where only drag scale effects are applied, is that the minimum differences when compared to the direct CFD results are found at the same Reynolds number $\left(R e \approx 4.7 \cdot 10^{5}\right)$ where the minimum lift corrections appears, see Figure 3.35. It seems that the new form factor used to estimate the drag coefficient of the sections does not introduce large variations in the final correction of $\Delta K_{t}$ and $\Delta K_{q}$. This can be interpreted as that the difference between model and full scale prevails over the exact value for each scale.

The efficiency results for all the methods are presented in Figure 3.37. These results are shown as the ratio between the ones obtained with corrections or directly at full scale simulations and the model scale results, following the Equation 3.10. It is important to highlight, that even when the most approximate results with lift corrections (in terms of $\Delta K_{t}$ and $\Delta K_{q}$ ) it does not necessary entail a better prediction of the $\eta_{0}$ value, considering the better extrapolated points the ones closest to the direct CFD simulations.

$$
\eta_{0}^{\text {ratio }}=\frac{\eta_{0}^{F S}}{\eta_{0}^{M S}}
$$

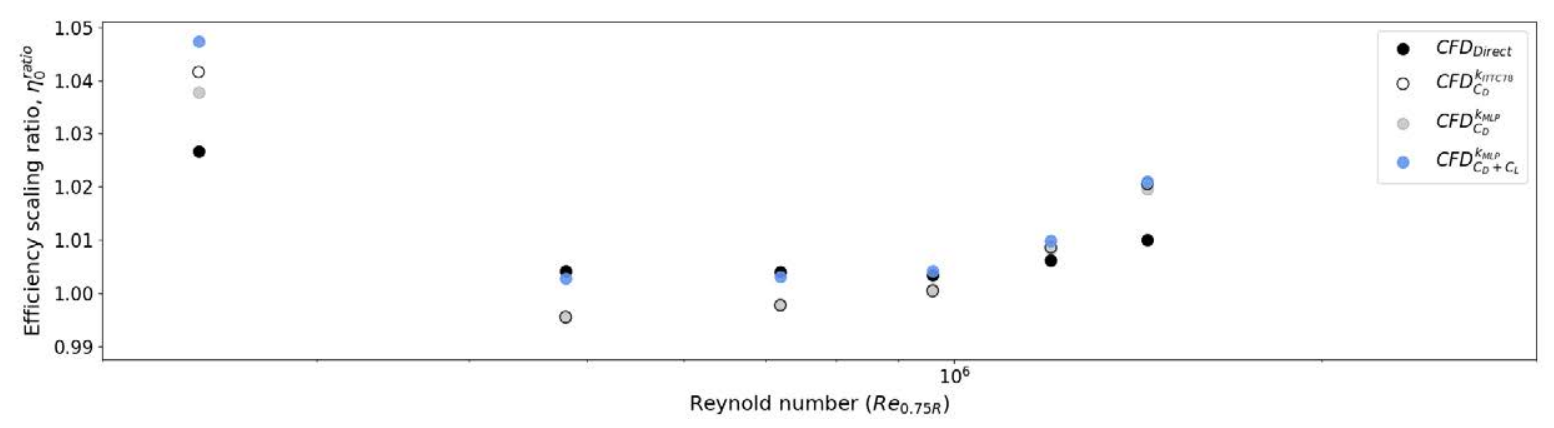

Figure 3.37: CONV02 propeller $\eta_{0}$ comparison for the extrapolated values using the $k_{I T T C 78}$, the $k_{M L P}$, the $k_{M L P}$ plus lift corrections and direct $C F D$ simulations. 


\subsubsection{Comparison of different extrapolation methods with and without lift scale effects.}

In previous Subsections, the improvements introduced by a new form factor estimation and due to the lift corrections were presented for extrapolation based on $\Delta C_{f}$ obtained with CFD results. In this Subsection, a comparison between different extrapolation methods, with and without lift corrections, is presented and compared in turn with results obtained directly from full and model scale simulations. The extrapolation method that obtains the $\Delta C_{f}$ from CFD results is also considered as a reference, being the best approximation that can be obtained supposing that the $C_{f}$ estimations methods match those obtained with CFD.

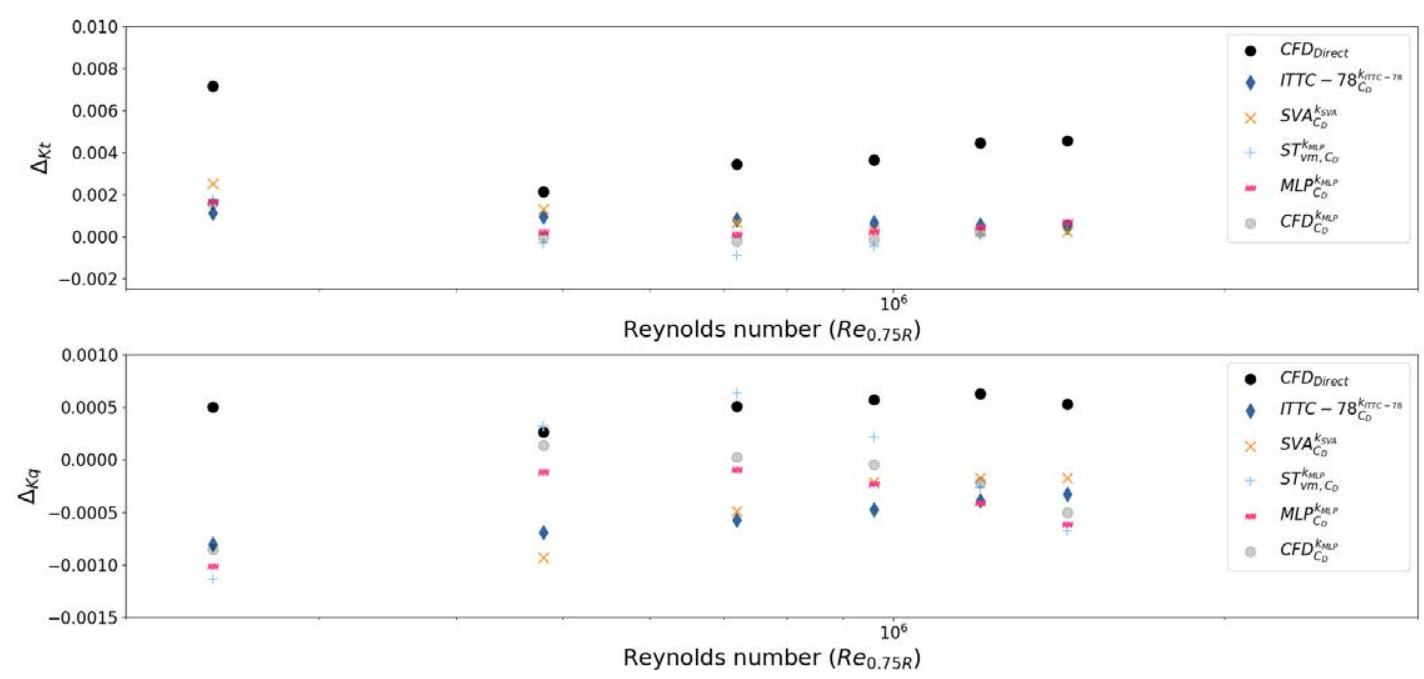

Figure 3.38: CONV02 propeller $\Delta K_{t}$ and $\Delta K_{q}$ comparison for different extrapolation methods without applying lift corrections, and direct $C F D$ simulations.

Figure 3.38 represents the $\Delta K_{t}$ and $\Delta K_{q}$ comparison without the lift scale effects corrections for the ITTC -78 , the $S V A$ and the two new methods presented in Subsection 3.3, STRIPSIS $S_{\text {modified }}$ and $M L P$. It is possible to appreciate in Figure 3.38 that all the methods, in general, under-predict the thrust and torque coefficients and none of them is capable to derive the inflection point present in the $C F D$ results.

Figure 3.39 represents the same as Figure 3.38 but including lift scale effects corrections to $\Delta K_{t}$ and $\Delta K_{q}{ }^{5}$. From Figure 3.39, it can be derived that only the application of lift scale effects corrections lead to curves with an inflection point. In terms of $\Delta K_{t}$, all the methods with the lift scale effects corrections present a very good behaviour. Conversely, in the torque coefficient the ITTC -78 and the $S V A$

\footnotetext{
${ }^{5}$ Lift scale effects are those obtained in Subsection 3.4 with CFD for this particular propeller.
} 


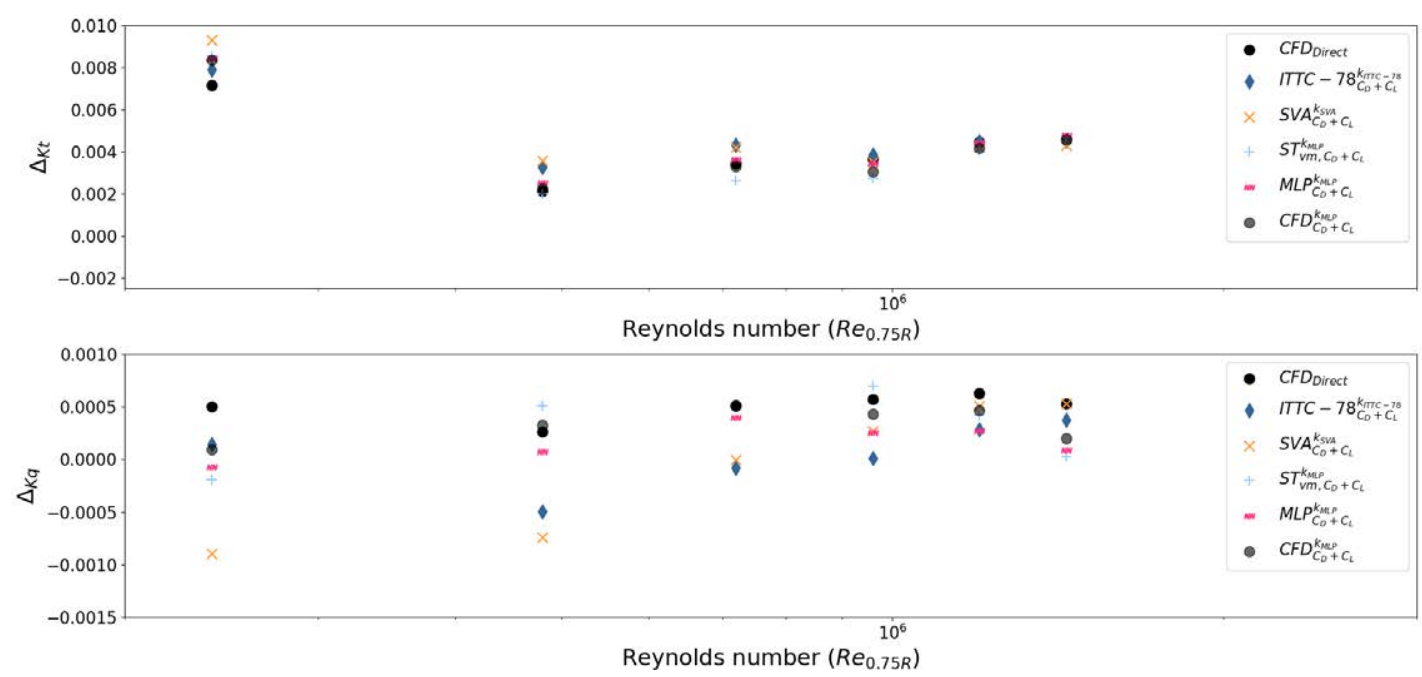

Figure 3.39: CONV02 propeller $\Delta K_{t}$ and $\Delta K_{q}$ comparison for different extrapolation methods applying lift corrections calculated in section 3.4 , and direct $C F D$ simulations.

methods are far from the values obtained with CFD in some of the points. Also, it is important to note that the values of $\Delta K_{q}$ obtained when lift scale effects corrections are not applied are always negative, while in the CFD results the majority are positive. This is a remarkable difference, because it is very difficult, not to say impossible, to have positive values of $\Delta K_{q}$ due to the definition of the friction lines in the $I T T C-78$ and in the $S V A$ formulas.

In order to quantify the improvements of the lift scale effects corrections, direct CFD results are considered as the most realistic and the differences with respect to this model have been calculated in terms of percentage $\Delta K_{t}^{\text {diff }}(\%)$ and $\Delta K_{q}^{\text {diff }}(\%)$, see Equations 3.11 and 3.12 .

$$
\begin{aligned}
\Delta K_{t}^{\text {diff }}(\%) & =100\left(\frac{\Delta K_{t}^{F S}}{\Delta K_{t}^{M S}}-1\right) \\
\Delta K_{q}^{\text {diff }}(\%) & =100\left(\frac{\Delta K_{q}^{F S}}{\Delta K_{q}^{M S}}-1\right)
\end{aligned}
$$

Figures 3.40 and 3.41 represent the values obtained from Equations 3.11 and 3.12 for all the range of Reynolds numbers studied of the propeller CONV02. It is possible to appreciate the huge differences between the values of $\Delta K_{t}^{\text {diff }}$ and $\Delta K_{q}^{\text {diff }}$ when the lift scale effects corrections are applied. The most accurate model, as expected, is the $C F D^{k_{M L P}}$, where the drag coefficient of the sections has been calculated directly with CFD simulations. With respect to the $I T T C-78^{k_{I T T C 78}}$ and $S V A^{k_{S V A}}$ the mean values greatly improve with the lift scale effects corrections but the mean and standard deviation values are still higher than in the rest of the methods ${ }^{6}$.

\footnotetext{
${ }^{6}$ Note that ITTC-78 and SVA methods are based o the equivalent profile theory, and the others are strip-based methods
} 


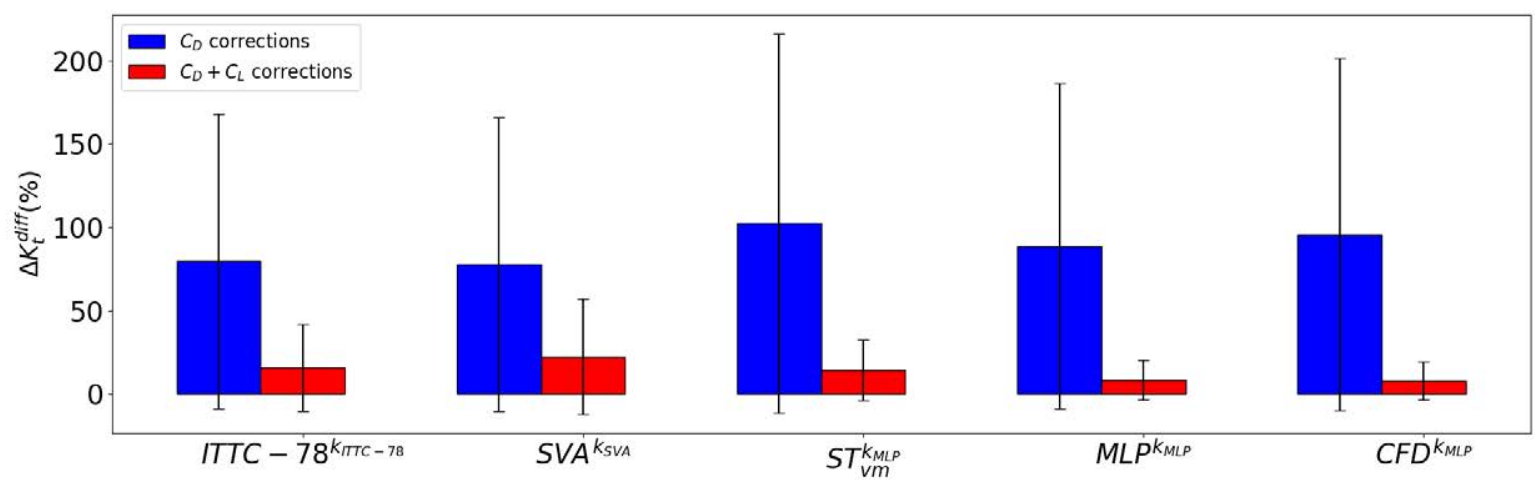

Figure 3.40: Bar diagram representing the results of the Equation 3.12 with and without lift scale effects corrections.

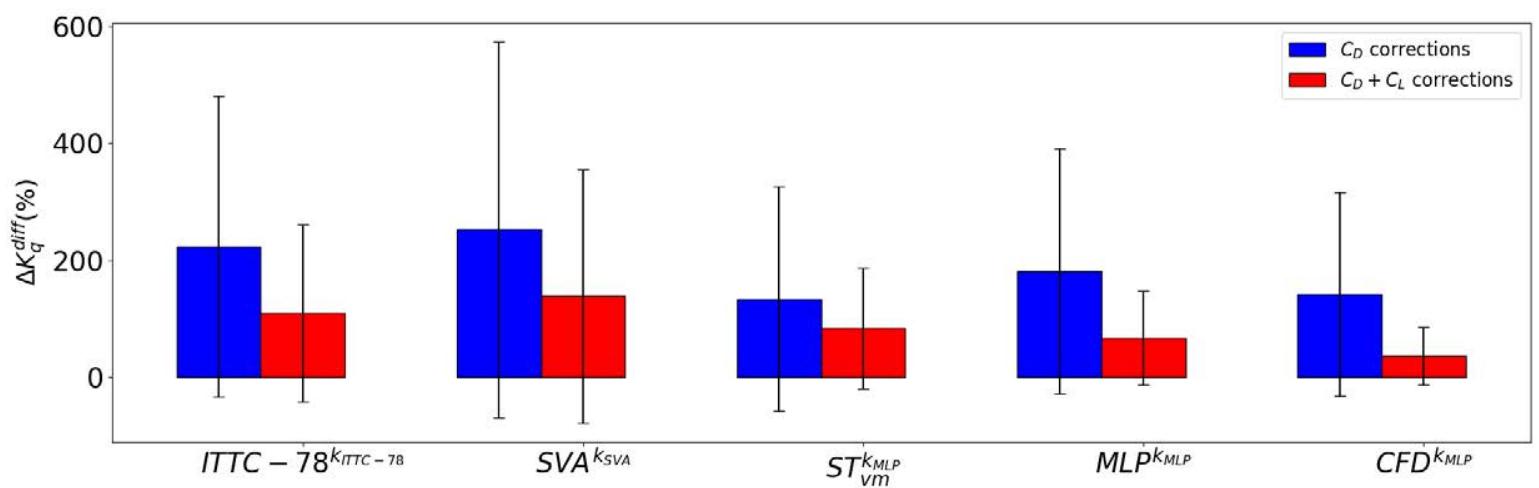

Figure 3.41: Bar diagram representing the results of the Equation 3.11 with and without lift scale effects corrections. 


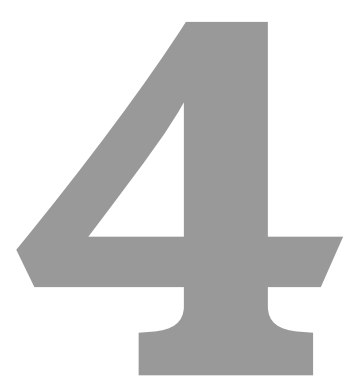

\section{Conclusions}

\section{Contents}

4.1 Summary and concluding remarks $\ldots \ldots \ldots \ldots \ldots \ldots \ldots \ldots \ldots \ldots$

4.2 Future work. . . . . . . . . . . . . . . . . . . . . . 62 
CHAPTER 4. CONCLUSIONS 


\subsection{Summary and concluding remarks}

This thesis has focused on the scale effects that occur in marine propellers when they are simulated at model scale. To carry out this task, it has been necessary the application of transition models, at model scale simulations, to capture the laminar region. Also, the author has considered important the inclusion of the cross-flow transition mechanism in the transition model.

In Subsection 3.1, a comparison of the benefits of the inclusion of the cross-flow transition mechanism is presented. The main conclusion of this section is that, when the cross-flow transition mechanism is added, results of the transition onset location are more accurate than its absence.

In Subsection 3.2, a comparison of different turbulence models for marine propellers simulation is described. The main conclusion for this study is that it is crucial to include transition models in the simulations at model scale propellers when a certain accuracy is searched. Also, the inclusion of transition model is fundamental to appreciate the correct scale effects.

In Subsection 3.3, a variation of the Reynolds number on different propellers has been done. Once this is done, propellers have been radially sectioned and the friction forces, projected on the nose-tail direction for each section. The projected friction forces have been integrated along the chord to find out relations between the Reynolds number and the transition location. Two different methods to estimate the frictional forces have been created and introduced in Appendix D. The most important conclusion of this part is that the transition onset location does not only depend on the Reynolds number demonstrating a high dependency on the geometrical parameters of the blade and the position of the propeller section. On this regard, the tip and blade root sections should be studied in more detail due to the particularities of the flow at these sections.

In Subsection 3.4, the angle of incidence of the flow has been calculated along the radii for the CONV02 propeller. With this angle and the forces acting over the blade, $\Delta K_{t}$ and $\Delta K_{q}$ corrections due to lift scale effects have been calculated for this particular propeller. After this study, the conclusion that lift scale effects do not play a negligible role in the OWT corrections can be derived. Additionally, a certain Reynolds number range where the lift scale effects are minimum has been found, being around $R e_{0.7 R} \approx 5 \cdot 10^{5}$. For higher Reynolds number the lift scale effects would decrease again.

In Subsection 3.5, first an analysis of the improvement that the new form factor $\left(k_{M L P}\right)$ and the lift scale effects introduce to the friction coefficient difference between full and model scale (directly obtained with CFD) is presented. Second a comparison of the different extrapolation methods, with and 
without the inclusion of lift scale effects corrections, for the CONV02 propeller is presented. The main conclusions from the first point are:

- Without the lift scale effects corrections it is not possible to match, starting from model scale simulations, the results directly obtained with full scale CFD simulations.

- The new form factor to estimate the drag coefficient does not improve the results in terms of $\Delta K_{t}$ and $\Delta K_{q}$ coefficients, even though it drastically improves the prediction of the drag coefficient.

With respect to the second point of Subsection 3.5, the main conclusion is, once more, that there is not an extrapolation method capable to be close enough to the full scale CFD results without the inclusion of the lift scale effects corrections, at least for this particular propeller. Additionally it was found that scale effects in $\Delta K_{q}$ are positive, while in the majority of extrapolation methods are negative by definition. This can be justified by the fact that corrections are based on $\Delta C_{f}$ between model and full scale and friction lines are always decreasing with the Reynolds number.

\subsection{Future work.}

Analysing the results obtained in this thesis, there is future work that should be done, which may be summarized as follows:

- Perform a study with different levels of roughness and turbulence intensity to find out the effects on the friction coefficients and consequently in the difference between model and full scale.

- Perform a meta-study with different kind of propellers to find out lift scale effects corrections, being flow and geometrical sensitive.

- Improve the MLP presented in Subsection 3.3, by enlarging the database of the propellers used to train the neural network. With the method presented in Subsection 3.4, it will be possible to create a new MLP that estimates directly the $C_{D}$ and leave up the need to use form factors.

- Investigate in more detail the effects in the tip and blade root sections of the propeller. In this thesis, the minimum and maximum radii to train the MLP has been 0.35 and $0.975 R$ respectively. A more detailed analysis should be done to apply this method to 'Tip-Raked' and CLT propellers due to the different flow at the tip regions. Also the propellers with large hub-diameter ratio such as the $C P P$ propellers should be study in more detail.

- All the simulations in this thesis until Section 3.3 have been done for the design advance coefficient, so the conclusions are relative to the operating point of the propellers. It may be carried out an investigation to evaluate the influence of off-design conditions. 
- Perform Measurements of the Turbulent Intensity of the water in a normal Open Water Test and in the cavitation tunnel.

Part of this future work will try to be the answer to the next questions:

- What happens if roughness and a certain level of turbulent intensity is included in the simulations? Does it change the conclusions referent to the drag and lift scale effects?

- How much turbulence intensity level there is in an towing tank? and in a cavitation tunnel?.

- Is possible to create an extrapolation model based on Artificial Neural Network capable to include drag and lift scale effects for any kind of propeller? 


\section{Bibliography}

Abu-Ghannam, B. and Shaw, R. (1980). Natural transition of boundary layers - the effects of turbulence, pressure gradient, and flow history. Journal of Mechanical Engineering Science, 22:213-228.

Baltazar, J., Rijpkema, D., and Falcao de Campos, J. (2018). On the use of the $\gamma-r e_{\theta}$ transition model for the prediction of the propeller performance at model-scale. Ocean Engineering, 170:6 - 19, ISSN: 0029-8018, DOI: https://doi.org/10.1016/j.oceaneng.2018.10.005, http://www. sciencedirect.com/science/article/pii/S0029801818307133.

Bhattacharyya, A., Krasilnikov, V., and Steen, S. (2016). Scale effects on open water characteristics of a controllable pitch propeller working within different duct designs. Ocean Engineering, 112:226 - 242, ISSN: 0029-8018, DOI: http://dx.doi.org/10.1016/j.oceaneng.2015.12.024, //www.sciencedirect.com/science/article/pii/S0029801815006782.

Bhattacharyya, A., Neitzel, J., Steen, S., Abdel-Maksoud, M., and Krasilnikov, V. (2015). Influence of flow transition on open and ducted propeller characteristics. In Fourth International Symposium on Marine Propulsors, SMP.

Bugalski, T., Streckwall, H., and Szantyr, J. (2013). Critical review of propeller performance scaling methods, based on model experiments and numerical calculations. In POLISH MARITIME RESEARCH, volume 20, pp. 71-79.

Bulten, N. and Stoltenkamp, P. (2017). Full scale cfd: the end of froude-reynolds battle. In Fith International Symposium on Marine Propulsors, SMP-17.

Dagenhart, J. (1992). Crossflow stability and transition experiments in a swept-wing flow. PhD thesis, Faculty of the Virginia Polytechnic Institute and State University.

Di Pasquale, D., Rona, A., and Garret, S. (2009). A selective review of CFD transition models. AAIA.

Funeno, I. (2002). On viscous flow around marine propellers. Journal of the Kansai Society of Naval Architects, 238. 
Gaggero, S., Gonzalez-Adalid, J., and Sobrino, M. P. (2016). Design and analysis of a new generation of $\{C L T\}$ propellers. Applied Ocean Research, 59:424 - 450, ISSN: 0141-1187, DOI: http://dx.doi.org/10.1016/j.apor.2016.06.014, http://www. sciencedirect. com/science/article/pii/s0141118716302279.

Gonzalez-Adalid, J., Perez-Sobrino, M., Gaggero, S., and Gennaro, G. Moran-Guerrero, A. (2018). The use of modern computational tools in the design process of unconventional propellers for performance prediction and full-scale extrapolation. In Proceedings of NAV 2018: 19th International Conference on Ship and Maritime Research.

Gonzalez-Adalid, J., Perez-Sobrino, M., Moran-Guerrero, A., Gonzalez-Gutierrez, L., Riola Rodríguez, J., Diaz-Hernandez, J., Quereda-Laviña, R., and Soriano-Gomez, C. (2016). New generation of clt ${ }^{\circ}$ propellers. In Maritime Technology and Engineering 3, MARTECH.

Goodfellow, I., Bengio, Y., and Courville, A. (2016). Deep Learning. MIT Press. http://www. deeplearningbook. org.

Helma, S. (2015). An extrapolation method suitable for scaling of propellers of any design. In Fourth International Symposium on Marine Propulsors, SMP-15.

Helma, S., Streckwall, H., and Richter, J. (2017). The effect of propeller scaling methodology on the performance prediction. In Fith International Symposium on Marine Propulsors, SMP-17.

ITTC (2011). 1978 ITTC Performance prediction method 7.5-02-03-01.4.

Klose, R., Schulze, R., and Hellwig-Rieck, K. (2017). Investigation of prediction methods for tip rake propellers. In Fith International Symposium on Marine Propulsors, SMP-17.

Kuiper, G. (1981). Cavitation inception on ship propeller models. PhD thesis, Ph.D. thesis, Delft University of Technology.

Kuiper, G. (2000). Improving full scale ship powering performance predictions by application of propeller leading edge roughness, part 1: effect of leading edge roughness on propeller performance. PhD thesis, Master thesis, Delft University of Technology.

Langtry, R. (2006). A Correlation-Based Transition Model using Local Variables for Unstructured Parallelized CFD codes. $\mathrm{PhD}$ thesis, $\mathrm{PhD}$ thesis, University of Stuttgart.

Langtry, R. and Menter, F. (2009). Correlation-Based Transition modelling for Unstructured Parallelized Computational Fluid Dynamics Codes. AIAA, 47:2894-2906.

Langtry, R., Sengupta, K., Yeh, D., and Dorgan, A. (2015). Extending the $\Gamma-R e_{\Theta}$ Local Correlation based Transition Model for Crossflow Effects. AIAA, 47. 
Lerbs, H. W. (1951). On the effects of scale and roughness on free running propellers. JASNE, (63).

Malan, P., Suluksna, K., and Juntasaro, E. (2009). Calibrating the $\Gamma-R e_{\Theta}$ transition model for commercial CFD. AIAA.

Menter, F., Langtry, R., Likki, S., Suzen, Y., Huang, P., and Volker, S. (2004). A Correlation-based Transition Model Using Local Variables Part 1- Model Formulation. ASME.

Menter, F., Langtry, R., and Volker, S. (2006). Transition modelling for general purpose CFD codes. Flow, Turbulence and Combustion, 77:277-303.

Meyne, K. (1968). Experimentelle und theorische betrachtungen zum masstabseffekt bei modellpropeller-untersuchungen. Schiffstechnik, (15).

Moran-Guerrero, A., Gonzalez-Adalid, J., Gonzalez-Gutierrez, L., and Perez-Sobrino, M. (2017). Open water results comparison for three propellers with transition model, applying crossflow effect, and its comparison with experimental results. In Fith International Symposium on Marine Propulsors, SMP17.

Moran-Guerrero, A., Gonzalez-Gutierrez, L. M., Oliva-Remola, A., and Diaz-Ojeda, H. R. (2018). On the influence of transition modeling and crossflow effects on open water propeller simulations. Ocean Engineering, 156:101 - 119, ISSN: 0029-8018, DOI: https://doi.org/10.1016/j.oceaneng.2018.02.068, http://www.sciencedirect.com/science/ article/pii/S0029801818302245.

Moran-Guerrero, A., J., and Gonzalez-Gutierrez (2016). Nnatac, preliminary results. In Seventh annual Airbus DiPaRT 2016.

Muller, C. and Herbst, F. (2014). Modelling of crossflow-induced transition based on local variables. In 11th World Congress on Computational Mechanics, WCCM XI.

Perez-Sobrino, M., Gonzalez-Adalid, J., and Quereda-Laviña, R. (2005). Scale effects in model tests with clt propellers. In 27th Motorship Marine Propulsion Conference.

Perez-Sobrino, M., Gonzalez-Adalid, J., Quereda-Laviña, R., Soriano-Gomez, C., Gennaro, G., and Moran-Guerrero, A. (2016). A new performance prediction procedure for propellers with unconventional tip shape. In 12th International Conference on Hydrodynamics, 18 - 23 September 2016,.

Ponkratov, D. and Constanttinos, Z. (2015). Validation of ship scale cfd self-propulsion simulation by the direct comparison with sea trials results. In Fourth International Symposium on Marine Propulsors, SMP-15. 
Sánchez-Caja, A., González-Adalid, J., Pérez-Sobrino, M., and Sipilä, T. (2014). Scale effects on tip loaded propeller performance using a ranse solver. Ocean Enginnering, (88):607-617.

Saric, W., Reed, H. L. ., and White, E. B. (2003). Stability and transition of three-dimensional boundary layers. Annual Review Fluid Meshanics, pp. 413-440.

Streckwall, H., Greitsch, L., Müller, J., Scharf, M., and Bugalski, T. (2013). Development of a strip method proposed as new standard for propeller performance scaling. Ship Technology Research, 50:2:58-69, http://dx.doi.org/10.1179/str.2013.60.2.002.

SVA (2011). Open water tests with the model propeller vp1304. Technical report, http://www . sva-potsdam.de/wp-content/uploads/2016/04/SVA_report_3752.pdf.

SVA (2016). Ittc propeller benchmark, tip raked propeller - p1727. Technical report, https://www . sva-potsdam.de/wp-content/uploads/2017/09/Report-4487-ITTC-Benchmark-TipRake.pdf.

Vukcević, V., Jasak, H., Gatin, I., and Uroić, T. (2017). Ship scale self propulsion cfd simulation results compared to sea trial measurements. In VII International Conference on Computational Methods in Marine Engineering, MARINE 2017.

Wilcox, D. C. (2006). An introduction to statistical modeling of extreme values. Springer series in Statistics. Springer, 3 edition, ISBN: 1928729088, https://www.amazon.com/ Turbulence-Modeling-Third-David-Wilcox/dp/1928729088. 


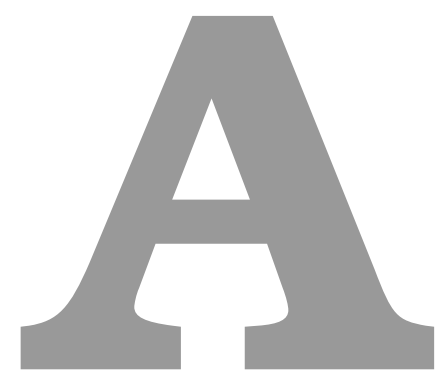

Geometries 
APPENDIX A. GEOMETRIES 


\section{A.1 Propellers studied}

For this thesis different propellers have been used. In Section 3.2 the VP1304, P1727 and CLT02 were used and in Section 3.3, the propellers were the VP1304, P1727, CLT01, CLT03, CLT04 and CONV04, to complete a good amount of data available to train the MLP. For the purpose of the studies presented in Sections 3.4 and 3.5 the CONV02 propeller was used. The main reason to use this propeller is that, it has not been previously used to train the MLP.

The Controllable Pitch Propeller (CPP) VP1304 and the Tip-Raked Fixed Pitch Propeller (FPP) P1727 were used to study the scale effects for a conventional and unconventional propeller, see SVA (2011), SVA (2016), the main characteristics are presented in Table A.1. The CLT01, CLT02 are CPP propellers that were used as reference and final optimized propeller respectively in the COINCIDENTE project. Both propellers were designed for the BAM class Spanish corvettes.

The CLT03 is a CPP propeller that was designed for a container ship, while CLT04, CONV02 and CONV04 are CPP propellers designed for a POD housing applications. The main characteristics of this propellers can be found in Tables A.2 and A.3.
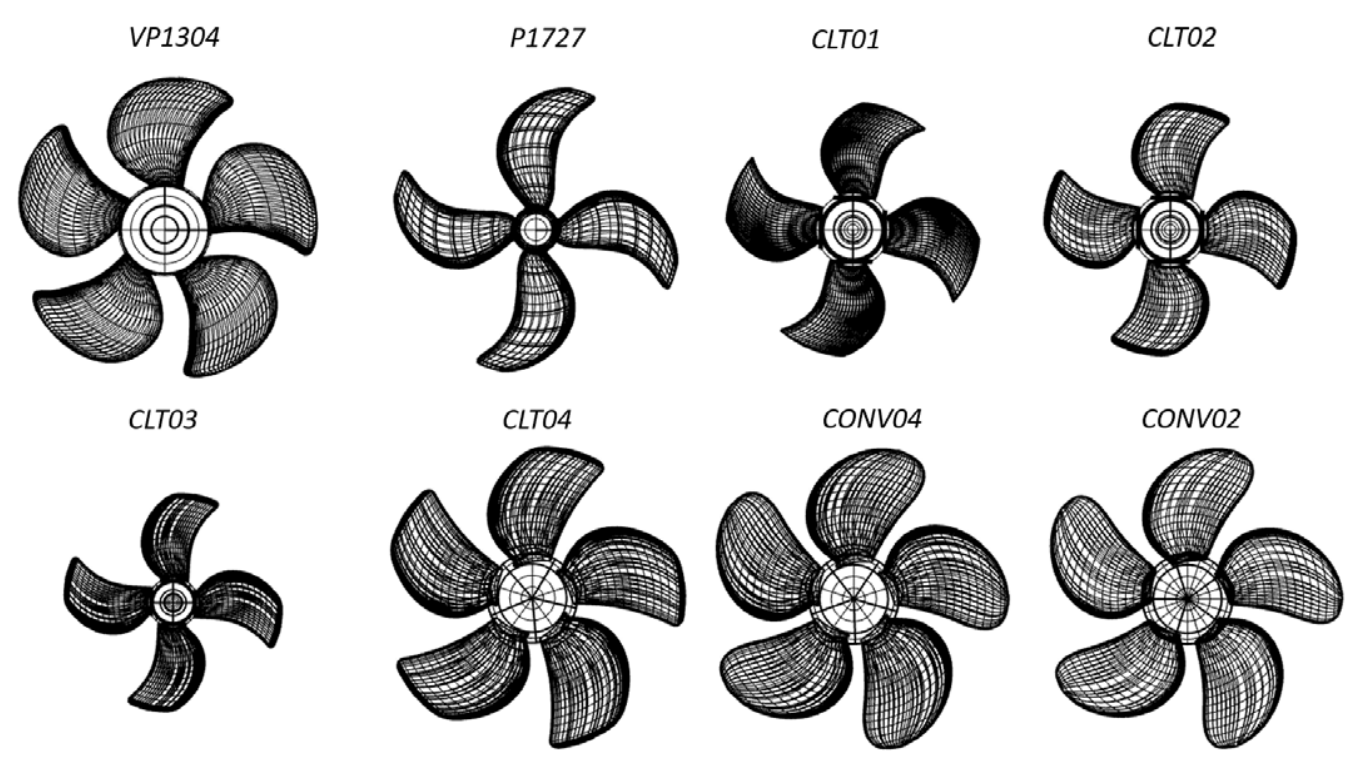

Figure A.1: Frontal view for all the propellers studied. 


\begin{tabular}{|c|c|c|c|}
\hline & & VP1304 & P1727 \\
\hline Type & & CPP & FPP, Tip-Raked \\
\hline Diameter [mm] & $D$ & 250.000 & 238.600 \\
\hline Pitch at $0.75 \mathrm{R}[\mathrm{mm}]$ & $P_{0.75}$ & 407.400 & 191.000 \\
\hline Chord at $0.75 \mathrm{R}[\mathrm{mm}]$ & $C r_{0.75}$ & 106.300 & 55.600 \\
\hline Thickness at $0.75 \mathrm{R}[\mathrm{mm}]$ & $T h_{0.75}$ & 3.800 & 2.900 \\
\hline Area Ratio & $A e / A o$ & 0.779 & 0.444 \\
\hline Blades & $z$ & 5 & 4 \\
\hline Scale ratio & $\lambda$ & 12.000 & 31.428 \\
\hline Reynolds range & $R e_{0.75 R}$ & {$\left[2.99 \cdot 10^{5}, 1.79 \cdot 10^{6}\right]$} & {$\left[1.41 \cdot 10^{5}, 1.41 \cdot 10^{6}\right]$} \\
\hline RPS range & rps & {$[5,30]$} & {$[5,50]$} \\
\hline Full scale results & & YES & YES \\
\hline
\end{tabular}

Table A.1: Main geometric characteristics of the propellers used for the ITTC benchmark test case. 


\begin{tabular}{|c|c|c|c|c|c|}
\hline & & CLT01 & CLT02 & CLT03 & CLT04 \\
\hline Type & & CPP, CLT & CPP, CLT & CPP, CLT & CPP, CLT \\
\hline Diameter [mm] & $D$ & 207.831 & 207.831 & 207.831 & 250.000 \\
\hline Pitch at $0.75 \mathrm{R}[\mathrm{mm}]$ & $P_{0.75}$ & 227.711 & 242.331 & 227.711 & 296.033 \\
\hline Chord at $0.75 \mathrm{R}[\mathrm{mm}]$ & $C r_{0.75}$ & 67.813 & 71.084 & 67.813 & 85.242 \\
\hline Thickness at $0.75 \mathrm{R}[\mathrm{mm}]$ & $T h_{0.75}$ & 3.265 & 2.630 & 3.265 & 2.163 \\
\hline Area Ratio & $A e / A o$ & 0.551 & 0.563 & 0.551 & 0.709 \\
\hline Blades & $z$ & 4 & 4 & 4 & 5 \\
\hline Scale ratio & $\lambda$ & 16.6 & 16.6 & 16.6 & 24.0 \\
\hline Reynolds range & $R e_{0.75 R}$ & {$\left[3.08 \cdot 10^{5}, 9.24 \cdot 10^{5}\right]$} & {$\left[3.23 \cdot 10^{5}\right]$} & {$\left[3.08 \cdot 10^{5}, 9.24 \cdot 10^{5}\right]$} & {$\left[2.33 \cdot 10^{5}, 1.40 \cdot 10^{6}\right]$} \\
\hline RPS range & rps & {$[10,30]$} & [15] & {$[10,50]$} & {$[5,30]$} \\
\hline Full scale results & & YES & NO & YES & YES \\
\hline
\end{tabular}

Table A.2: Main geometric characteristics of CLT propeller used in this thesis. 


\begin{tabular}{|c|c|c|c|}
\hline & & CONV04 & CONV02 \\
\hline Type & & CPP & CPP \\
\hline Diameter [mm] & $D$ & 254.167 & 254.167 \\
\hline Pitch at $0.75 \mathrm{R}[\mathrm{mm}]$ & $P_{0.75}$ & 290.096 & 295.708 \\
\hline Chord at $0.75 \mathrm{R}[\mathrm{mm}]$ & $C r_{0.75}$ & 96.688 & 86.304 \\
\hline Thickness at $0.75 \mathrm{R}[\mathrm{mm}]$ & $T h_{0.75}$ & 1.992 & 2.2958 \\
\hline Area Ratio & $A e / A o$ & 0.736 & 0.665 \\
\hline Blades & $z$ & 5 & 5 \\
\hline Scale ratio & $\lambda$ & 24.0 & 24.0 \\
\hline Reynolds range & $R e_{0.75 R}$ & {$\left[2.69 \cdot 10^{5}, 1.61 e 6\right]$} & {$\left[2.4 \cdot 10^{5}, 1.44 e 6\right]$} \\
\hline RPS range & rps & {$[5,30]$} & {$[5,30]$} \\
\hline Full scale results & & YES & YES \\
\hline
\end{tabular}

Table A.3: Rest of the conventional propellers used in this thesis. 


\section{A.2 M2355 profile}

The M2355 profile was a common profile for the aeronautical industry. This profile has been used to make a comparison between the utilization of transition model with and without the inclusion of crossflow transition mechanism. The main geometrical and hydrodynamics parameters are presented in Table A.4.

\begin{tabular}{|l|c|}
\hline \multicolumn{2}{|c|}{ M2355 Profile } \\
\hline Profile & \\
\hline Chord [m] & 0.435 \\
\hline Span & 2 Chord \\
\hline Reynolds & $5.0 \cdot 10^{6}$ \\
\hline Angle of attack & $0^{\circ}-3^{\circ}$ each $0.5^{\circ}$ \\
\hline Sweep angle & $25^{\circ}$ \\
\hline
\end{tabular}

Table A.4: Main parameters of the M2355 profile. 
Contour plot and sectional data distributions 
In this appendix the sectional data and pressure distribution of certain propellers used for sections 3.3 and 3.5. Particularly, the propellers showed are the VP1304, the P1727 and the CONV04. The rest are not shown due to confidentiality reasons.

\section{B.1 VP1304.}

In Figure B.1 the friction coefficient over the propeller VP1304 is shown. Figures B.2, B.3, B.4, B.5 represent some of the radial sections for $C_{p}$ and $C_{f, n t}$.

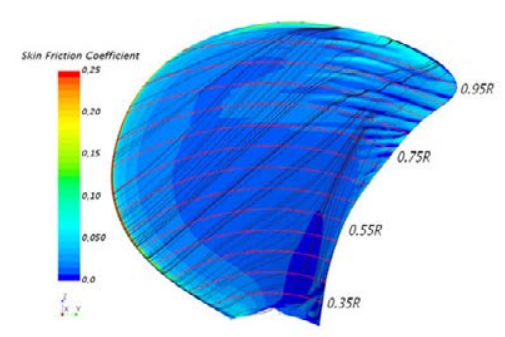

(a) $5 \mathrm{rps}$

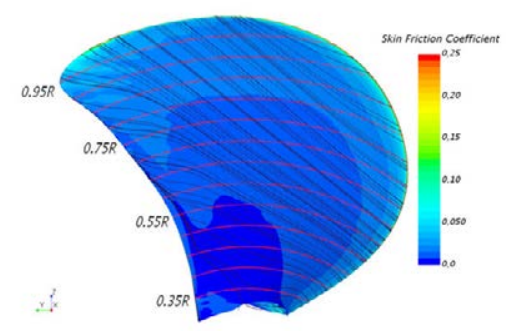

(d) 5 rps

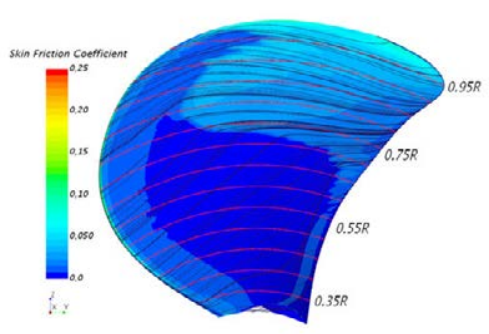

(b) $15 \mathrm{rps}$

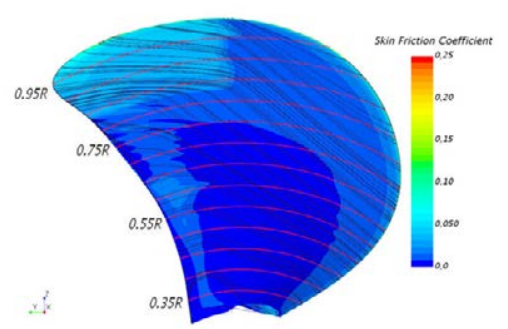

(e) $15 \mathrm{rps}$

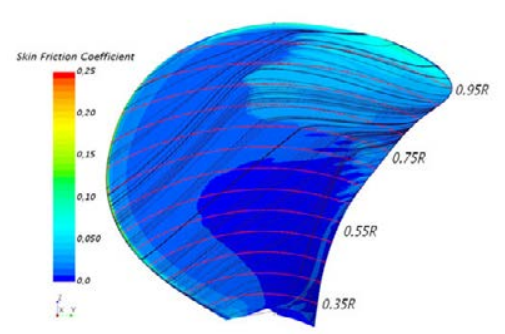

(c) $25 \mathrm{rps}$

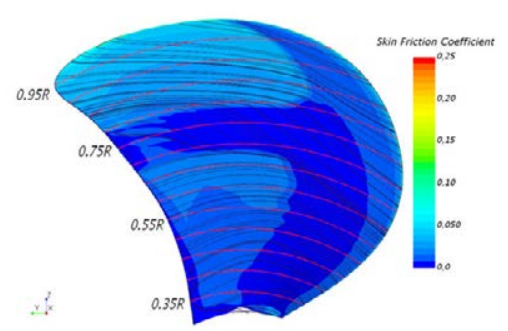

(f) $25 \mathrm{rps}$

Figure B.1: Skin friction contour plot and streamlines representation over the suction (top) and pressure (bottom) sides for the VP1304 propeller at $J=1.0$ for different Reynolds numbers. 

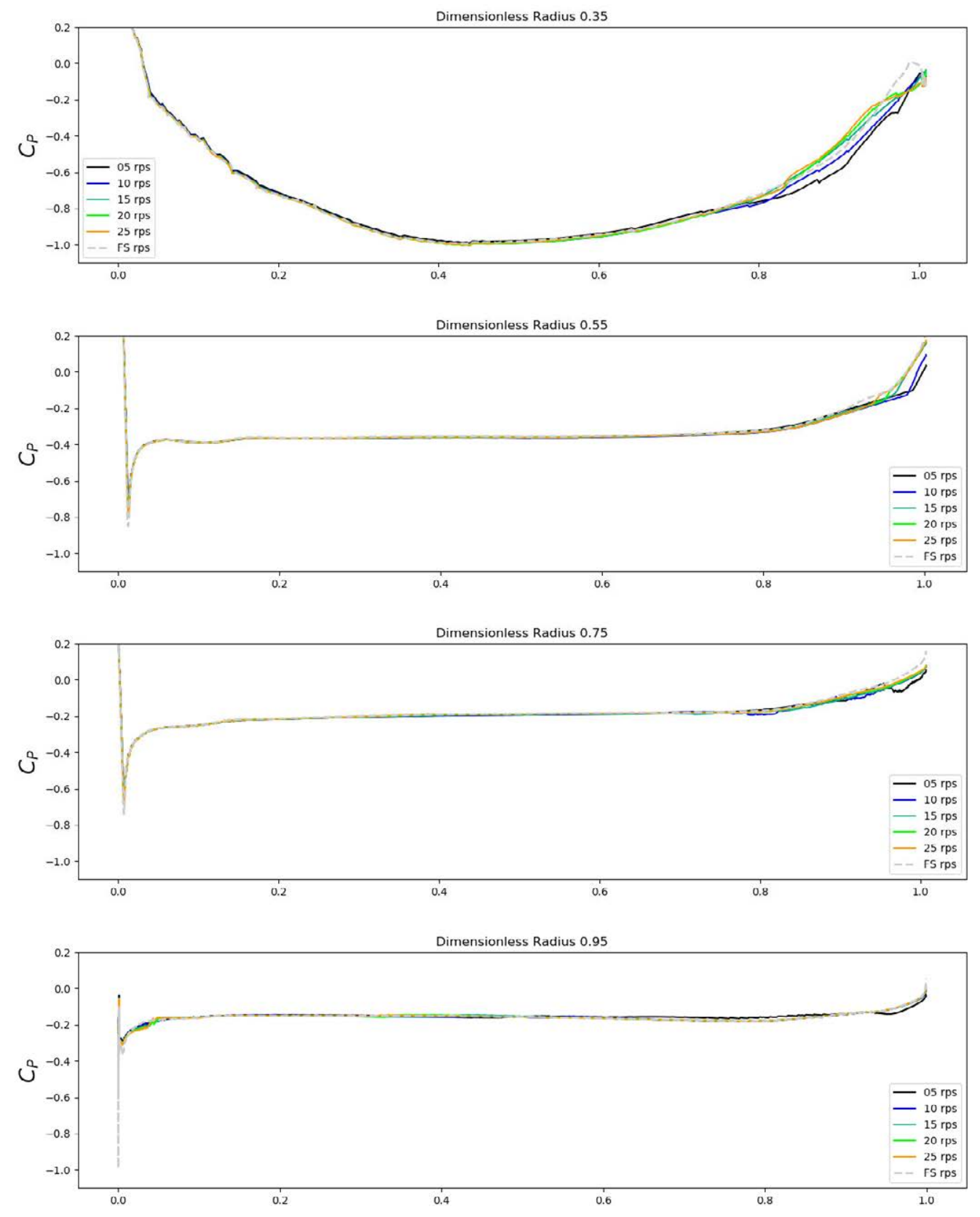

Figure B.2: Suction side of the $C_{p}$, for different radii of the VP1304 propeller. 


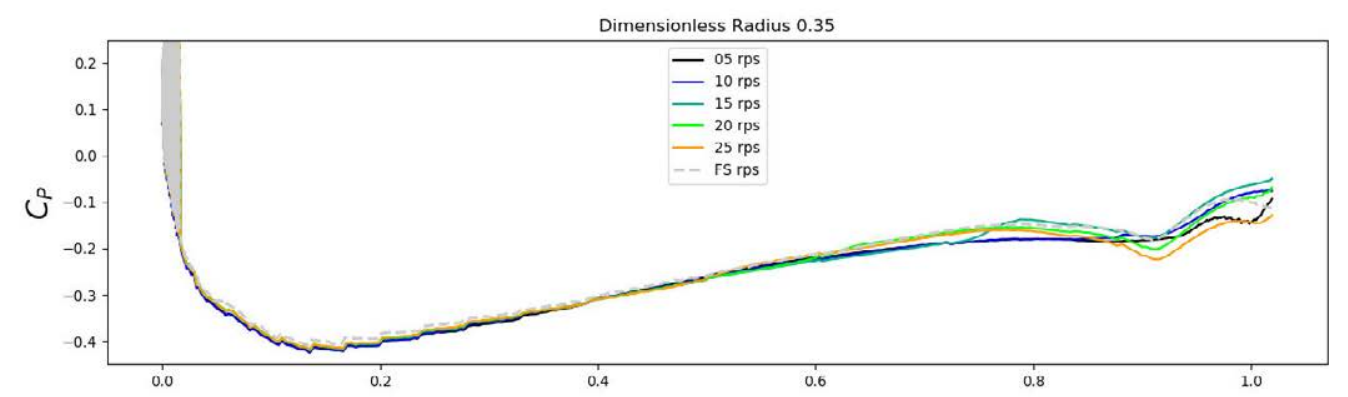

Dimensionless Radius 0.55
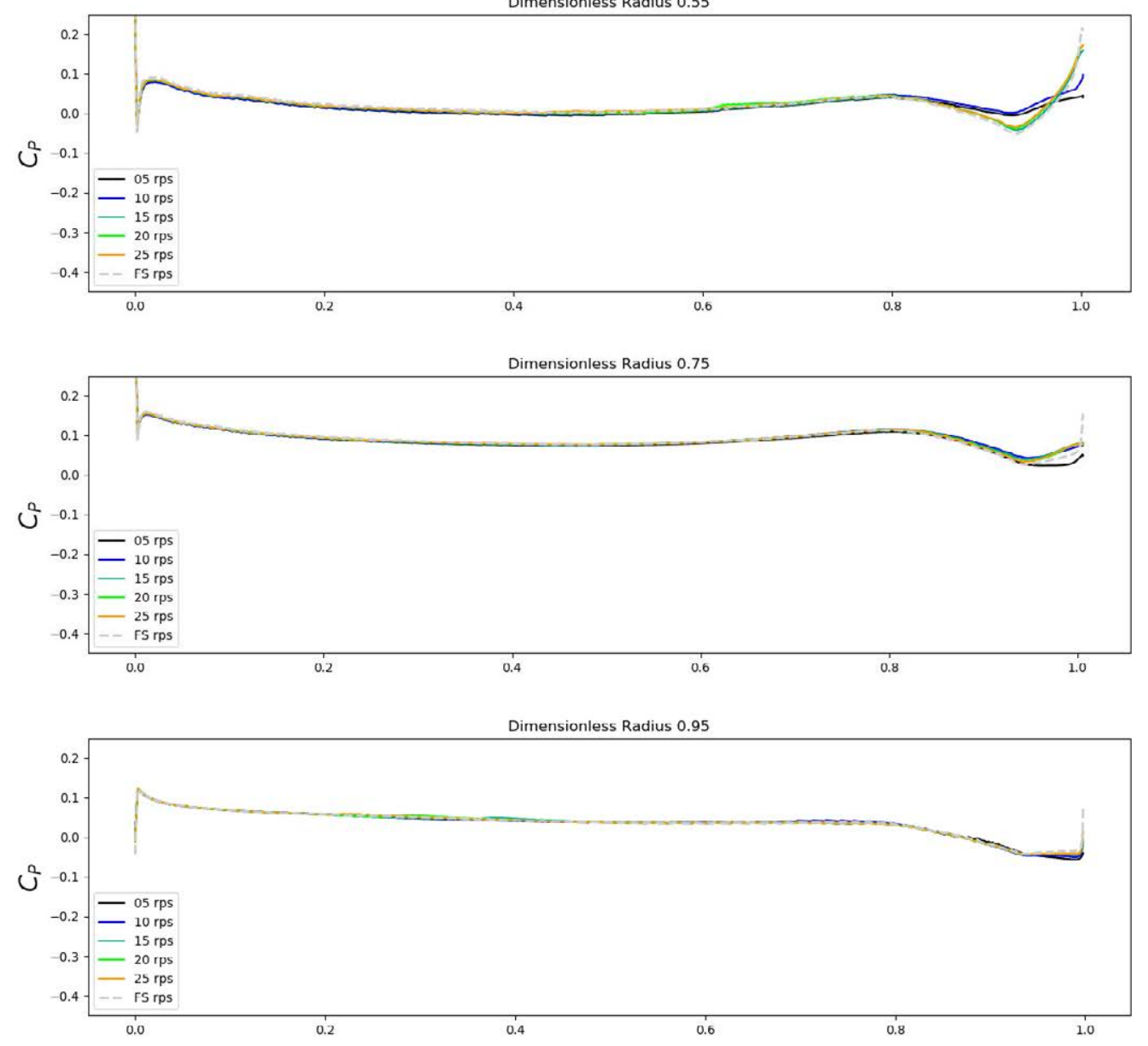

Figure B.3: Pressure side of the $C_{p}$, for different radii of the VP1304 propeller. 

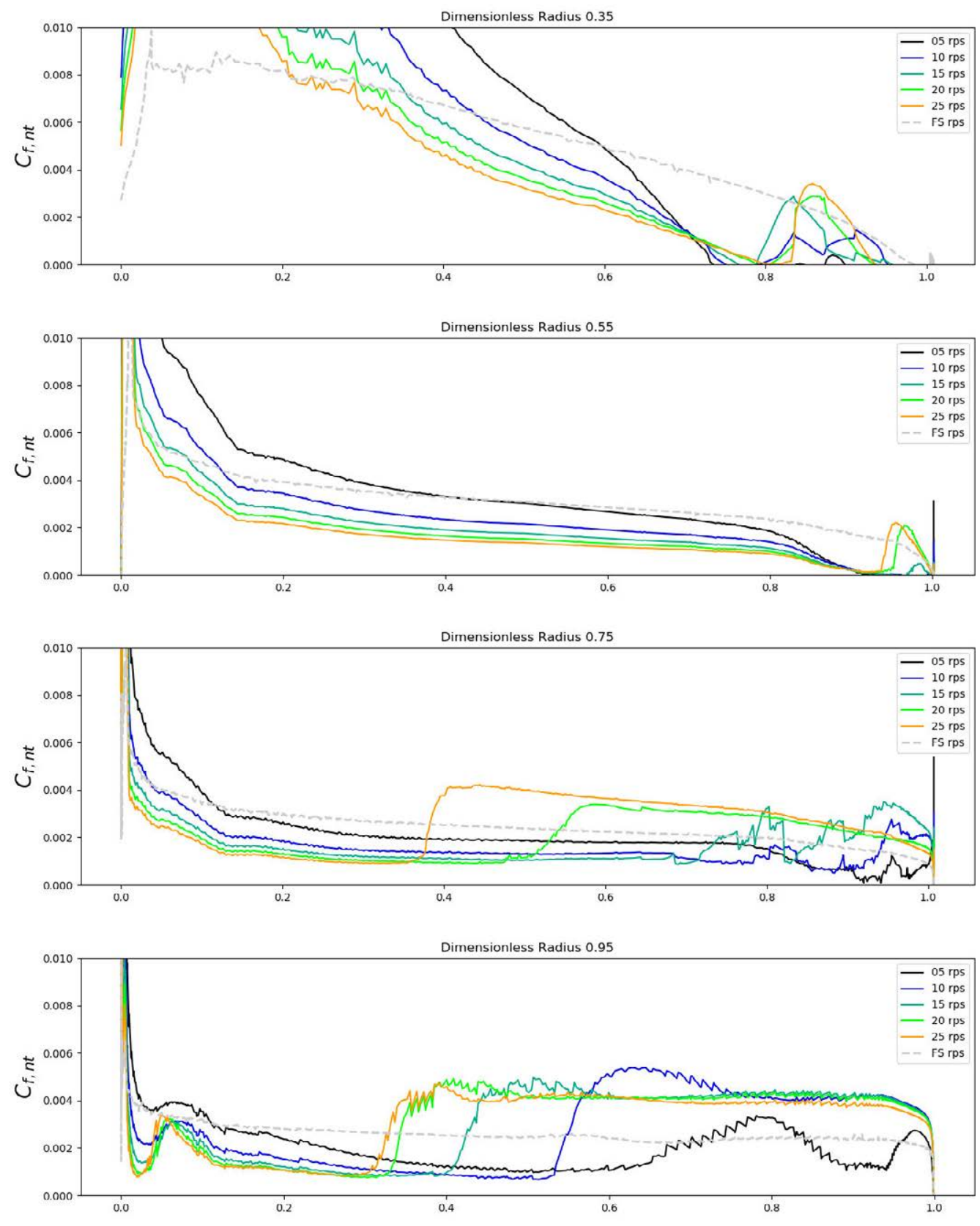

Figure B.4: Suction side of the $C_{f, n t}$, for different radii of the VP1304 propeller. 

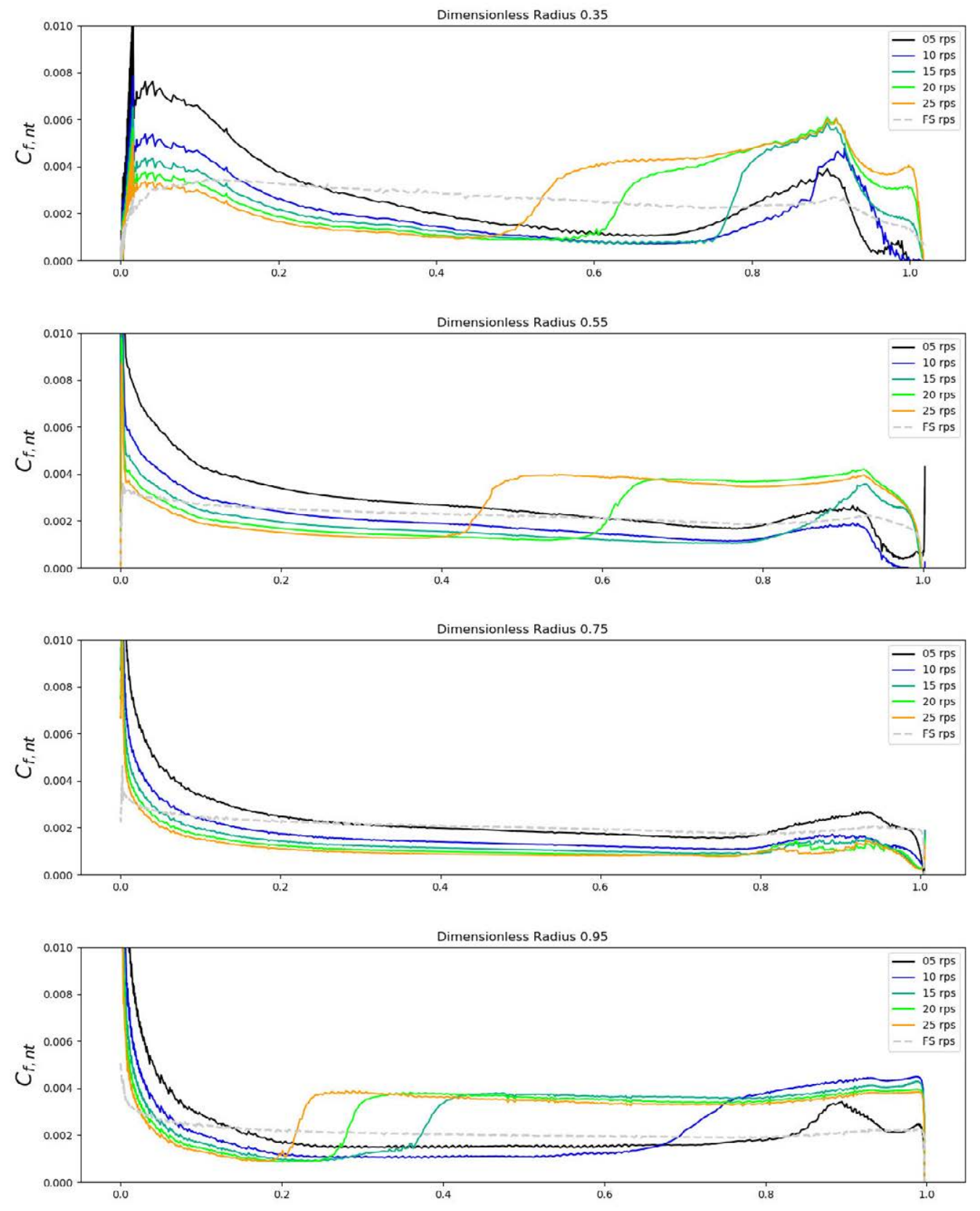

Figure B.5: Pressure side of the $C_{f, n t}$, for different radii of the VP1304 propeller. 


\section{B.2 P1727.}

In Figure B.6 the friction coefficient over the propeller P1727 is shown. Figures B.7, B.8, B.9, B.10 represent some of the radial sections for $C_{p}$ and $C_{f, n t}$.

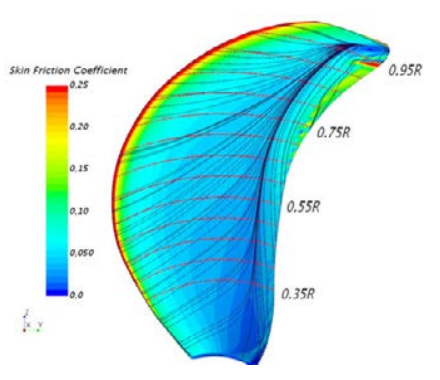

(a) 5 rps

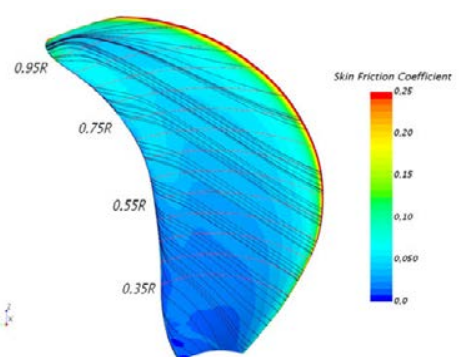

(d) $5 \mathrm{rps}$

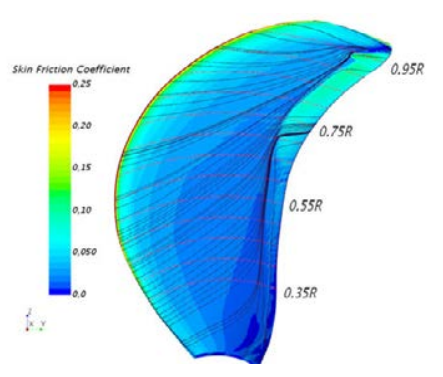

(b) $15 \mathrm{rps}$

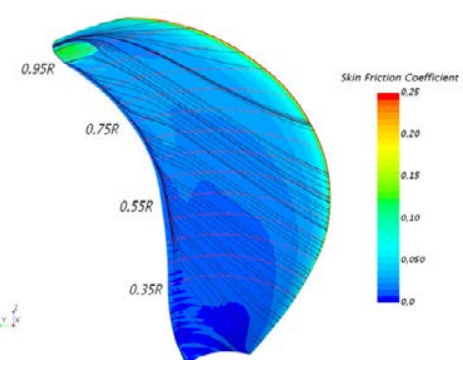

(e) $15 \mathrm{rps}$

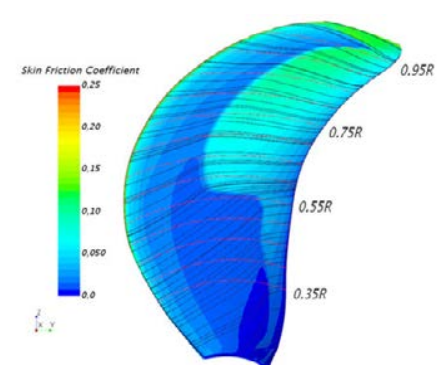

(c) $25 \mathrm{rps}$

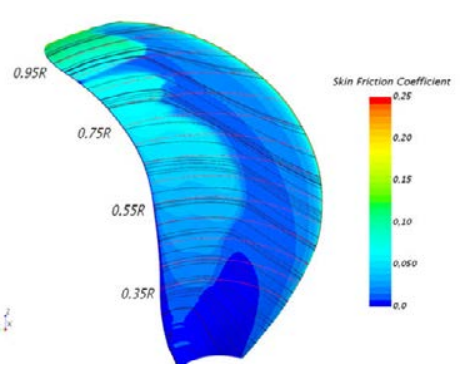

(f) $25 \mathrm{rps}$

Figure B.6: Skin friction contour plot and streamlines representation over the suction (top) and pressure (bottom) sides for the P1727 propeller at $J=0.565$ for different Reynolds numbers. 

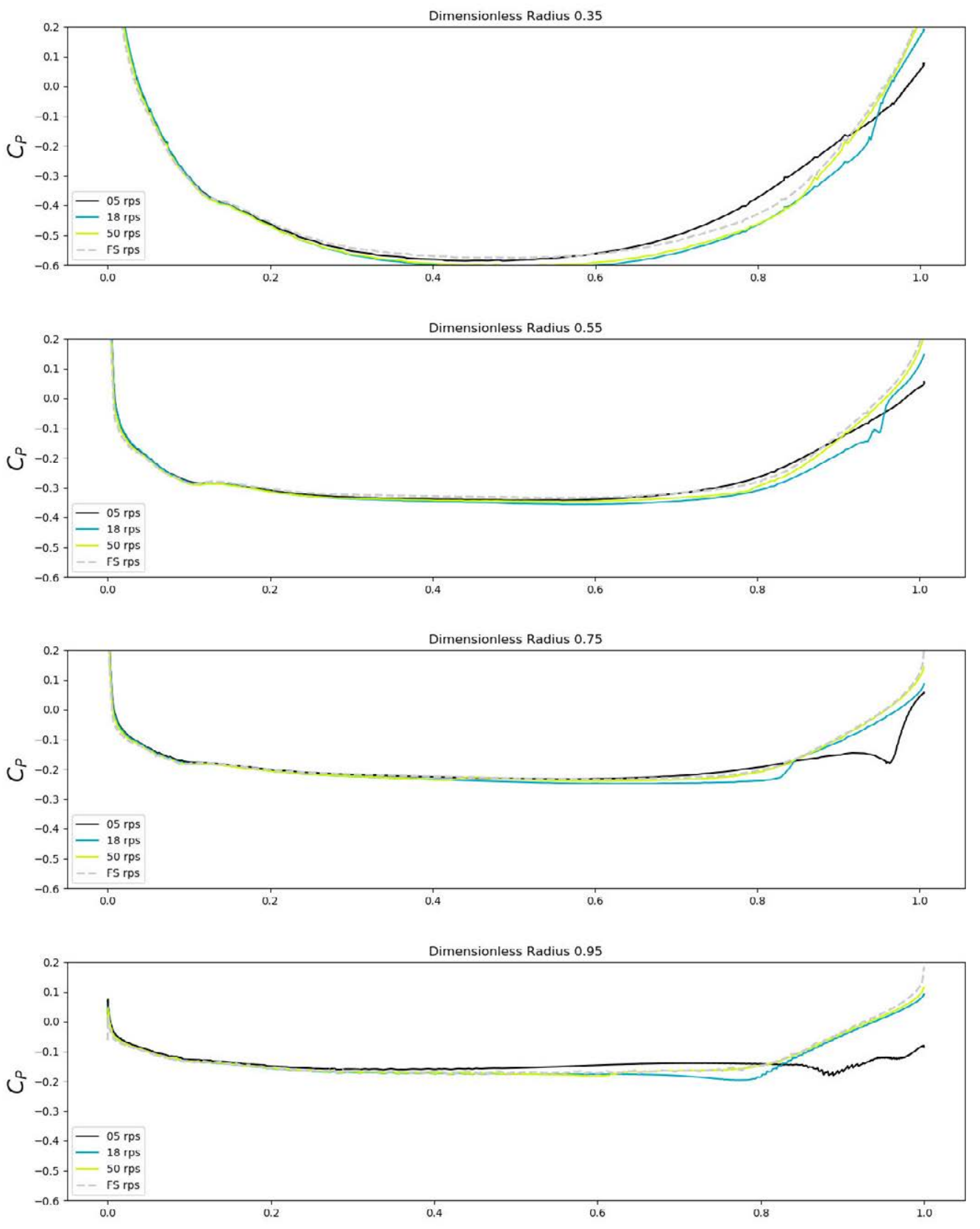

Figure B.7: Suction side of the $C_{p}$, for different radii of the $\mathrm{P} 1727$ propeller. 

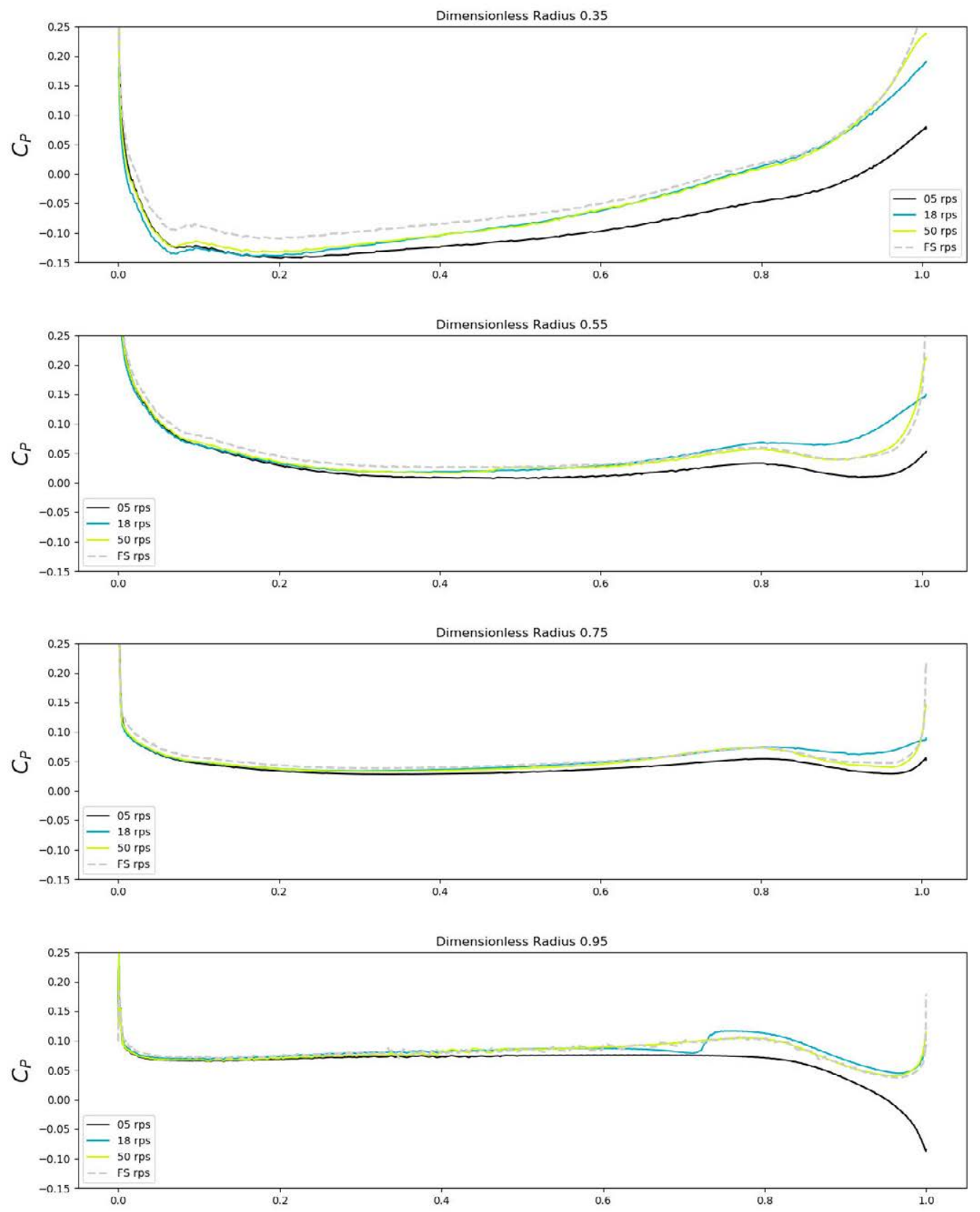

Figure B.8: Pressure side of the $C_{p}$, for different radii of the $\mathrm{P} 1727$ propeller. 

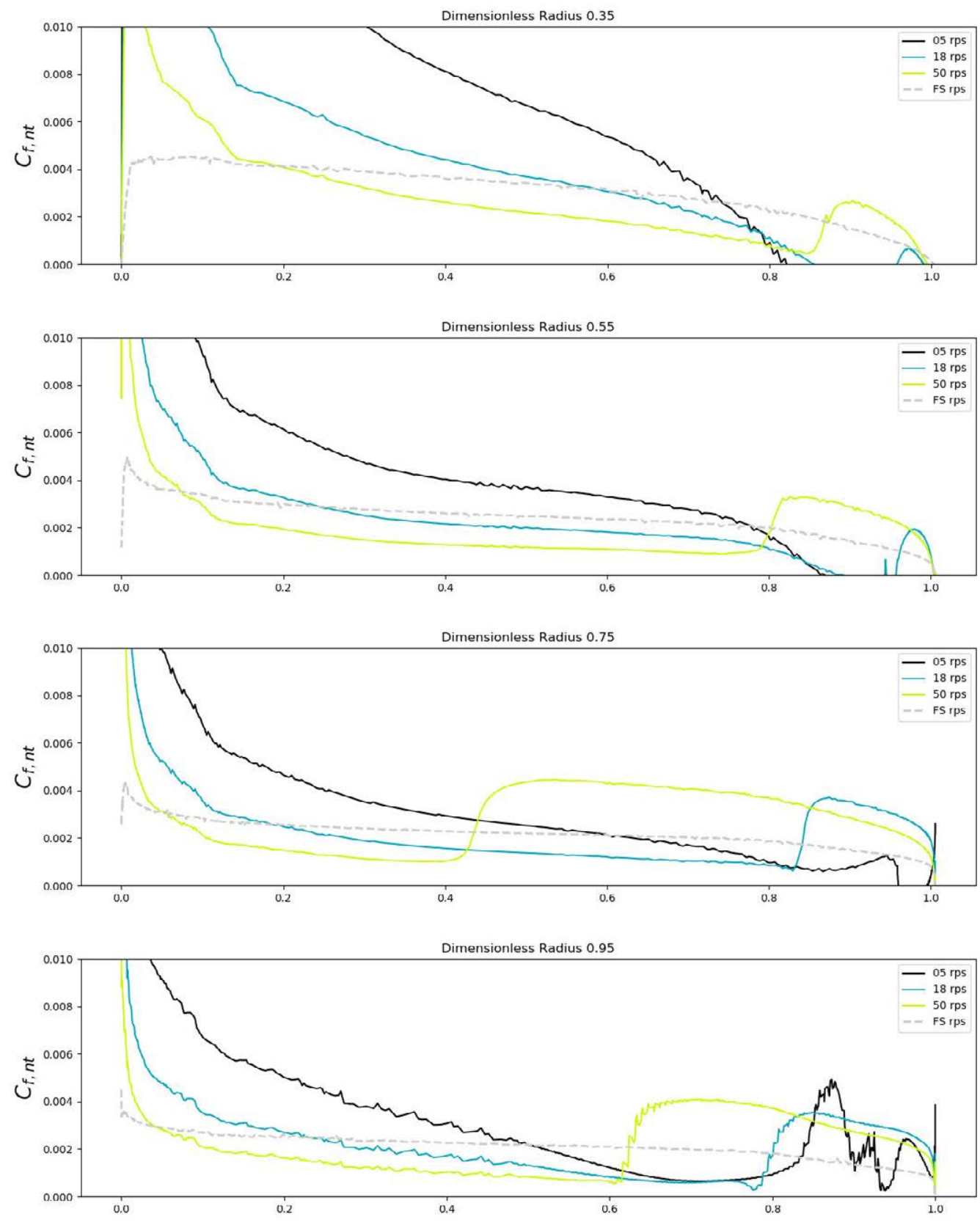

Figure B.9: Suction side of the $C_{f, n t}$, for different radii of the $\mathrm{P} 1727$ propeller. 

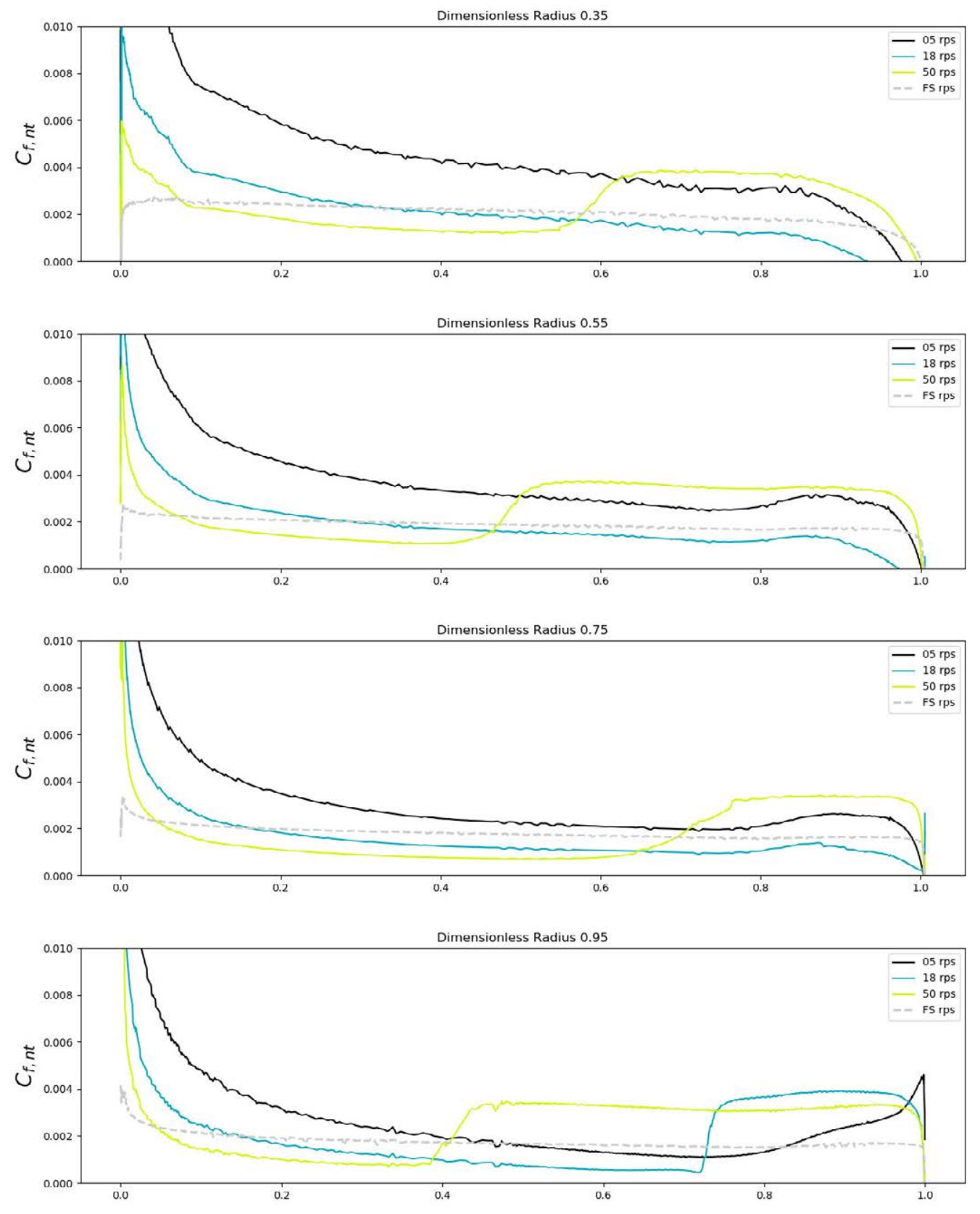

Figure B.10: Pressure side of the $C_{f, n t}$, for different radii of the $\mathrm{P} 1727$ propeller. 


\section{B.3 CONV04.}

In Figure B.11 the friction coefficient over the propeller CONV04 is shown. Figures B.12, B.13, B.14, B. 15 represent some of the radial sections for $C_{p}$ and $C_{f, n t}$.

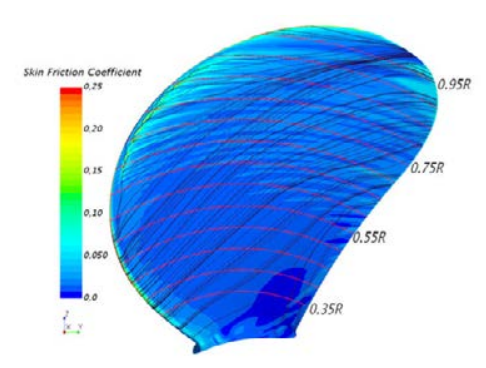

(a) 5 rps

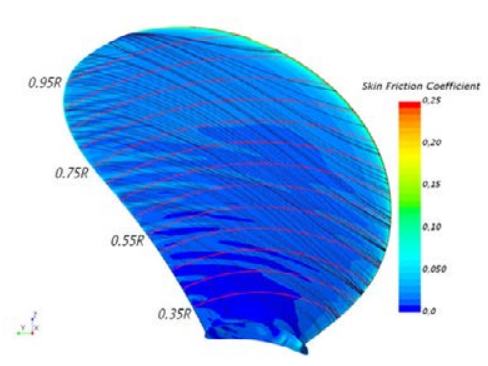

(d) $5 \mathrm{rps}$

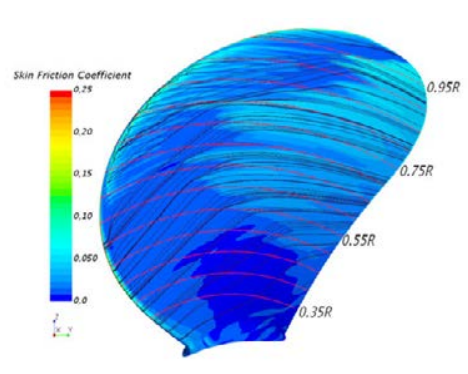

(b) $15 \mathrm{rps}$

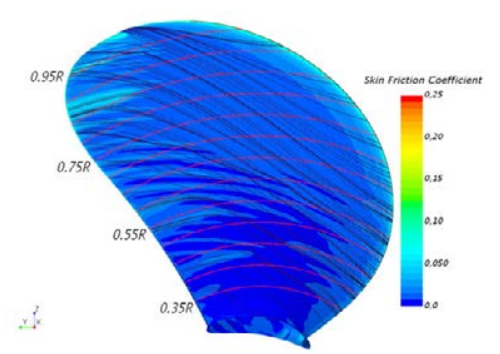

(e) $15 \mathrm{rps}$

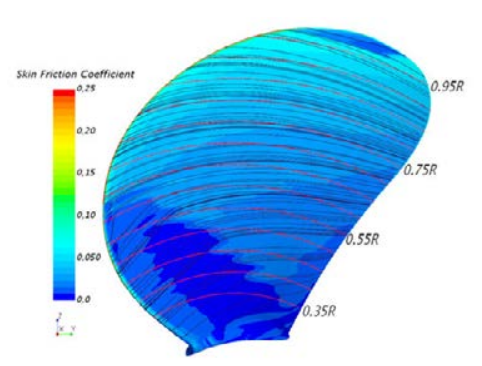

(c) $25 \mathrm{rps}$

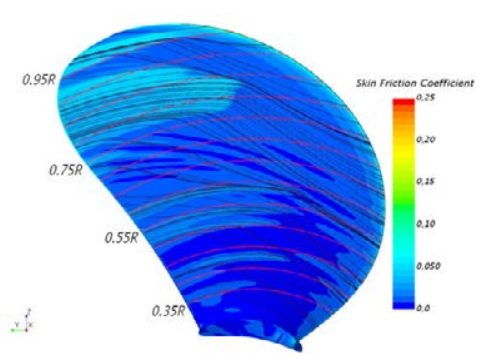

(f) $25 \mathrm{rps}$

Figure B.11: Skin friction contour plot and streamlines representation over the suction (top) and pressure (bottom) sides for the CONV04 propeller at $J=0.8105$ for different Reynolds numbers. 

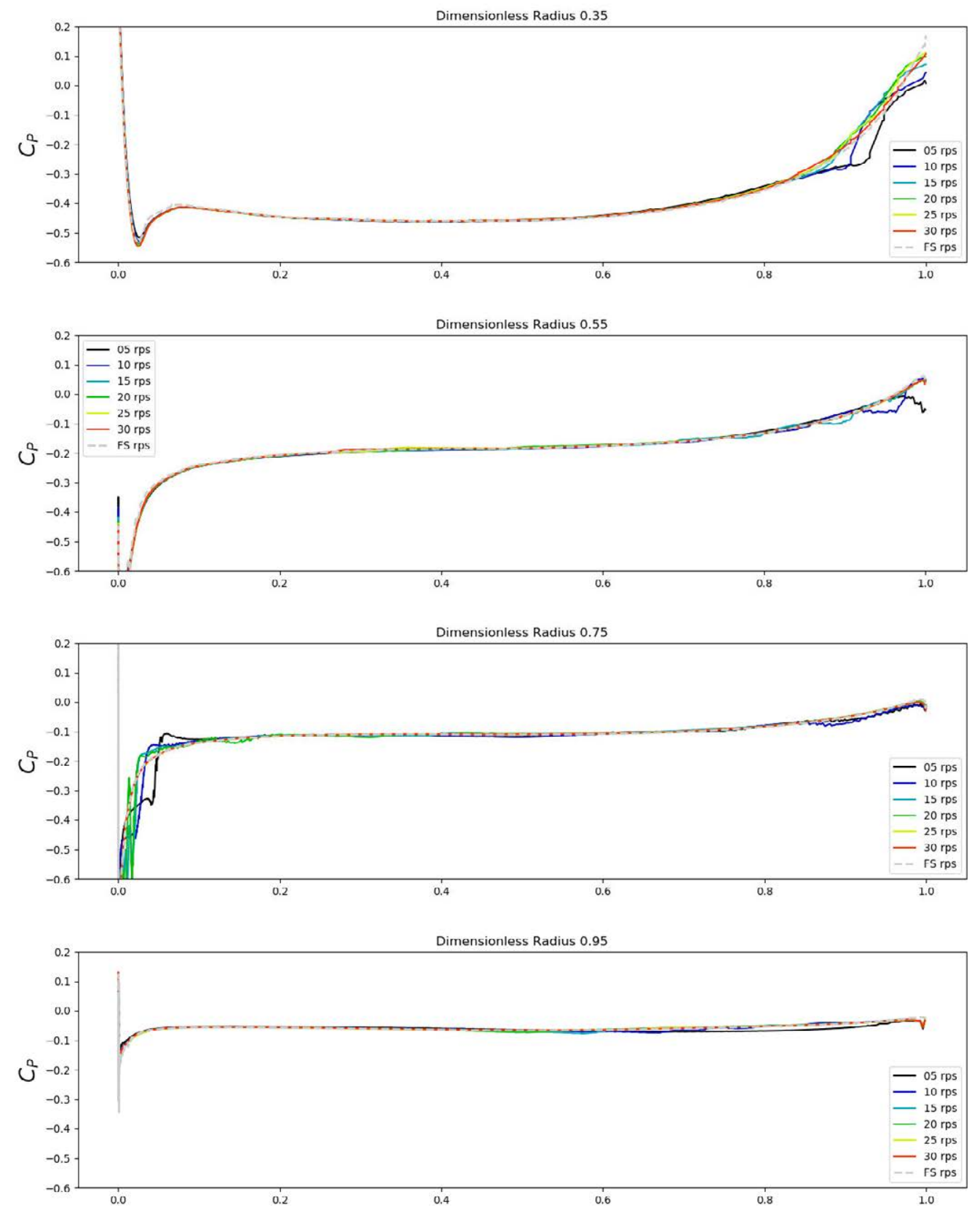

Figure B.12: Suction side of the $C_{p}$, for different radii of the CONV04 propeller. 

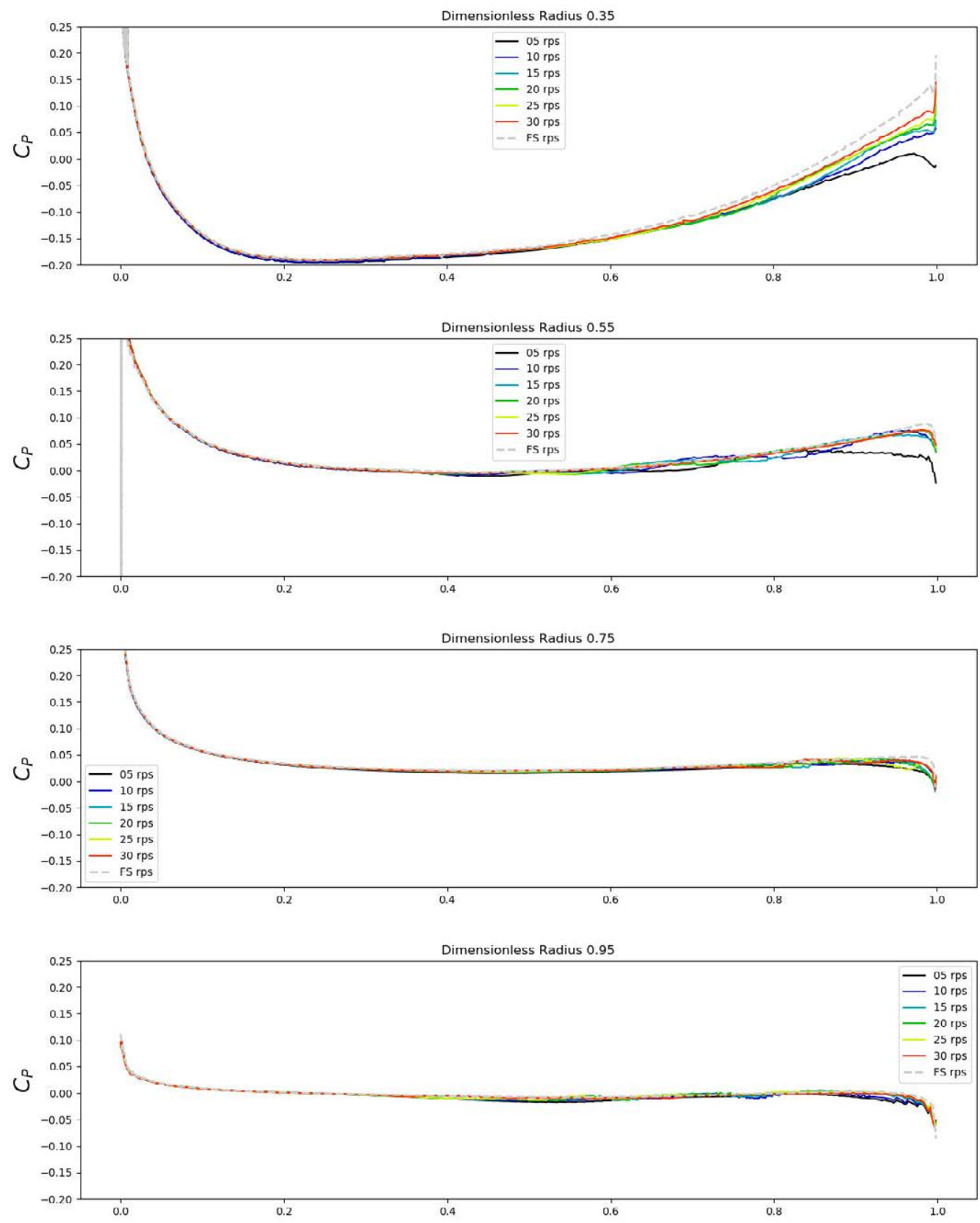

Figure B.13: Pressure side of the $C_{p}$, for different radii of the CONV04 propeller. 

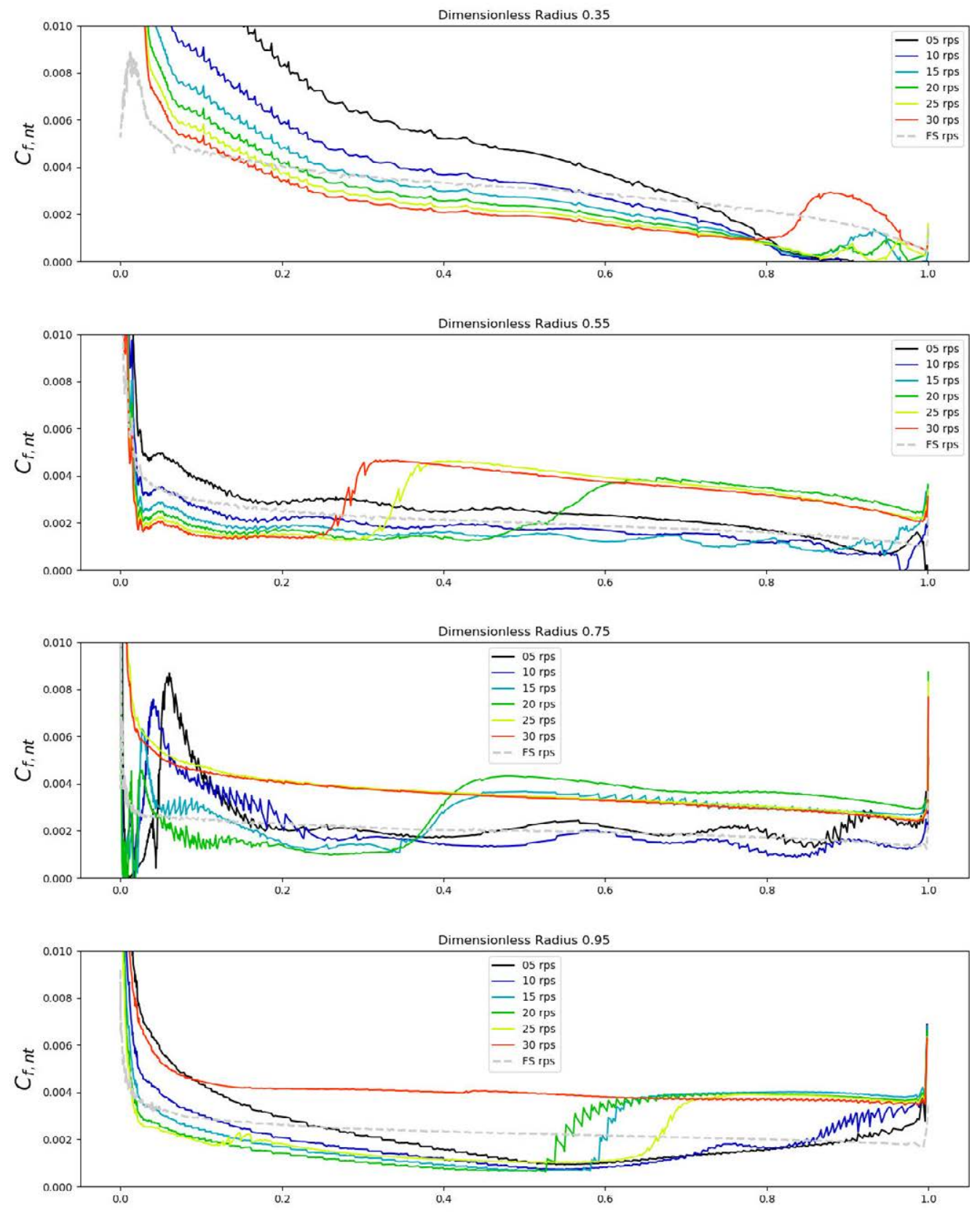

Figure B.14: Suction side of the $C_{f, n t}$, for different radii of the CONV04 propeller. 

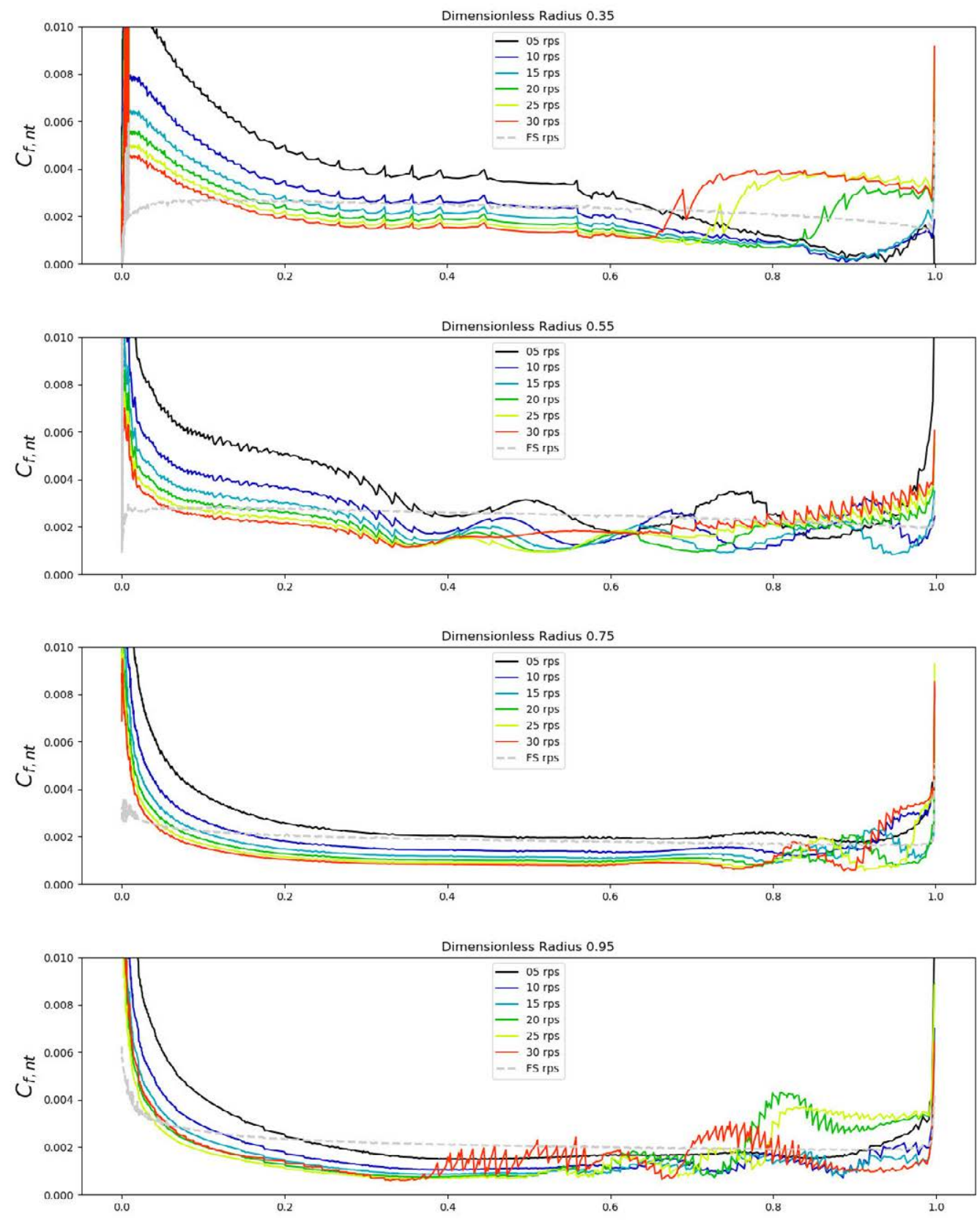

Figure B.15: Pressure side of the $C_{f, n t}$, for different radii of the CONV04 propeller. 
Mesh convergence for section 3.2. 
One of the most important things in order to trust the numerical computations is the mesh convergence process. In this section, the mesh convergence process is presented for each propeller used at section 3.2. The thrust $K_{t}$ and torque $K_{q}$ dimensionless coefficients are used as convergence parameters. For the rest of the propellers simulated in this Thesis, the meshes are similar to those presented in this appendix.

The computational domain has been designed taking into account the geometrical periodicity of the propeller in the azimuthal direction. The diameter of the propeller $D$ will be used as the characteristic length for the definition of the computational domain. The cylindrical sector has a length of $9 D$ and radius of $10 D$, inside of which the propeller plane will be placed at $3 D$ behind the inflow boundary and at $6 D$ ahead of the outflow boundary.

For the meshing strategy, several subdomains are used to control the mesh size. The mesh size of these domains is relative to the 'base size' $h^{s}$, which is one of the parameters changed during our mesh convergence process. Consequently, all subdomains are remeshed proportionally, that is what is called 'global mesh refinement'.

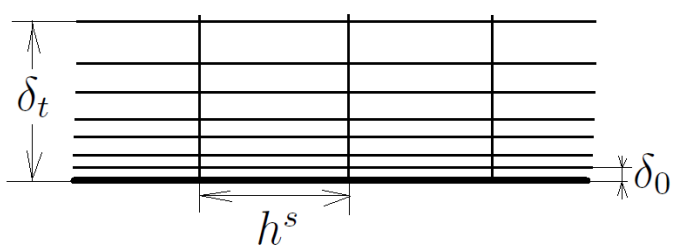

Figure C.1: Scheme of the parameters used for the convergence process in the boundary layer mesh.

The second parameter changed during the mesh convergence process is the number of prism layers in the boundary layer region, $N_{\text {Layers }}$, this number is varied between five and thirty, see fig (C.2). The thickness of each layer follows a geometric progression and the total thickness of the prism layer, $\delta_{t} \sim 200 \delta_{0}$, has been fixed for each propeller during the mesh convergence process. The mesh thickness near the wall, $\delta_{0}$, is set to the value that gives a $y^{+}<1$ all over the blade. This condition must be satisfied when the transition model is used.

A complete mesh convergence process was done for a different number of layers, $N_{\text {layers }}$, in the prism layer. In figure (C.2) it is possible to appreciate the different subdomains and a detailed view of the prism layer around the blade. The mesh convergence process was monitored for a different number of prism layers, and optimizes the most efficient value of prism layers to use inside the boundary layer. 


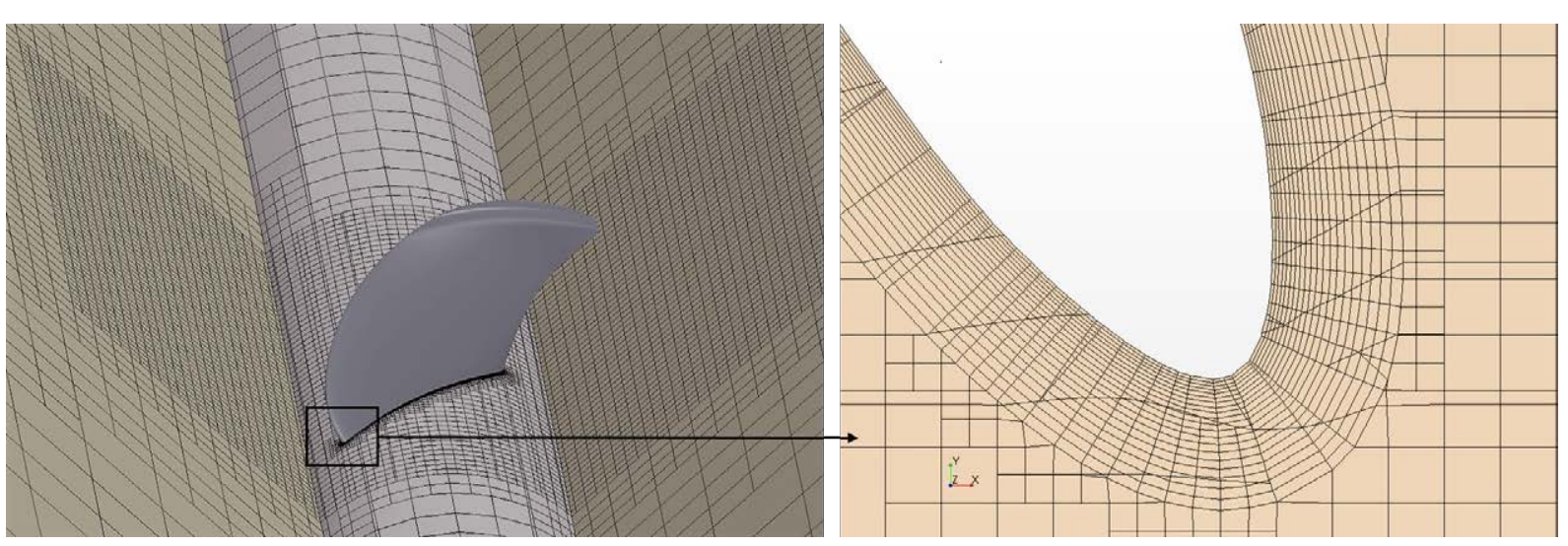

Figure C.2: Left: Mesh view close to the propeller. Right: Detailed view of the mesh in the leading edge proximities.

Results of the ITTC Benchmark Test Case for the well known VP1304 propeller in terms of dimensionless thrust and torque coefficients are presented in figure (C.3). Figure (C.3) shows the double convergence process, a first one decreasing the global base size for a constant number of layers in the boundary layer region, and a second pseudo-convergence process increasing the number of prisms layers. The result of this bi-parametric process, makes the converged value more trustworthy than other simpler mesh convergence processes. There is an additional advantage when this kind of studies is performed in order to save computational resources observing how the mesh parameters affect the final converged result. In figure (C.3), the dotted grey lines represent the convergence process when the global mesh size is fixed and the number of prisms layers is increased. It should be remarked that the number of layers in the boundary layer area seems to be a more relevant choice in the mesh design than the global mesh size. Consequently, meshes with more than 20 prism layers will always have an accurate approximation to the converged final values of $K_{t}$ and $10 K_{q}$ even when the total number of cells is less than just a third of the finest mesh.

Analogously in figure (C.4), the mesh convergence study for a P1727 Tip-Rake propeller based on the global mesh size and the number of prisms layers in the boundary layer region is presented. It can be observed in figure (C.4) that with in this kind of propeller, when the number of cells is over a critical number, around 10 million in this case, the converged values are accurately computed even if the number of prism layers is not excessive.

Finally, figure (C.5) shows the mesh convergence study of the new generation of $C L T$ propellers in terms of dimensionless thrust and torque coefficients. 


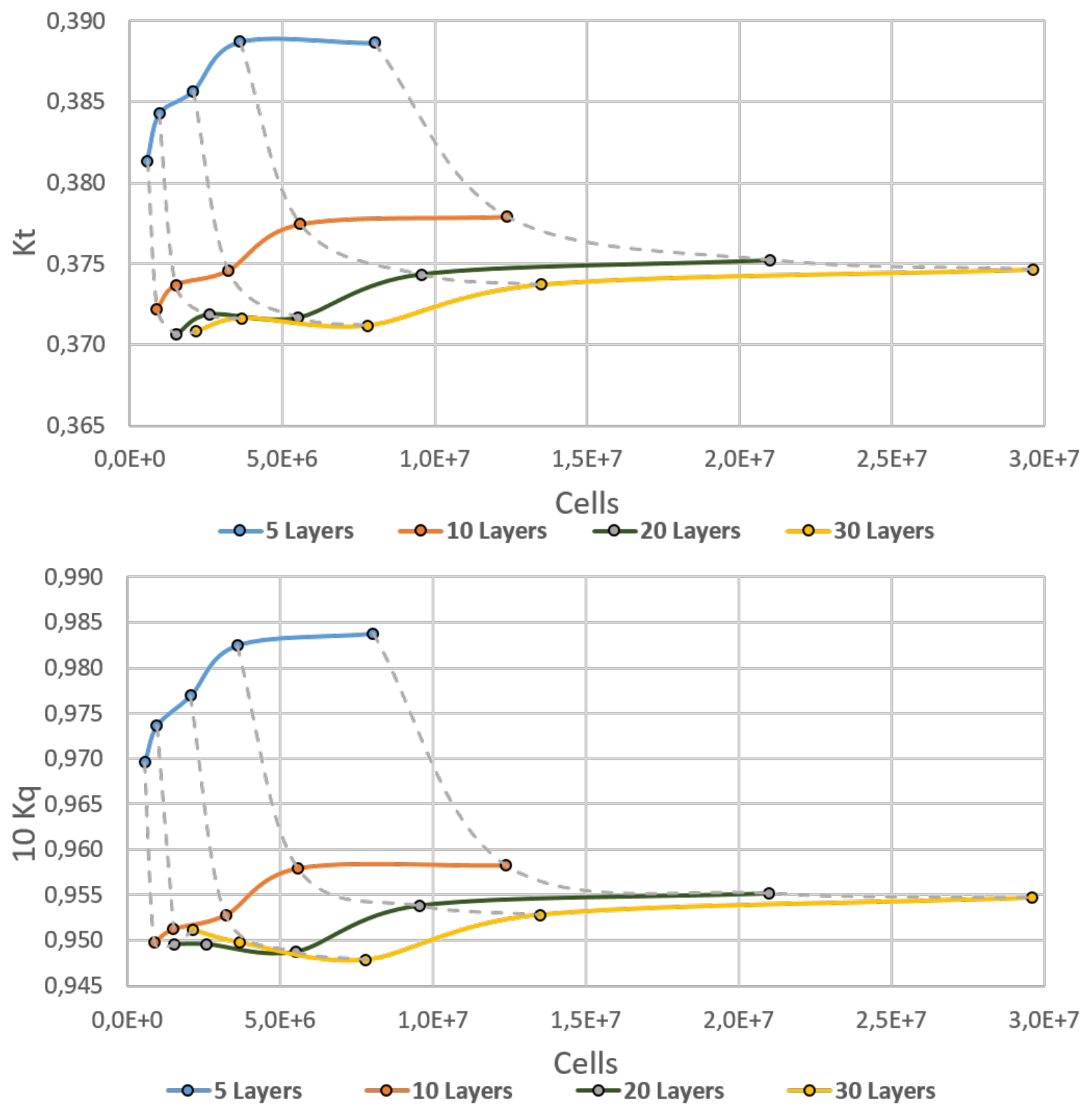

Figure C.3: Mesh convergence process for VP1304 propeller based on the $K t$ and $10 K q$ non-dimensional parameters for different number of prism layers. Dotted grey lines represent the convergence process when the global mesh size is fixed and the number of prism layers is increased.

After this mesh convergence process performed for these three types of propellers, we can assume that the errors in the future calculations due to the mesh design or mesh sizes will be reasonably controlled, and the significant differences when compared to the reference values will be more related to the modelization of the complex physics that appears in these problems. 

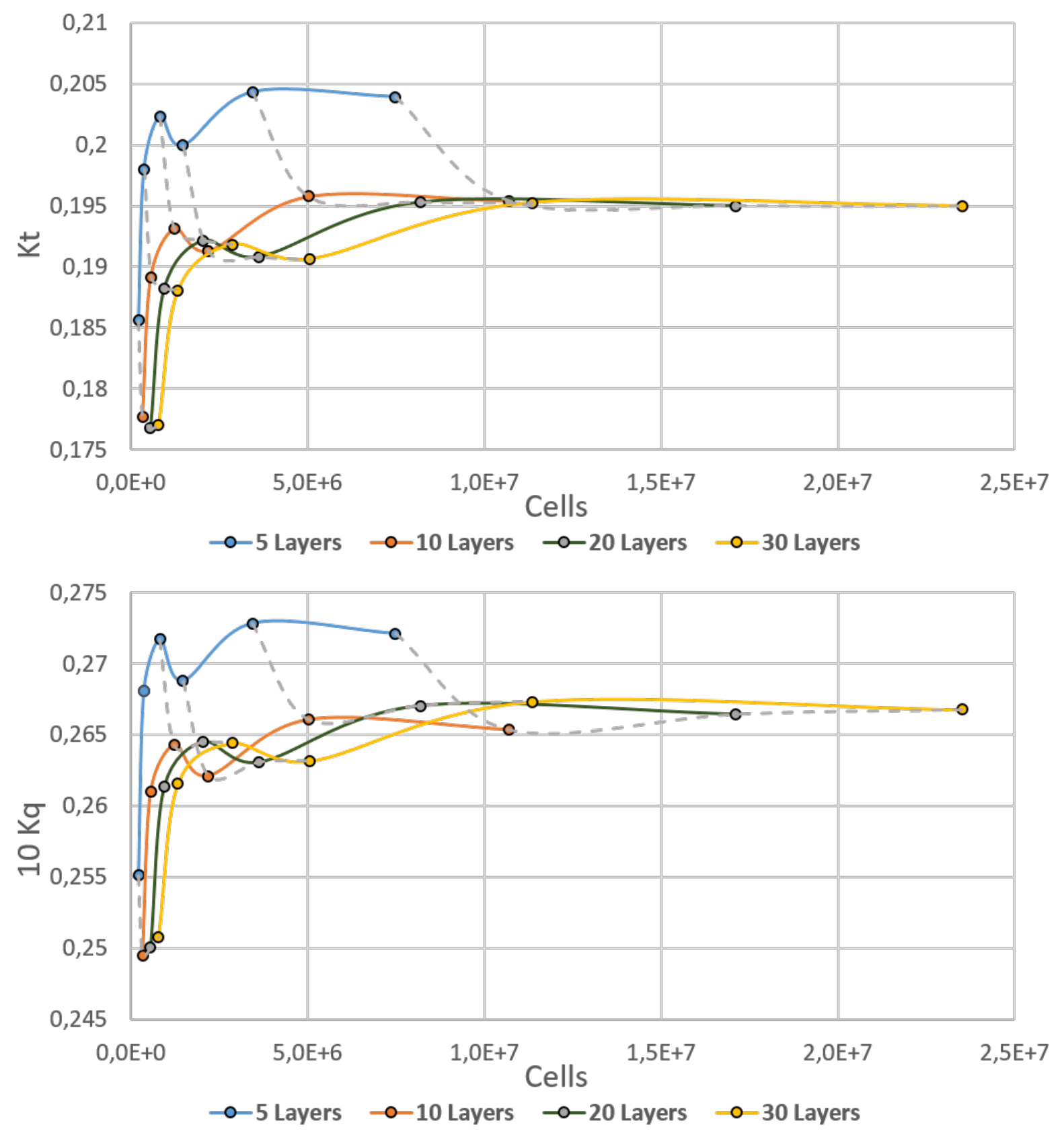

Figure C.4: Mesh Convergence process for the P1727 propeller based on the $\mathrm{Kt}$ and $10 \mathrm{Kq}$ non-dimensional parameters for different number of prism layers. Dotted grey lines represent the convergence process when the global mesh size is fixed and the number of prism layers is increased. 

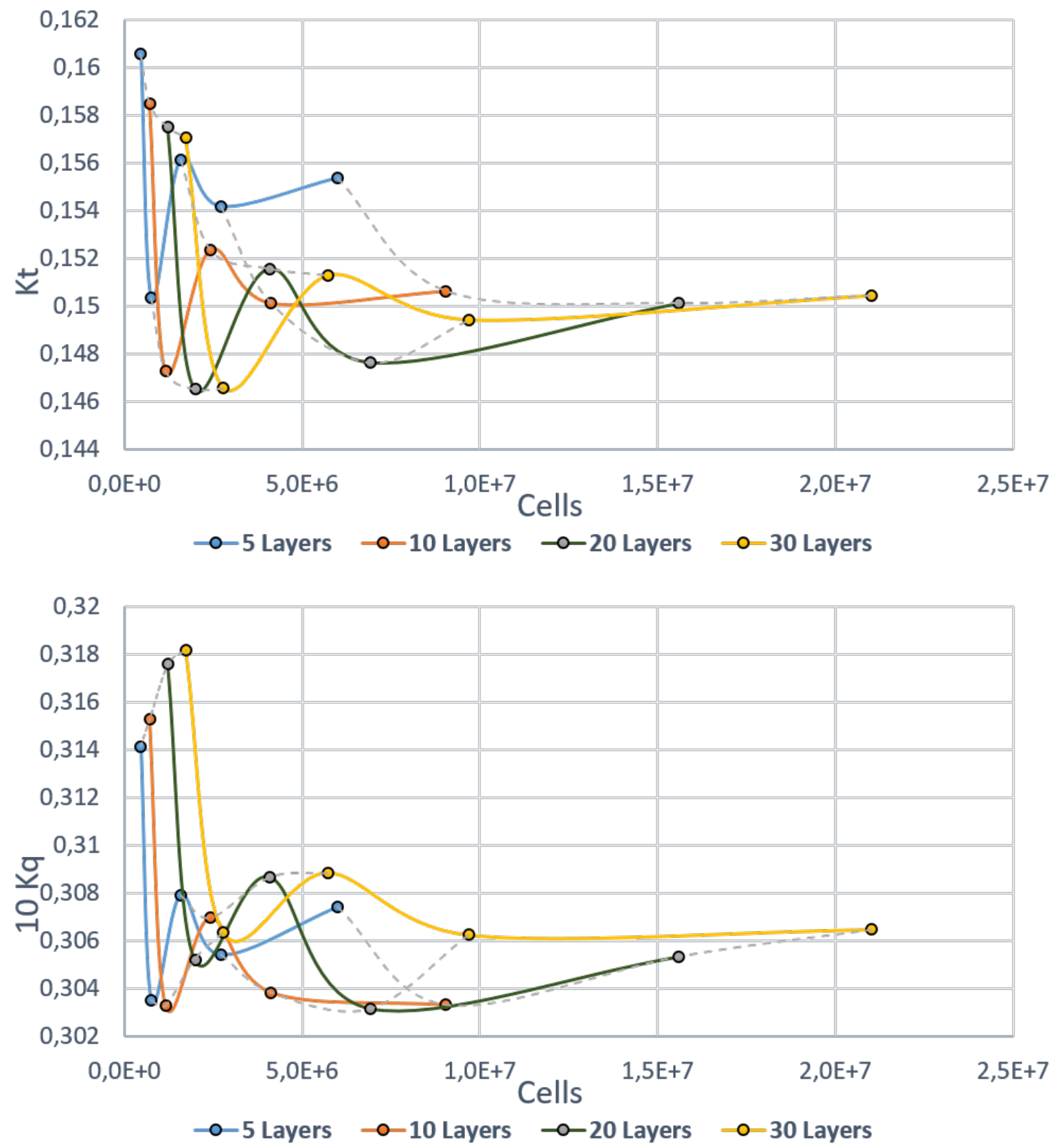

Figure C.5: Mesh Convergence process for different numbers of layers in the boundary layer for CLT propeller. Kt and $\mathrm{Kq}$ values. 


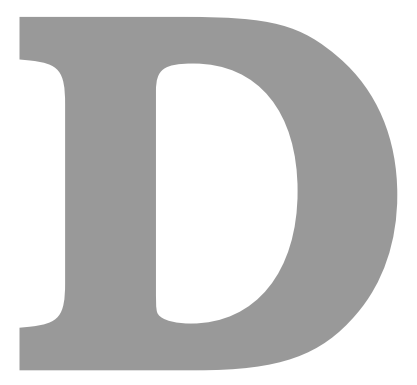

\section{Detailed description of the new} friction coefficient estimation methods 


\section{D.1 Modified STRIPSIS method.}

This method is a modification of the method presented by Perez-Sobrino et al. (2016). The original method is a strip-based extrapolation method and the main difference with the $I T T C-78$ and the $S V A$ methods is the consideration of different flow areas in the propeller blade. The Reynolds ranges for the different types of flow are given by the Equation (D.1, D.2) which depend on the chord $(\mathrm{Cr})$ and roughness $\left(k_{p}\right)^{1}$.

$$
\begin{gathered}
R e_{c, L}^{o}=90 \frac{C r}{k_{p}} \\
R e_{c, T}^{o}=415 \frac{C r}{k_{p}}
\end{gathered}
$$

Depending if the sections are considered as laminar or turbulent, the friction coefficients are calculated with the Blasius or the Prandtl-Von Karman friction line respectively. For those in the transition zone an Reynolds-based interpolation between the friction coefficient values corresponding to laminar and turbulent limits. The rest of the procedure is well explained at Perez-Sobrino et al. (2016).

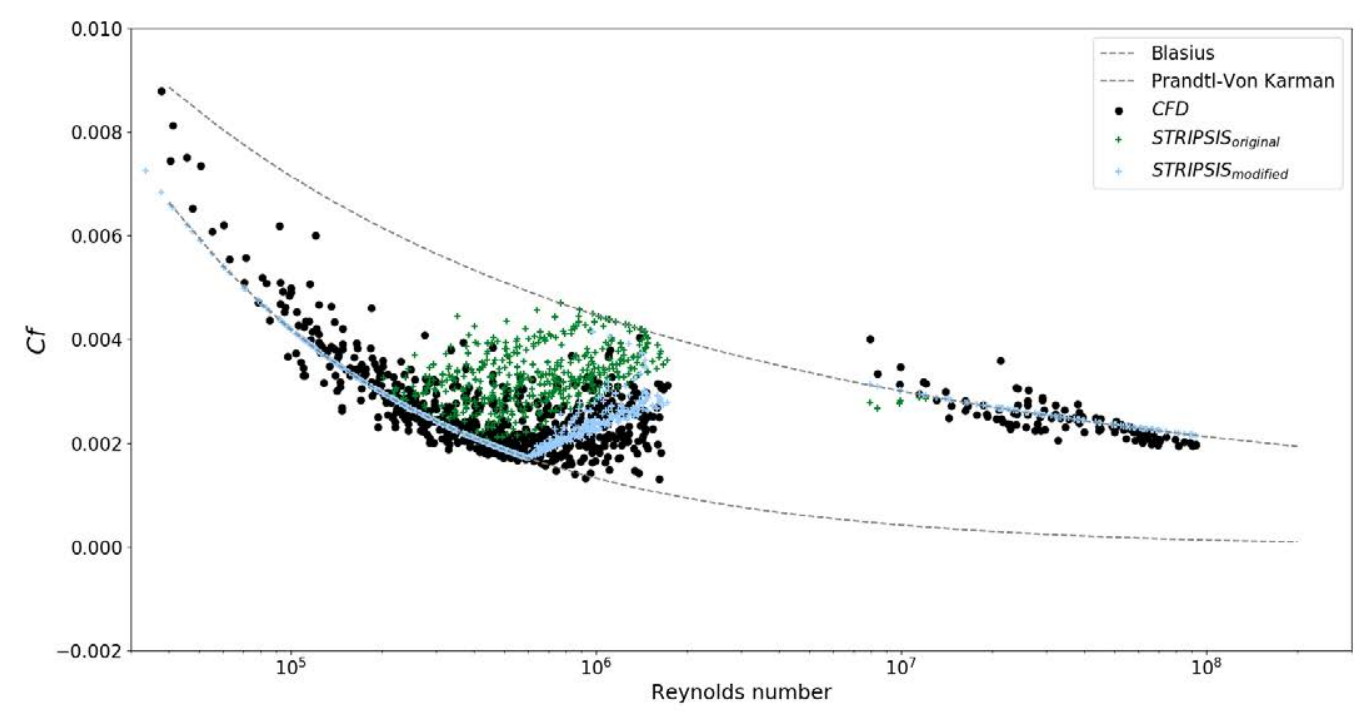

Figure D.1: Estimation of the friction coefficients for the Original STRIPSIS and modified STRIPSIS methods for differents sections of the blade.

In the modification of the method the objective is to tune the critical Reynolds numbers $\left(R e_{c r i t}\right)$ at model and at full scale to improve the results compared with CFD simulations. Results obtained with the new parameters proposed in equations D. 3 and D.4, are presented in figure D.1. The critical Reynolds

\footnotetext{
${ }^{1} k_{p}$ is considered $20 e-6 \mathrm{~m}$ for model and $30 e-6 \mathrm{~m}$ for full scale.
} 
number for laminar flow $\operatorname{Re}_{c, L}$ at model scale has been fixed to $6.0 \cdot 10^{5}$ to match the behavior of the inflection point in the CFD results. With respect to the critical Reynolds number for turbulent flow $R e_{c, T}$ at model scale, the cofficient 450 have changed to 350 to maintain the slope of the transition flow area.

$$
\begin{array}{r}
R e_{c ., L}^{m}=6.0 e 5 \\
R e_{c ., T}^{m}=350 * \frac{C r}{k_{p}}
\end{array}
$$

As full scale the flow over the propeller blade has been assume as turbulent. This consideration is basically due to the intrinsic turbulence of the incoming flow when the propeller is working at the ship wake.

\section{D.2 Multilayer Perceptron Friction coefficient estimator.}

For the creation of the Multilayer Perceptron that estimates the friction coefficient of each section of the propeller, different types of configuration and input parameters have been studied.

The are many variables involved in this problem some related with the flow conditions such as the Reynolds, others related with the geometry of the section such as thickness-chord ratio, and others relative to the radial position of the section studied, etc... With the aim to develop a new model based on neural networks, being capable of take into account some of those parameters and to reproduce the fig 3.23 , a comparative analysis has been performed in order to choose the most accurate configuration of the MLP for the particular dataset of propellers presented in appendix $B$.

There are different aspects that could be changed in the definition of a multilayer perceptron:

- Input parameters:

The input parameters of the MLP should be those variables that are important in the problem to model. In this case the author considers important the following variables:

$$
\begin{aligned}
& \text { - Re } \\
& \text { - THCR } \\
& \text { - FCR } \\
& \text { - X0 } \\
& \text { - Profile type (PT). }
\end{aligned}
$$


Due to the limited dataset, the MLP only takes as an input parameters the Reynolds number, the thickness-chord ratio and the camber-chord ratio.

\section{- Number of hidden layers.}

The number of hidden layers represents the level of abstraction of the neural network. A variation have been set to a maximum of two layers due to the number size of the database used to train the network.

\section{- Number of neurons of each hidden layer.}

This and the previous are the most difficult elections during the design process. In order to find the optimum for this particular case, those two parameters has been varied.

- Activation functions (Linear, Sigmoid, ELU, ReLu, etc...)

For regression analysis are commonly used, the ReLu at the hidden layers and the Linear activation function for the output layer. As a starting point this configuration has been used. The sigmoid activation function is no longer used since the ReLu was presented.

- Training/Testing dataset The training/testing dataset is the group of data that is going to be used to train and test the neural network. In other words, it is the database that is going to be use to generate the model of the problem.

A conceptual overview of the MLP and is presented in figure (D.2). The number of hidden layers have been change between 1 and 2 with a combination of neurons between 1 and 20 for the first hidden layer and between 1 and 10 for the second hidden layer.

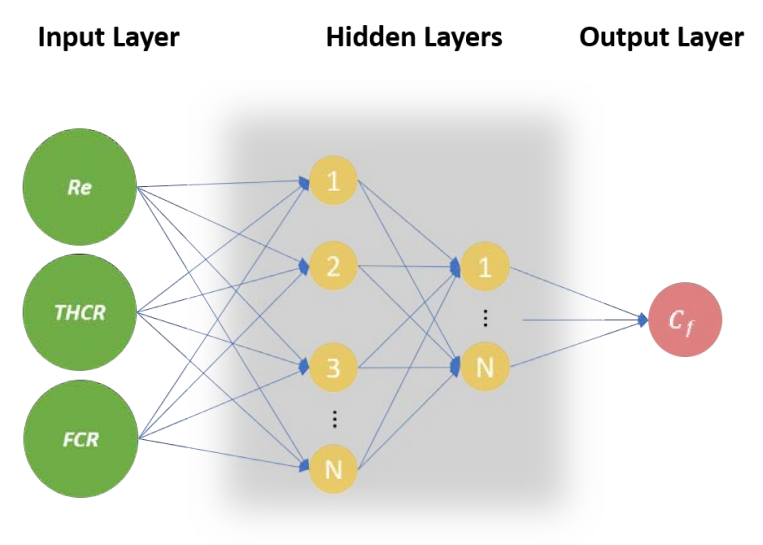

Figure D.2: Base $A N N$ scheme.

Results for all the cases studied is presented in D.3, the optimum Neural Network (NN) after this study is the configuration with 20 neurons in the first hidden layer and 9 neurons in the second hidden 


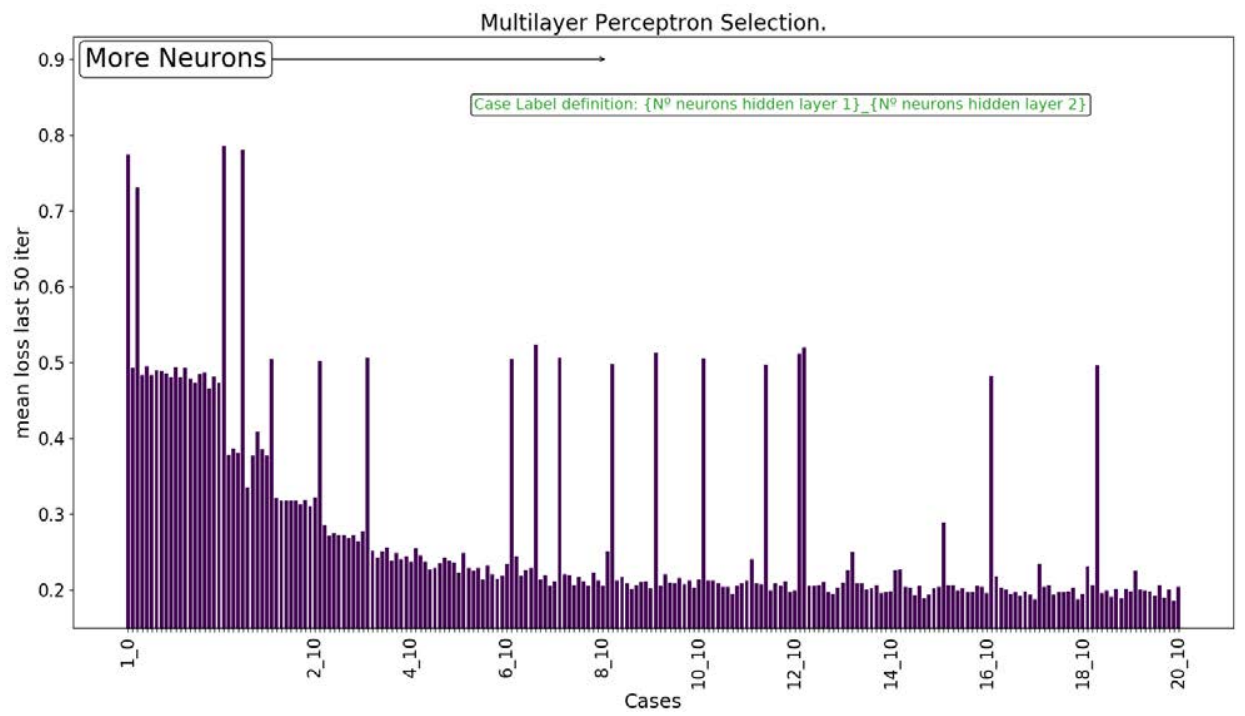

Figure D.3: Mean loss of the 20 last iteration of the different cases studied for select the optimum one $M L P$ configuration.

layer. A comparison with a MLP of 3 neurons in the first hidden layer and 2 in the second in presented at figure D.4. A more detailed analysis of the final configuration have been done changing the activation functions for the hidden layers between the traditional sigmoid, and the moderns ReLu and ELU. Results shown that the best option in terms of accuracy and speed of convergence is the exponential linear unit 'ELU' activation function and is presented in figure D.5.

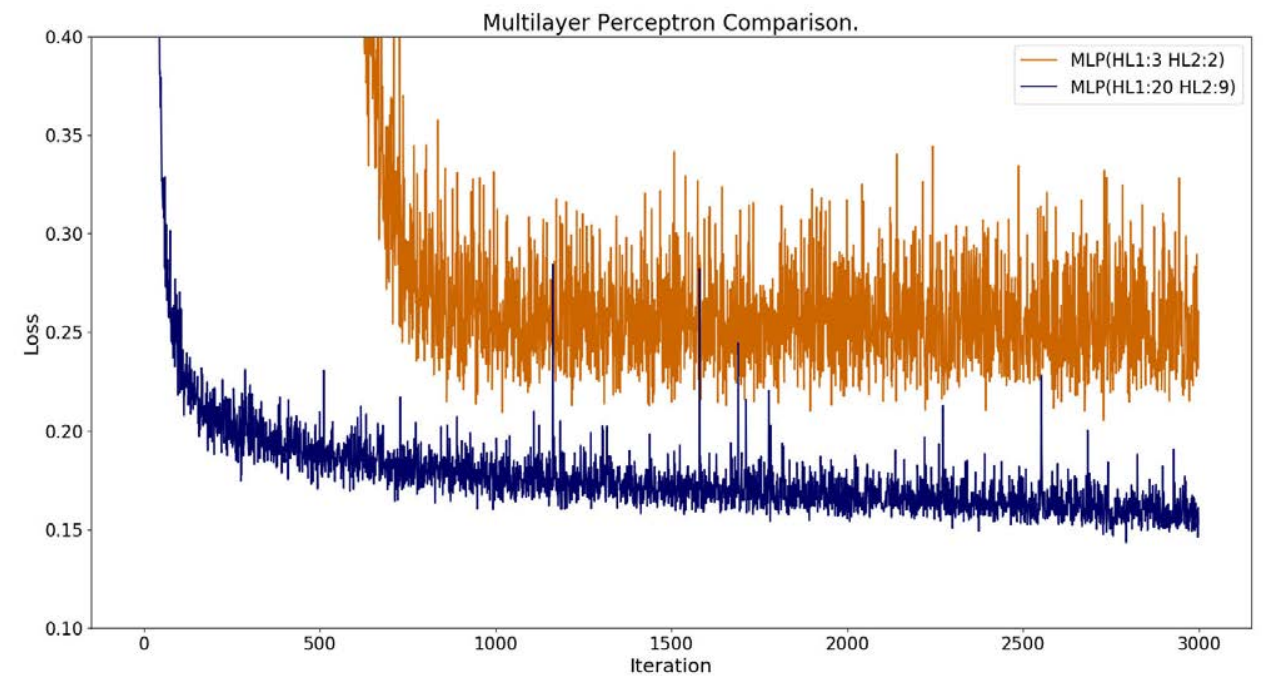

Figure D.4: Residuals comparison of the final $M L P$ configuration and one of the alternatives studied. 


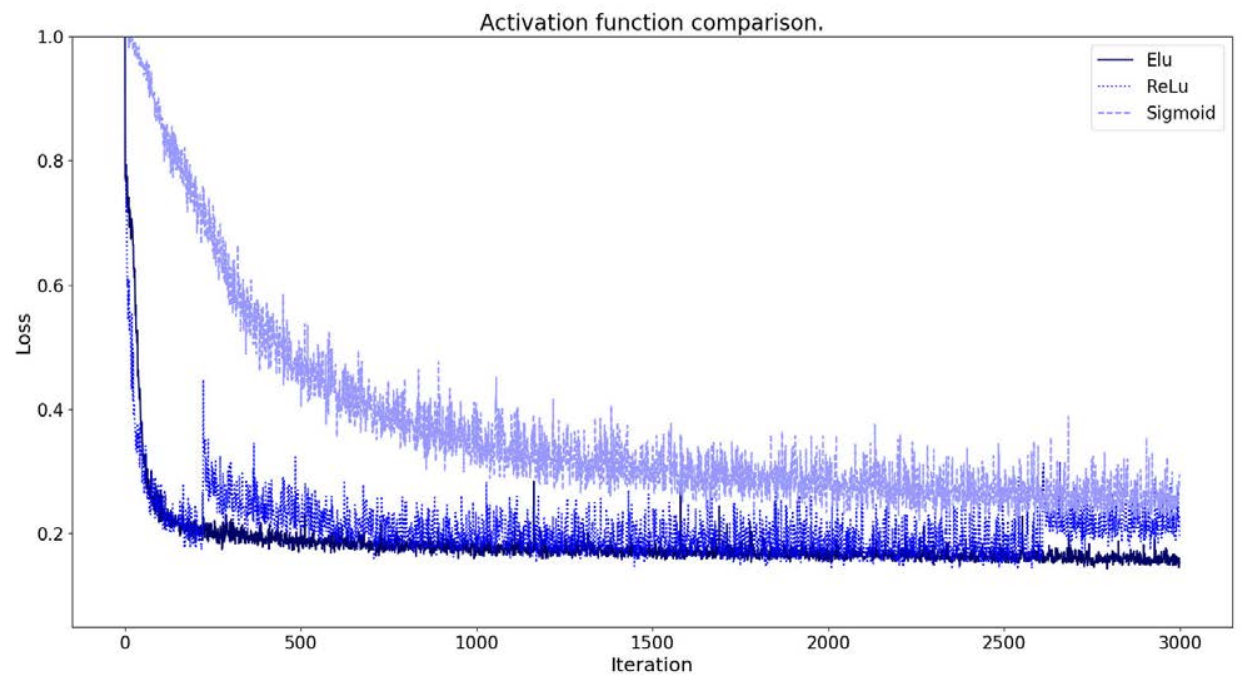

Figure D.5: Residuals comparison for different activation function of the hidden layers. 